\title{
Development of Scientific Simulation 3D Full wave ICRF code for Stellarators and Heating/CD scenarios development
}

\author{
Vdovin V.L. \\ Russian Research Centre "Kurchatov Institute" \\ Work supported by the U.S. Department of Energy \\ under Grant DE-FG02-02ER54667
}

\begin{abstract}
In this report we describe theory and $3 \mathrm{D}$ full wave code description for the wave excitation, propagation and absorption in 3-dimensional (3D) stellarator equilibrium high beta plasma in ion cyclotron frequency range (ICRF). This theory forms a basis for a 3D code creation, urgently needed for the ICRF heating scenarios development for the operated LHD [1], constructed W7-X [2], NCSX [3] and projected CSX3 stellarators, as well for re evaluation of ICRF scenarios in operated tokamaks and in the ITER. The theory solves the 3D Maxwell-Vlasov antenna-plasma-conducting shell boundary value problem in the non - orthogonal flux coordinates $(\psi, \theta, \varphi), \psi$ being magnetic flux function, $\theta$ and $\varphi$ being the poloidal and toroidal angles, respectively. All basic physics, like wave refraction, reflection and diffraction are self consistently included, along with the fundamental ion and ion minority cyclotron resonances, two ion hybrid resonance, electron Landau and TTMP absorption. Antenna reactive impedance and loading resistance are also calculated and urgently needed for an antenna -generator matching. This is accomplished in a real confining magnetic field being varying in a plasma major radius direction, in toroidal and poloidal directions, through making use of the hot dense plasma wave induced currents with account to the finite Larmor radius effects.

We expand the solution in Fourier series over the toroidal $(\varphi)$ and poloidal $(\theta)$ angles and solve resulting ordinary differential equations in a radial like $\psi$ - coordinate by finite difference method. The constructed discretization scheme is divergent - free one, thus retaining the basic properties of original equations. The Fourier expansion over the angle coordinates has given to us the possibility to correctly construct the "parallel"

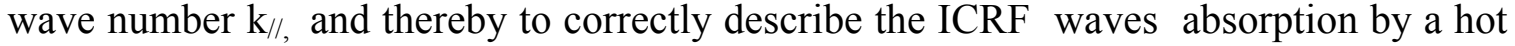
plasma.

The toroidal harmonics are tightly coupled with each other due to magnetic field inhomogenity of stellarators in toroidal direction. This is drastically different from axial symmetric plasma of the tokamaks. The inclusion in the problem major radius variation of magnetic field can strongly modify earlier results obtained for the straight helical, especially for high beta plasma, due to location modification of the two ion hybrid resonance layers. For the NCSX, LHD, W7-AS and W7-X like magnetic field topology inclusion in our theory of a major radius inhomogenity of the magnetic field is a key element for correct description of RF power deposition profiles at all.

The theory is developed in a manner that includes tokamaks and magnetic mirrors as the particular cases through general metric tensor (provided by an equilibrium solver) treatment of the wave equations.
\end{abstract}


We describe that newly developed stellarator ICRF 3D full wave code PSTELION, based on theory described in this report. Application to tokamaks, ITER, stellarators and benchmarking with 2D TORIC and 3D AORSA codes is given in attached subreports $[15,16,17]$.

\section{Introduction}

\section{Full Wave analysis in stellarators}

The important initial step to the problem solution has been done in [4]. Here we are continuing the investigations on ICRF plasma heating and current drive.

Motivation

Mainly ICRF heating (and possibly Current Drive) in W7 -AS, W7-X and other machines (scenarios development, optimised antennae, etc.)

Also

- Low frequency instabilities (Alfven eigenmodes)

- Diagnostics (Ion Cyclotron Emission)

- Features

- Configuration: 3D, 2D (Axis - symmetric)

- Plasma model: Hot plasma ( Kinetic Alfven Waves included; FLR ions and electrons effects )

- Numerical method:

Finite difference, Modes Expansion

\section{Magnetic flux coordinates}

Glux coordinates (non orthogonal)

- minor radius direction : poloidal magnetic flux $\psi$

- poloidal direction: angle $\theta$ 
- toroidal direction: angle $\varphi$

- contravariant basis is

$$
e^{1}=\nabla \psi, \quad e^{2}=\nabla \theta, \quad e^{3}=\nabla \varphi
$$

口 J: Jacobian

$$
J=\frac{1}{\nabla x^{1}\left[\nabla x^{2} x \nabla x^{3}\right]}=\frac{1}{\nabla \psi[\nabla \theta x \nabla \varphi]}
$$

g: Metric tensor $g_{i j}=\hat{e}_{i} \hat{e}_{j}$, where covariant basis vectors are

$$
\widehat{e}_{i} \equiv \partial \vec{r} / \partial x^{i}
$$

\section{FULL WAVE EQUATION}

This report develops a 3D full wave calculation, which uses poloidal and toroidal harmonic expansions to solve the wave equation $\left(\mathrm{k}_{0}=\omega / \mathrm{c}, \mathrm{c}-\right.$ speed of light):

$$
\operatorname{rotrot} \vec{E}=\frac{4 \pi i k_{0}}{c}\left(\vec{j}_{p}+\vec{j}^{e x t}\right)+k_{0}^{2} \vec{E}
$$

in flux coordinates with appropriate boundary conditions, in 3D stellarator geometry. In eq.(1.1) $\mathbf{j}^{\mathbf{e x t}}$ is an imposed antenna current density and $\mathbf{j}_{\mathbf{p}}$ is the RF plasma current, which is evaluated by solution of the linearized Vlasov equation and in principal is an integral functional of the wave electric field $\boldsymbol{E}$. In the ion cyclotron frequency range it is frequently justified to make use the small Larmor radius approximation for $\mathbf{j}_{\mathbf{p}}$

$$
J_{p}=J^{(0)}+J^{(2)}
$$

where upper suffixes denote the order in the finite Larmor radius (FLR) expansion. Here we followed to procedure starting from the results of [5], in which the Vlasov equation is solved in plane-stratified geometry, and assuming that the vector form of $\mathbf{j}_{\mathbf{p}}$ in this limit holds also in 3D toroidal geometry. This approach was fruitfully used in the TORIC 2D code [6] and recently have been confirmed by AORSA code in comparison ICRF modellings for C-mod tokamak [7].

Code also contains the option frequently named as "as reduced order" approach (ORA), 
which is significantly smaller computer memory and time consuming, when $\mathbf{j}_{\mathbf{p}}$ is determined by the relation $\vec{j}_{p}=\vec{\sigma} \vec{E}$. with $\vec{\sigma}=\vec{\sigma}^{(0)}+i k_{\perp} \vec{\sigma}^{(1)}-k_{\perp}^{2} \vec{\sigma}^{(2)}$ to "correct" zero Larmor radius ion current by adding appropriate FLR corrections to the dielectric hot plasma tensor. The $k_{\perp}$ is the perpendicular wave vector of the Fast Wave, derived by solving local WKB dispersion relation with FLR corrections. In that ORA option we rewright the eq.(1.1) more compactly through the plasma dielectric tensor [8] $\vec{\varepsilon}$ as

$$
\operatorname{rotrot} \vec{E}=k_{0}^{2} \vec{\varepsilon} \vec{E}+\frac{4 \pi i k_{0}}{c} \vec{j}^{e x t}
$$

It means that ORA simply installs in finite ion Larmor radius expansion terms, proportional to the $\left(\mathrm{k}_{\perp} \rho_{\mathrm{i}}\right)^{2}$, the Alfvenian perpendicular wave number. That procedure rightly describes the absorbed RF power coming to the Bernstein waves [ 4 ]. At low phase velocities $\omega / k_{\mathbf{I}}<v_{T e}$ the finite Larmor radius corrections are insignificant ones and Fast Waves are converted to the kinetic Alfven waves (KAW) at $\omega<\omega_{\text {ci }}$ and this will be correctly described by our ORA hot kinetic tensor description.

\section{Formulation}

To express the plasma wave induced currents in simplest form, we write the electrical field orthogonal components in local co-ordinates as $E_{\psi}, E_{N}, E_{b}$, where b-component is parallel to the total confining magnetic field $\mathrm{B}_{0}$.

We choose now to work in the orthogonal set of unit vectors

$$
\hat{e}_{\psi}=\frac{\nabla \psi}{|\nabla \psi|}, \quad \hat{e}_{b}=\frac{\vec{B}_{0}}{\left|B_{0}\right|}, \quad \hat{e}_{N}=\widehat{e}_{b} \times \widehat{e}_{\psi}
$$

Any wave vector a is expanded over these vectors as

$$
\vec{a}=a_{1} \hat{e}_{\psi}+a_{2} \hat{e}_{N}+a_{3} \hat{e}_{b}
$$

The needed ROT (or curl) operators are easily calculated in covariant basis vectors $\hat{e}_{j}$ and useful is to expand them over new orthogonal basis:

$$
\hat{e}_{1}=\xi_{11} \hat{e}_{\psi}+\xi_{12} \hat{e}_{N}+\xi_{13} \hat{e}_{b}
$$




$$
\begin{aligned}
& \hat{e}_{2}=\xi_{21} \hat{e}_{\psi}+\xi_{22} \hat{e}_{N}+\xi_{23} \hat{e}_{b} \\
& \hat{e}_{3}=\xi_{31} \hat{e}_{\psi}+\xi_{32} \hat{e}_{N}+\xi_{33} \hat{e}_{b}
\end{aligned}
$$

It is straightforward to obtain:

$$
\begin{aligned}
& \xi_{11}=\frac{1}{|\nabla \psi|} \\
& \xi_{12}=\frac{1}{|\nabla \psi| J}\left\{c_{3}\left(g_{12} g_{33}-g_{13} g_{23}\right)+c_{2}\left(g_{12} g_{23}-g_{13} g_{22}\right)\right\} \\
& \xi_{13}=c_{2} g_{12}+c_{3} g_{13} \\
& \xi_{21}=0, \quad \xi_{31}=0 \\
& \xi_{22}=|\nabla \psi| J c_{3} \\
& \xi_{32}=-c_{2}\left(g_{22} g_{33}-g_{23}^{2}\right)^{1 / 2}, \quad \xi_{33}=c_{3} g_{33}+c_{2} g_{23} \\
& \xi_{23}=c_{2} g_{22}+c_{3} g_{23}
\end{aligned}
$$

\section{Differential operators in 3D non orthogonal coordinates}

\subsection{Curl operators}

In terms of these unit vectors the curl operators take the form in psi direction:

$$
\left.\operatorname{rot} \vec{a}\right|_{\psi}=\frac{1}{|\nabla \psi| J}\left\{\frac{\partial}{\partial \theta}\left[a_{3}\left(c_{3} g_{33}+c_{2} g_{23}\right)-c_{2} \gamma a_{2}\right]-\frac{\partial}{\partial \varphi}\left[c_{3} \gamma a_{2}+a_{3}\left(c_{2} g_{22}+c_{3} g_{23}\right)\right]\right\}
$$

or

$$
\left.\operatorname{rot} \vec{a}\right|_{\psi}=\varsigma_{11} \frac{\partial a_{3}}{\partial \theta}+\varsigma_{12} \frac{\partial a_{2}}{\partial \theta}+\varsigma_{13} \frac{\partial a_{3}}{\partial \varphi}+\varsigma_{14} \frac{\partial a_{2}}{\partial \varphi}+\gamma_{12} a_{2}+\gamma_{13} a_{3}
$$

where 


$$
\begin{array}{ll}
\varsigma_{11}=\frac{1}{|\nabla \psi| J}\left(c_{3} g_{33}+c_{2} g_{23}\right), & \varsigma_{12}=-c_{2}, \\
\varsigma_{13}=-\frac{1}{|\nabla \psi| J}\left(c_{2} g_{22}+c_{3} g_{23}\right), & \varsigma_{14}=-c_{3}
\end{array}
$$

and

$$
\begin{aligned}
& \gamma_{12}=-\frac{1}{|\nabla \psi| J}\left[\frac{\partial}{\partial \theta}\left(c_{2} \gamma\right)+\frac{\partial}{\partial \varphi}\left(c_{3} \gamma\right)\right] \\
& \gamma_{13}=\frac{1}{|\nabla \psi| J}\left[\frac{\partial}{\partial \theta}\left(c_{3} g_{33}+c_{2} g_{23}\right)-\frac{\partial}{\partial \varphi}\left(c_{2} g_{22}+c_{3} g_{23}\right)\right] \\
& \gamma=\left(g_{22} g_{33}-g_{23}^{2}\right)^{1 / 2}
\end{aligned}
$$

Binormal (in $\mathrm{e}_{\mathrm{N}}$ direction) the curl component is

$$
\begin{aligned}
\left.\operatorname{rot} \vec{a}\right|_{N}= & \varsigma_{21} \frac{\partial a_{2}}{\partial \psi}+\varsigma_{22} \frac{\partial a_{3}}{\partial \psi}+\varsigma_{23} \frac{\partial a_{3}}{\partial \theta}+\varsigma_{24} \frac{\partial a_{1}}{\partial \theta}+\varsigma_{25} \frac{\partial a_{3}}{\partial \varphi}+\varsigma_{26} \frac{\partial a_{2}}{\partial \varphi}+\varsigma_{27} \frac{\partial a_{1}}{\partial \varphi}+ \\
& \gamma_{21} a_{1}+\gamma_{22} a_{2}+\gamma_{23} a_{3},
\end{aligned}
$$

where

$$
\begin{aligned}
& \varsigma_{21}=\frac{1}{J}\left(\xi_{22} c_{2} \gamma-\left|\xi_{32}\right| c_{3} \gamma\right)=0 \\
& \varsigma_{22}=-\frac{1}{J}\left[\left(c_{3} g_{33}+c_{2} g_{23}\right) \xi_{22}+\left|\xi_{32}\right|\left(c_{2} g_{22}+c_{3} g_{23}\right)\right]=-\frac{\gamma}{J} \\
& \varsigma_{23}=|\nabla \psi| \frac{g_{12} g_{33}-g_{13} g_{23}}{g_{22} g_{33}-g_{23}^{2}} \\
& \varsigma_{24}=c_{2}
\end{aligned}
$$




$$
\begin{aligned}
& \varsigma_{25}=\frac{\xi_{22}}{J}\left(c_{2} g_{12}+c_{3} g_{13}\right)-\frac{\xi_{12}}{J}\left(c_{2} g_{22}+c_{3} g_{23}\right)=\frac{1}{\gamma J}\left(g_{22} g_{13}-g_{12} g_{23}\right) \\
& \varsigma_{26}=\frac{\xi_{22}}{J} \beta-\frac{\xi_{12}}{J} c_{3} \gamma=0 \\
& \beta=\frac{1}{|\nabla \psi| J}\left[c_{3}\left(g_{12} g_{33}-g_{13} g_{23}\right)+c_{2}\left(g_{12} g_{23}-g_{13} g_{22}\right)\right] \\
& \varsigma_{27}=c_{3} \\
& \gamma_{21}=\frac{\xi_{22}}{J}(v)_{\varphi}^{\prime}+c_{2}|\nabla \psi|(v)_{\theta}^{\prime}
\end{aligned}
$$

Here and below designations $(\ldots)_{\theta}^{\prime}$, etc. mean partial derivatives over $\theta, \psi, \varphi$ like

$$
\begin{aligned}
& \frac{\partial}{\partial \theta}(\ldots), \text { etc. } \\
& \qquad \begin{array}{l}
|\nabla \psi| \\
\gamma_{22}=\frac{\xi_{22}}{J}\left[(\beta)_{\varphi}^{\prime}+\left(c_{2} \gamma\right)_{\psi}^{\prime}\right]+c_{2} \mid \nabla \psi\left((\beta)_{\theta}^{\prime}-c_{2} \mid \nabla \psi\left(c_{3} \gamma\right)_{\psi}^{\prime}-\frac{\xi_{12}}{J}\left(c_{2} \gamma\right)_{\theta}^{\prime}-\frac{\xi_{12}}{J}\left(c_{3} \gamma\right)_{\varphi}^{\prime}\right. \\
\gamma_{23}=-\frac{\xi_{22}}{J}\left(c_{3} g_{33}+c_{2} g_{23}\right)_{\psi}^{\prime}+\frac{\xi_{22}}{J}\left(c_{2} g_{12}+c_{3} g_{13}\right)_{\varphi}^{\prime}-c_{2} \mid \nabla \psi\left(c_{2} g_{22}+c_{3} g_{23}\right)_{\psi}^{\prime}+ \\
c_{2}|\nabla \psi|\left(c_{2} g_{12}+c_{3} g_{13}\right)_{\theta}^{\prime}+\frac{\xi_{12}}{J}\left(c_{3} g_{33}+c_{2} g_{23}\right)_{\theta}^{\prime}-\frac{\xi_{12}}{J}\left(c_{2} g_{22}+c_{3} g_{23}\right)_{\varphi}^{\prime}
\end{array}
\end{aligned}
$$

Curl component along the total magnetic field is:

$$
\begin{gathered}
\left.\operatorname{rot} \vec{a}\right|_{b}=\varsigma_{31} \frac{\partial a_{2}}{\partial \psi}+\varsigma_{32} \frac{\partial a_{3}}{\partial \psi}+\varsigma_{33} \frac{\partial a_{3}}{\partial \theta}+\varsigma_{34} \frac{\partial a_{2}}{\partial \theta}+\varsigma_{35} \frac{\partial a_{1}}{\partial \theta}+\varsigma_{36} \frac{\partial a_{3}}{\partial \varphi}+\varsigma_{37} \frac{\partial a_{21}}{\partial \varphi}+\varsigma_{38} \frac{\partial a_{1}}{\partial \varphi}+ \\
\gamma_{31} a_{1}+\gamma_{32} a_{2}+\gamma_{33} a_{3}
\end{gathered}
$$


where

$$
\begin{aligned}
& \varsigma_{31}=\frac{\gamma}{J}\left(c_{3} \xi_{33}+c_{2} \xi_{23}\right)=\frac{\gamma}{J} \\
& \varsigma_{32}=0 \\
& \varsigma_{33}=0 \\
& \varsigma_{34}=-\frac{1}{J \gamma}\left(g_{12} g_{33}-g_{13} g_{23}\right) \\
& \varsigma_{35}=-\frac{1}{\gamma}\left(c_{3} g_{33}+c_{2} g_{23}\right) \\
& \varsigma_{36}=0 \\
& \varsigma_{37}=\frac{1}{\gamma J}\left(g_{12} g_{23}-g_{22} g_{13}\right) \\
& \varsigma_{38}=\frac{1}{\gamma}\left(c_{2} g_{22}+c_{3} g_{23}\right) \\
& \gamma_{31}=\frac{1}{J}\left\{\xi_{23}(v)_{\varphi}^{\prime}-\xi_{33}(v)_{\theta}^{\prime}\right\} \\
& \gamma_{32}=\frac{1}{J}\left\{-\xi_{13}\left[\left(c_{2} \gamma\right)_{\theta}^{\prime}+\left(c_{3} \gamma\right)_{\varphi}^{\prime}\right]+\xi_{23}\left[\left(c_{2} \gamma\right)_{\psi}^{\prime}+(\beta)_{\varphi}^{\prime}\right]+\xi_{33}\left[\left(c_{3} \gamma\right)_{\psi}^{\prime}-(\beta)_{\theta}^{\prime}\right]\right\} \\
& \gamma_{33}=\frac{1}{J}\left\{\begin{array}{l}
-\xi_{13}\left[\left(c_{2} g_{22}+c_{3} g_{23}\right)_{\varphi}^{\prime}-\left(c_{3} g_{33}+c_{2} g_{23}\right)_{\theta}^{\prime}\right]+\xi_{23}\left[\left(c_{2} g_{12}+c_{3} g_{13}\right)_{\varphi}^{\prime}-\left(c_{3} g_{33}+c_{2} g_{23}\right)_{\psi}^{\prime}\right]+ \\
\xi_{33}\left[\left(c_{2} g_{22}+c_{3} g_{23}\right)_{\psi}^{\prime}-\left(c_{2} g_{12}+c_{3} g_{13}\right)_{\theta}^{\prime}\right]
\end{array}\right\}
\end{aligned}
$$

The above curl components are used in construction of ROT_ROT E expressions (containing about 100 terms for each $\psi, \theta, \varphi$ components, and are not listed here due to this reason).

\subsection{Divergence operator}

The divergence of any vector $\mathbf{A}$ has a simple form for its contravariant components:

$$
\operatorname{Jiv} \vec{A}=\frac{\partial\left(J A^{s}\right)}{\partial s}+\frac{\partial\left(J A^{\theta}\right)}{\partial \theta}+\frac{\partial\left(J A^{\varphi}\right)}{\partial \varphi}
$$


Transforming contravariant components to the local electrical field components $E_{s}, E_{N}$, $\mathrm{E}_{\mathrm{b}}$ (using above formulas (1.2)) one obtains for stellarators (and for particular 2D tokamak case) :

$$
\begin{aligned}
\operatorname{div} \vec{E}= & \frac{1}{J} \frac{\partial\left(J|\nabla \psi| E_{s}\right)}{\partial s}+ \\
& \frac{1}{J} \frac{\partial}{\partial \theta}\left[-\frac{1}{|\nabla \psi| J}\left(g_{12} g_{33}-g_{13} g_{23}\right) E_{s}+\frac{1}{|\nabla \psi|}\left(c_{3} g_{33}+c_{2} g_{23}\right) E_{N}+J c_{2} E_{b}\right]+ \\
& \frac{1}{J} \frac{\partial}{\partial \varphi}\left[\frac{1}{|\nabla \psi| J}\left(g_{12} g_{23}-g_{22} g_{13}\right) E_{s}-\frac{1}{|\nabla \psi|}\left(c_{2} g_{22}+c_{3} g_{23}\right) E_{N}+J c_{3} E_{b}\right]
\end{aligned}
$$

During descretization of divergence operator in the code a more compact form is used:

$$
\begin{aligned}
\operatorname{div} \vec{E}= & \frac{1}{J} \frac{\partial\left(J \alpha_{11} E_{s}\right)}{\partial s}+ \\
& \frac{1}{J} \frac{\partial}{\partial \theta}\left[J \alpha_{21} E_{s}+J \alpha_{22} E_{N}+J \alpha_{23} E_{b}\right]+ \\
& \frac{1}{J} \frac{\partial}{\partial \varphi}\left[J \alpha_{31} E_{s}+J \alpha_{32} E_{N}+J \alpha_{33} E_{b}\right]
\end{aligned}
$$

where

$$
\begin{aligned}
& \alpha_{11}=|\nabla \psi| \\
& \alpha_{21}=-\frac{1}{|\nabla \psi| J^{2}}\left(g_{12} g_{33}-g_{13} g_{23}\right) \\
& \alpha_{22}=+\frac{1}{|\nabla \psi| J}\left(c_{3} g_{33}+c_{2} g_{23}\right) \\
& \alpha_{23}=c_{2} \\
& \alpha_{31}=\frac{1}{|\nabla \psi| J^{2}}\left(g_{12} g_{23}-g_{22} g_{13}\right) \\
& \alpha_{32}=-\frac{1}{|\nabla \psi| J}\left(c_{2} g_{22}+c_{2} g_{23}\right) \\
& \alpha_{33}=c_{3}
\end{aligned}
$$

\subsection{Gradient operator}

Starting from simple gradient expression in contravariant basis vectors it is easy to 
obtain the gradient operator over our chosen local orthogonal basis $\hat{e}_{s}, \hat{e}_{N}, \hat{e}_{b}$ :

$$
\begin{aligned}
\nabla \Phi= & \hat{e}_{s}\left\{|\nabla \psi| \frac{\partial \Phi}{\partial \psi}-\frac{1}{\gamma J}\left(g_{12} g_{33}-g_{13} g_{23}\right) \frac{\partial \Phi}{\partial \theta}+\frac{1}{\gamma J}\left(g_{12} g_{23}-g_{22} g_{13}\right) \frac{\partial \Phi}{\partial \varphi}\right\}+ \\
& \hat{e}_{N}\left\{\frac{1}{J|\nabla \psi|}\left(c_{3} g_{33}+c_{2} g_{23}\right) \frac{\partial \Phi}{\partial \theta}-\frac{1}{J|\nabla \psi|}\left(c_{2} g_{22}+c_{3} g_{23}\right) \frac{\partial \Phi}{\partial \varphi}\right\}+ \\
& \hat{e}_{b}\left\{c_{2} \frac{\partial \Phi}{\partial \theta}+c_{3} \frac{\partial \Phi}{\partial \varphi}\right\}
\end{aligned}
$$

Here, as above,

$$
\gamma=\left(g_{22} g_{33}-g_{23}^{2}\right)^{1 / 2}
$$

and part of coefficients is expressed through the above $\alpha_{\mathrm{ij}}$.

\subsection{Fourier decomposition}

To solve eqs.(1.1) the electrical fields ( $\mathrm{E} \equiv \mathrm{A}$ ) are expanded in Fourier series to describe their $\theta$ and $\varphi$ dependencies. Since the equilibrium is not axis symmetric, the toroidal modes couple and can not be treated independently. Assuming that the equilibrium and the antenna generally are not up-down symmetrical about plane $Z=0$, we expand the fields in series of the form:

$$
A(\psi, \theta, \varphi)=\sum_{m n} a_{m n}(\psi) e^{i(m \theta+n \varphi)}
$$

The equilibrium quantities are varied in toroidal direction with the periodicity of the toroidal and/or helical coils, described for a concrete machine by the number $\mathrm{N}_{\mathrm{P}}$ of a magnetic field periods. Respectively, we expand the equilibrium quantities in the double periodic Fourier series of the form:

$$
g(\psi, \theta, \varphi)=\sum_{m n} g_{m n}(\psi) e^{i\left(m \theta+n^{\prime} N_{p} \varphi\right)}
$$

Fourier analysis of eqs. (3.1)-(3.2) then leads to the system of differential equations in quasi radial coordinate, $\psi$. These equations we will write below in explicit form. The appearing convolution sums will be calculated by fast Fourier transform method. 
We stress that due to double toroidal periodicity in stellarators the toroidal harmonics are coupled through the condition:

$$
n^{\prime} N_{p}+n-k=0
$$

being some kind analog of axis symmetry of tokamaks reflected to the stellarators.

\subsection{Spatial differencing}

After Fourier decomposition in $\theta$ and $\varphi$ we set up a finite difference grid in $\psi$ only. Critical moment for that descretization scheme is that for vector A, the identity $\operatorname{div}(\operatorname{rot} \mathrm{A})$ $=0$ must be hold identically by numerical difference operators. We state that RF induced current, rotrotA in eqs.(1.1-1.1'), is divergence free vector. Here we satisfy to that requirement introducing the mesh scheme, where we set up the system of non equally, in general, spaced "integer" surfaces with $\psi_{\mathrm{j}}$, and a set of "half integer" surfaces, where $\psi_{\mathrm{j}-}$ ${ }_{1 / 2}=\left(\psi_{j}+\psi_{j-1}\right) / 2$. The integer surface $\mathrm{j}=0$ is defined as magnetic axis, while that with $\mathrm{j}$ $=\mathrm{N}$ is the conducting chamber, surrounding the plasma. We state, that components $\mathrm{a}_{\theta}$ and $a_{\varphi}$ are defined on "integer" surfaces, while $a_{\psi}$ is defined at the half points. Then components $\mathrm{J}^{\theta}, \mathrm{J}^{\varphi}$ of induced plasma currents (respectively, rotrotA) of derived vectors are centred at the "integer" surfaces, while the $\mathrm{J}^{\psi}$ (and $\left.\operatorname{rotrot}\right|^{\psi}{ }^{\psi}$ ) are centred at intermediate surfaces.

Now we install the expression eq.(3.2.1) into eqs.(1.1)-(1.1'), assign plasma response and antenna currents in eq.(1.1) as being for brevity RHS, multiply both sides of equations by $\exp \{-\mathrm{i}(l \theta+\mathrm{k} \varphi)$ ( $l$ and $\mathrm{k}$ are integer numbers) and integrate over angles $\varphi$ and $\theta$. Then one obtains coupled system of differential equations. With $a_{s}$ defined at the half points and $\mathrm{a}_{\theta}, \mathrm{a}_{\varphi}$ at the integer points, we make use second order accurate centred difference formulae to derive complicated but straightford system of linear algebraic equations.

\section{PLASMA RESPONSE}

\subsection{Zero Larmor radius current $J^{(0)}$}

Plasma wave induced current $\boldsymbol{J}^{(0)}$ response, when finite Larmor radius effects are ignored, is calculated by usual way through the hot dielectric tensor as will be shown below when discussing ORA code option keeping $\left(k_{\perp} \rho_{e, i}\right)^{2}$ terms equal to zero. For 
present goals however also is the useful some another representation, similar [5], through integral operators $\hat{L}, \hat{R}, \hat{P}$ :

$$
\begin{aligned}
\vec{J}^{(0)} & =\frac{\omega}{4 \pi i}\left(-\vec{E}+\hat{L} E_{+} \vec{u}_{+}+\hat{R} E_{\beth} \overrightarrow{u_{-}}+\hat{P} E_{b} \hat{e}_{b}\right) \\
& =\frac{\omega}{4 \pi i}\left(\left(\hat{S} E_{\psi}-i \hat{D} E_{N}\right) \hat{e}_{b}+\left(i \hat{D} E_{\psi}+\hat{S} E_{N}\right) \hat{e}_{N}+\hat{P} E_{b} \hat{e}_{b}\right)
\end{aligned}
$$

where

$$
\begin{aligned}
& \hat{L} E_{+}=E_{+}(\vec{r})-\sum_{\alpha} \frac{\omega_{p \alpha}^{2}}{\omega_{c \alpha}^{2}} \frac{v_{T \alpha}^{2}}{c^{2}} \int_{-\infty}^{+\infty} d u \frac{e^{-u^{2}}}{\sqrt{\pi}}\left(-i \omega \int_{-\infty}^{t} d t^{\prime} e^{i \int_{t^{\prime}}^{t}\left(\omega-\omega_{g}^{\prime}\right) d \tau} E_{+}{ }^{\prime}\right) \\
& \hat{R} E_{-}=E_{-}(\vec{r})-\sum_{\alpha} \frac{\omega_{p \alpha}^{2}}{\omega_{c \alpha}^{2}} \frac{v_{T \alpha}^{2}}{c^{2}} \int_{-\infty}^{+\infty} d u \frac{e^{-u^{2}}}{\sqrt{\pi}}\left(-i \omega \int_{-\infty}^{t} d t^{\prime} e^{i \int_{t^{\prime}}^{\prime}\left(\omega-\omega_{g}^{\prime}\right) d \tau} E_{-}{ }^{\prime}\right) \\
& \hat{P} E_{b}=E_{b}(\vec{r})-\sum_{\alpha} \frac{\omega_{p \alpha}^{2}}{\omega_{c \alpha}^{2}} \frac{v_{T \alpha}^{2}}{c^{2}} \int_{-\infty}^{+\infty} d u \frac{e^{-u^{2}}}{\sqrt{\pi}}\left(-i \omega \int_{-\infty}^{t} d t^{\prime} e^{i \omega\left(t-t^{\prime}\right)} E_{b}{ }^{\prime}\right)
\end{aligned}
$$

and summation is over plasma particles (electrons and ions) $\alpha=\mathrm{e}, \mathrm{i}$ and

$$
\hat{S}=\frac{1}{2}(\hat{R}+\hat{L}), \quad \hat{D}=\frac{1}{2}(\hat{R}-\hat{L})
$$

The t'-integrations are along magnetic field lines for each species

$$
\vec{E}^{\prime}=\vec{E}\left(\vec{r}-\int_{t^{\prime}}^{t}\left(v_{/} \hat{e}_{b}\right) d \tau\right)
$$

\subsection{Ion FLR current}

In the code the approximation derived by Swanson [9] and by Colestock and Kashuba (SCK) [10], augmented by the appropriate FLR terms for the electrons [5], is used. The only terms resonant at $\omega=2 \omega_{c i}$ are retained in the FLR ion current. In vector form ion finite Larmor current is given [5] by

$$
\begin{aligned}
\vec{J}^{(2)}=\frac{c^{2}}{4 \pi i \omega} \vec{R}\left\{\nabla_{\perp}\left(\sigma^{(2)} \nabla_{\perp}\left(\vec{R} \vec{E}_{\perp}\right)-i \delta^{(2)} \nabla_{\perp}\left(\hat{e}_{b} \times \vec{R} \vec{E}_{\perp}\right)\right)-\right. \\
\left.\left(\hat{e}_{b} \times \nabla_{\perp}\right)\left(\sigma^{(2)} \nabla_{\perp}\left(\hat{e}_{b} \times \vec{R} \vec{E}_{\perp}\right)+i \delta^{(2)} \nabla_{\perp}\left(\vec{R} \vec{E}_{\perp}\right)\right)\right\}
\end{aligned}
$$

where 


$$
\sigma^{(2)}=\frac{\rho^{(2)}+\lambda^{(2)}}{2}, \quad \delta^{(2)}=\frac{\rho^{(2)}-\lambda^{(2)}}{2}
$$

with

$$
\begin{aligned}
& \lambda^{(2)} E_{+}=\frac{1}{2} \sum_{i} \frac{\omega_{p i}^{2}}{\omega_{c i}^{2}} \frac{v_{T i}^{2}}{c^{2}} \int_{-\infty}^{+\infty} d u \frac{e^{-u^{2}}}{\sqrt{\pi}}\left(-i \omega \int_{-\infty}^{t} d t^{\prime} e^{i \int_{t^{\prime}}^{t}\left(\omega-2 \omega_{g}^{\prime}\right) d \tau} E_{+}{ }^{\prime}\right) \\
& \rho^{(2)} E_{-}=\frac{1}{2} \sum_{i} \frac{\omega_{p i}^{2}}{\omega_{c i}^{2}} \frac{v_{T i}^{2}}{c^{2}} \int_{-\infty}^{+\infty} d u \frac{e^{-u^{2}}}{\sqrt{\pi}}\left(-i \omega \int_{-\infty}^{t} d t^{\prime} e^{i \int_{t^{\prime}}^{t}\left(\omega+2 \omega_{g}^{\prime}\right) d \tau} E_{-}{ }^{\prime}\right)
\end{aligned}
$$

The matrix $\vec{R}=\vec{R}^{-1}$ is the reflection matrix with respect to the plane containing $B_{0}$ : with $\vec{u}_{g}=\nabla_{\perp} B_{0} / \nabla_{\perp} B_{0} \mid$ it can be written

$$
\vec{R} \vec{E}_{\perp}=\vec{E}_{\perp}-2 \vec{u}_{g} \times\left(\vec{E}_{\perp} \times \vec{u}_{g}\right)
$$

where for $3 \mathrm{D}$ case

$$
\vec{u}_{g}=\hat{e}_{1}\left\{\alpha_{11} \frac{\partial B_{0}}{\partial \psi}+\alpha_{21} \frac{\partial B_{0}}{\partial \theta}\right\} / \nabla_{\perp} B_{0}\left|+\hat{e}_{N}\left\{\alpha_{22} \frac{\partial B_{0}}{\partial \theta}+\alpha_{32} \frac{\partial B_{0}}{\partial \varphi}\right\} /\right| \nabla_{\perp} B_{0} \mid
$$

or in more compact form as

$$
\vec{u}_{g}=a \hat{e}_{1}+b \hat{e}_{N}
$$

The $\vec{R} \vec{E}_{\perp}$ components can be expressed as

$$
\begin{aligned}
\left(\vec{R} \vec{E}_{\perp}\right)_{\psi} & =C_{2} E_{s}+S_{2} E_{N} \\
\left(\vec{R} \vec{E}_{\perp}\right)_{N} & =S_{2} E_{s}-C_{2} E_{N}
\end{aligned}
$$

with coefficients

$$
C_{2}=a^{2}-b^{2}, \quad S_{2}=2 a b
$$

Here according to (4.2.4)

$$
a=\left(\alpha_{11} \frac{\partial B_{0}}{\partial \psi}+\alpha_{21} \frac{\partial B_{0}}{\partial \theta}\right) /\left|\nabla_{\perp} B_{0}\right| \quad, \quad b=\left(\alpha_{22} \frac{\partial B_{0}}{\partial \theta}+\alpha_{32} \frac{\partial B_{0}}{\partial \varphi}\right) /\left|\nabla_{\perp} B_{0}\right|
$$

with $\alpha_{\mathrm{ij}}$ given by (2.2.1). Derivatives of $\mathrm{C}_{2}$ and $\mathrm{S}_{2}$ involve the second derivatives of $\mathrm{B}_{0}$. 


\subsection{Electron FLR current}

The SCK model is completed by taking into account the FLR current, which accounts to two terms

$$
\vec{J}_{e}^{(2)}=\vec{J}_{T T M P}^{(2)}+\vec{J}_{C R O S S}^{(2)}
$$

describing the magnetic pumping (TTMP) and mixing with electron Landau damping (ELD)

$$
\begin{aligned}
& \vec{J}_{\text {TTMP }}^{(2)}=-\frac{c^{2}}{2 \pi i \omega} \nabla_{\perp} \times\left(\lambda^{(0)} \nabla_{\perp} \times\left(\nabla_{\perp} \times \vec{E}_{\perp}\right)\right) \\
& \vec{J}_{\text {CROSS }}^{(2)}=\frac{c^{2}}{4 \pi \omega}\left\{\nabla_{\perp} \times\left(\xi^{(0)}\left(\hat{e}_{b} \nabla\right) E_{b} \hat{e}_{b}\right)+\hat{e}_{b}\left(\hat{e}_{b} \nabla\right)\left(\xi^{(0)}\left(\hat{e}_{b} \nabla_{\perp} \times \vec{E}_{\perp}\right)\right)\right\}
\end{aligned}
$$

where

$$
\begin{aligned}
\lambda^{(0)} \vec{E} & =\frac{1}{2} \frac{\omega_{p e}^{2}}{\omega_{c e}^{2}} \frac{v_{T e}^{2}}{c^{2}} \int_{-\infty}^{+\infty} d u \frac{e^{-u^{2}}}{\sqrt{\pi}}\left(-i \omega \int_{-\infty}^{t} d t^{\prime} e^{i \omega\left(t-t^{\prime}\right)} \vec{E}^{\prime}\right) \\
\xi^{(0)} \vec{E} & =\frac{1}{2} \frac{\omega_{p e}^{2}}{\omega \omega_{c e}} \frac{v_{T e}^{2}}{c^{2}} \int_{-\infty}^{+\infty} d u \frac{e^{-u^{2}}}{\sqrt{\pi}}\left(\omega^{2} \frac{\partial}{\partial \omega} \int_{-\infty}^{t} d t^{\prime} e^{i \omega\left(t-t^{\prime}\right)} \vec{E}^{\prime}\right)
\end{aligned}
$$

We remark the omission of the FLR ion terms resonant at the fundamental ion cyclotron frequency. These terms are always small corrections to the zero Larmor radius current, which is also resonant at $\omega=\omega_{c i}$, in contrast to the FLR terms which have been kept, which are resonant near second harmonic where $\boldsymbol{J}^{(\boldsymbol{0})}$ remains finite one. Extended argumentation can be found in $[5,6]$.

\subsection{Order reduction algorithm (ORA)}

The RF induced currents in ORA algorithm (this option is included in the code) are described by the dielectric tensor $\vec{\varepsilon}$, the simplest famous form of which is naturally realized in above local special coordinate system $\left\{\hat{e}_{s}, \hat{e}_{N .}, \hat{e}_{b}\right\}$.

In this system the right hand side of basic equation (1.1), related to the plasma response, is written as 


$$
\begin{aligned}
R H S= & \frac{\nabla s}{|\nabla s|}\left(\bar{\varepsilon}_{11} E_{s}+\bar{\varepsilon}_{12} E_{N}+\bar{\varepsilon}_{13} E_{b}\right)+\widehat{e}_{b}\left(\bar{\varepsilon}_{21} E_{s}+\bar{\varepsilon}_{22} E_{N}+\bar{\varepsilon}_{23} E_{b}\right)+\widehat{e}_{h}\left(\bar{\varepsilon}_{31} E_{s}+\bar{\varepsilon}_{32} E_{N}+\bar{\varepsilon}_{33} E_{b}\right) \equiv \\
& D_{1} \widehat{e}_{s}+D_{2} \widehat{e}_{N}+D_{3} \widehat{e}_{b}
\end{aligned}
$$

where we for simplicity introduced dimensional tensor $\bar{\varepsilon}=k_{0}^{2} \vec{\varepsilon}$.

More compactly wave the induced plasma current is expressed through plasma conductivity tensor as

$$
\vec{j}_{p}(s, \theta, \varphi)=\sum_{m n} \vec{\sigma}\left(s, \theta, \varphi, k_{\mathbf{I}}^{m n}\right) \vec{E}_{m n}(s) e^{i(m \theta+n \varphi)}
$$

where $\vec{E}_{m n}$ are poloidal and toroidal harmonics of the wave electric field. The dielectric tensor, connected with conductivity one by simple relation, is expanded to second order in Larmor radius

$$
\vec{\varepsilon}=\vec{\varepsilon}^{(0)}+i k_{\perp} \vec{\varepsilon}^{(1)}-k_{\perp}^{2} \vec{\varepsilon}^{(2)}
$$

where in local Stix's coordinates the tensor elements [8] are

$$
\begin{aligned}
& \varepsilon_{11}^{(0)}=1+\frac{1}{2} \sum_{\alpha}\left(\widetilde{Z}_{1}^{(\alpha)}+\widetilde{Z}_{-1}^{(\alpha)}\right) \\
& \varepsilon_{22}^{(0)}=\varepsilon_{11}^{(0)} \\
& \varepsilon_{12}^{(0)}=\frac{i}{2} \sum_{\alpha}\left(\widetilde{Z}_{1}^{(\alpha)}-\widetilde{Z}_{-1}^{(\alpha)}\right)=-\varepsilon_{21}^{(0)} \\
& \varepsilon_{33}^{(0)}=1+\frac{2}{\left(k_{/ /} V_{T \alpha}\right)^{2}} \sum_{\alpha}\left(\omega_{p \alpha}^{2}+\omega^{2} \widetilde{Z}_{0}^{(\alpha)}\right) \\
& \varepsilon_{13}^{(1)}=-i \sum_{\alpha} \frac{1}{2 k_{/ /} \omega_{c \alpha}}\left[\left(\omega-\omega_{c \alpha}\right) \widetilde{Z}_{1}^{(\alpha)}-\left(\omega+\omega_{c \alpha}\right) \widetilde{Z}_{-1}^{(\alpha)}\right]=\varepsilon_{31}^{(1)} \\
& \varepsilon_{23}^{(1)}=\sum_{\alpha} \frac{1}{2 k_{/ /} \omega_{c \alpha}}\left[2 \omega \widetilde{Z}_{0}^{(\alpha)}-\left(\omega-\omega_{c \alpha}\right) \widetilde{Z}_{1}^{(\alpha)}-\left(\omega+\omega_{c \alpha}\right) \widetilde{Z}_{-1}^{(\alpha)}\right]=-\varepsilon_{32}^{(1)} \\
& \varepsilon_{11}^{(2)}=-\sum_{\alpha} \frac{V_{T \alpha}^{2}}{4 \omega_{c \alpha}^{2}}\left(\widetilde{Z}_{2}^{(\alpha)}+\widetilde{Z}_{-2}^{(\alpha)}-\widetilde{Z}_{1}^{(\alpha)}-\widetilde{Z}_{-1}^{(\alpha)}\right) \\
& \varepsilon_{12}^{(2)}=-i \sum_{\alpha} \frac{V_{T \alpha}^{2}}{4 \omega_{c \alpha}^{2}}\left(\widetilde{Z}_{2}^{(\alpha)}-\widetilde{Z}_{-2}^{(\alpha)}-2 \widetilde{Z}_{1}^{(\alpha)}+2 \widetilde{Z}_{-1}^{(\alpha)}\right)=-\varepsilon_{21}^{(2)}
\end{aligned}
$$




$$
\begin{aligned}
& \varepsilon_{22}^{(2)}=-\sum_{\alpha} \frac{V_{T \alpha}^{2}}{4 \omega_{c \alpha}^{2}}\left(4 \widetilde{Z}_{0}^{(\alpha)}-3 \widetilde{Z}_{1}^{(\alpha)}-3 \widetilde{Z}_{-1}^{(\alpha)}+\widetilde{Z}_{2}^{(\alpha)}+\widetilde{Z}_{-2}^{(\alpha)}\right) \\
& \varepsilon_{33}^{(2)}=-\sum_{\alpha} \frac{1}{2\left(k_{/ /} \omega_{c \alpha}\right)^{2}}\left[-2 \omega^{2} \widetilde{Z}_{0}^{(\alpha)}+\left(\omega-\omega_{c \alpha}\right)^{2} \widetilde{Z}_{1}^{(\alpha)}+\left(\omega+\omega_{c \alpha}\right)^{2} \widetilde{Z}_{-1}^{(\alpha)}\right]
\end{aligned}
$$

Here $\widetilde{Z}_{n}^{(\alpha)}$ is the scaled plasma dispersion function:

$$
\widetilde{Z}_{n}^{(\alpha)}=\frac{\omega_{p \alpha}^{2}}{\omega k_{/ /} V_{T \alpha}} Z\left(\frac{\omega-n \omega_{c \alpha}}{\left|k_{/ /}\right| V_{T \alpha}}\right),
$$

with $V_{T \alpha}=\sqrt{2 k_{B} T_{\alpha} / M_{\alpha}}, \omega_{p \alpha}$ and $\omega_{c \alpha}$ being plasma and cyclotron frequencies of species $\alpha$. Summation over $\alpha$ in above formulas comes over electrons and ions in plasma.

Due to finite Larmor radius expansions, only harmonic resonances up to second order are included in Eqs. (4.2 ). Higher order resonances are included according Akhiezer et all [8].

\section{PARALLEL WAVE NUMBER}

The plasma wave induced currents or dielectric tensor $\vec{\varepsilon}\left(s, \theta, \varphi, k_{\|}^{m n}\right)$, appearing e.g. in eqs.(4.2), depends on $k_{\mathbf{I}}^{m n}$ through toroidal plasma dispersion function $Z((\omega$ $\left.\mathrm{N} \Omega_{\mathrm{ce}, \mathrm{i}}\right) / \mathrm{k}_{/ /}^{\mathrm{mn}} \mathrm{v}_{\mathrm{Te}, \mathrm{i}}$ ), where $\mathrm{N}$ is the cyclotron harmonic number. To account the effect of the poloidal magnetic field influence on $\mathrm{k}_{/ /}$wave number formation it is usually considered that the $\mathrm{k}_{/ /}$is the component of the spatial gradient in the direction of $\mathrm{B}_{0}$. The gradient operator in a non orthogonal system is

$$
\nabla=\nabla s \frac{\partial}{\partial s}+\nabla \theta \frac{\partial}{\partial \theta}+\nabla \varphi \frac{\partial}{\partial \varphi}
$$

And we define $\mathrm{k}_{/ /}$as:

$$
i k_{/ /}=\widehat{e}_{b} \bullet \nabla=\widehat{e}_{b}\left(\nabla \theta \frac{\partial}{\partial \theta}+\nabla \varphi \frac{\partial}{\partial \varphi}\right)
$$

Expanding $\hat{e}_{b}$ over unit vectors $\mathrm{e}_{\varphi}$ and $\mathrm{e}_{\theta}$

$$
\hat{e}_{b}=\hat{e}_{\varphi} \frac{B^{\varphi}}{|B|}+\widehat{e}_{\theta} \frac{B^{\theta}}{|B|}
$$


Finally we have

$$
k_{\mathbf{1}}=n \frac{B^{\varphi}}{|B|}+m \frac{B^{\theta}}{|B|}
$$

where

$$
|B|^{2} \equiv B^{i} B_{i}=\left(B^{\theta}\right)^{2} g_{22}+2 B^{\theta} B^{\varphi} g_{23}+\left(B^{\varphi}\right)^{2} g_{33}
$$

This equation (5.2) generalizes the $\mathrm{k}_{/ /}$definition for a stellarator magnetic field geometry. The tokamak $\mathrm{k}_{/ /}$case is easily obtained from eq.(5.2). In this equation (5.2) the poloidal field dominates the $\mathrm{k}_{/ /}$variation when

$$
|m| \geq n \frac{B^{\varphi}}{B^{\theta}}
$$

\subsection{Orbit integrals}

Modes expansion method permits to analytically treat integrals along the parallel trajectories of the particles in equations (4.1.2), (4.2.3) and (4.3.2). For example, in the zero Larmor radius terms in the wave induced plasma current one has

$$
\hat{L} E_{+}=\sum_{m, l} \hat{L}\left(\psi, \theta, \varphi, k_{/ l}^{m l}\right) E_{+}^{m l}
$$

with

$$
\hat{L}\left(\psi, \theta, \varphi, k_{/ /}^{m l}\right)=1-\sum_{\alpha} \frac{\omega_{p \varepsilon}^{2}}{\omega^{2}}\left(-x_{0 \alpha} \hat{Z}\left(x_{1 \alpha}\right)\right)
$$

Here $\hat{Z}$ is so called toroidal plasma dispersion function (TPDF)

$$
-x_{0 \alpha} \hat{Z}\left(x_{n \alpha}\right)=\int_{-\infty}^{+\infty} d u \frac{e^{-u^{2}}}{\sqrt{\pi}}\left(-i \omega \int_{-\infty}^{t} d t^{\prime} e^{i \int_{t^{t}}^{t} k_{\|}^{m l} V_{T}\left(x_{n \alpha}^{\prime \prime}-u^{\prime \prime}\right) d t^{\prime \prime}}\right)
$$

with argument

$$
x_{n \alpha}=x_{n \alpha}(m, l, \psi, \theta, \varphi)=\frac{\omega-n \omega_{c \alpha}}{k_{/ /}^{m l} V_{T \alpha}}
$$

where for stellarator geometry is used (5.2): 


$$
k_{/ /}^{m l}(\psi, \theta, \varphi)=k_{/ /}^{m l}=l \frac{B^{\varphi}}{|B|}+m \frac{B^{\theta}}{|B|}
$$

The operators $\hat{R}$ and $\hat{P}$ are similarly expressed in terms of TPDF of arguments $x_{-1 \alpha}, x_{0 \alpha}$, respectively and the FLR operators $\hat{\lambda}^{(2)}$ and $\hat{\rho}^{(2)}$ are similarly expressed in terms of TPDF of arguments $x_{ \pm 2, \alpha}$.

For large arguments, $\left|x_{n}\right|>>1, \hat{Z}$ has asymptotic behaviour as plasma dispersion function of the uniform plasma

$$
Z\left(x_{n}\right)=\frac{1}{\sqrt{\pi}} \int_{-\infty}^{+\infty} \frac{e^{-u^{2}}}{u-x_{n}} d u+i \sqrt{\pi} e^{-x_{n}^{2}}
$$

to which it reduces exactly in the limit of a uniform magnetic field.

Broad discussion of TPDF can be found in [11]. There is also proposed to use the "effective" parallel wavenumber while working with classical PDF $Z\left(x_{n}\right)$.

\section{BOUNDARY CONDITIONS}

- Plasma is surrounded by perfectly conducting wall

Boundary condition on conducting wall

Tangential components of $\mathbf{E}$ vanishes

Boundary condition on magnetic axis $(\psi=0)$ :

$$
\left.\vec{E}\left(s=s_{\text {wall }}, \theta, \varphi\right)\right)_{\tan g}=0
$$

The only the $\theta$ - and $\varphi$ - components of $\mathrm{E}$ are required at the axis $\psi=0$; one has

$$
\begin{aligned}
& E_{\theta}^{m n}=0, \text { all } \mathrm{m} \text { and } \mathrm{n}, \\
& E_{\varphi}^{m n}=0 \quad \text { if } \mathrm{m} \neq 0,
\end{aligned}
$$




$$
\frac{\partial E_{\varphi}^{m n}}{\partial \psi}=0 \quad \text { if } \mathrm{m}=0
$$

\section{ABSORBED POWER}

\subsection{Fundamental harmonic ion cyclotron absorption}

The absorbed power at first (fundamental) ion cyclotron harmonic, averaged over magnetic surface, is given by

$$
P_{i}^{1}(\psi)=\frac{\omega}{4 \pi} \operatorname{Re} \sum_{m, l} \sum_{m^{\prime}, l^{\prime}} \int J e^{i\left[\left(m^{\prime}-m\right) \theta+\left(l^{\prime}-l\right) \varphi\right]}\left\{E_{+}^{(m, l)^{*}}(\psi) \operatorname{Im}\left(L\left(\psi, \theta, \varphi, k_{/ l}^{m^{\prime}, l^{\prime}}\right)\right) E_{+}^{\left(m^{\prime}, l^{\prime}\right)}(\psi)\right\} d \theta d \varphi
$$

\subsection{Second harmonic ion cyclotron absorption}

The absorbed power at second ion cyclotron harmonic, averaged over magnetic surface, is given by

$$
\begin{aligned}
P_{i}^{2}(\psi)=- & \frac{\omega}{8 \pi} \operatorname{Re} \sum_{m, l} \sum_{m^{\prime}, l^{\prime}} \frac{c^{2}}{\omega^{2}} \int J e^{i\left[\left(m^{\prime}-m\right) \theta+\left(l^{\prime}-l\right) \varphi\right]}\left\{\nabla_{\perp}\left(\left(\vec{R} \vec{E}_{\perp}^{m, l}\right)+i\left(\hat{e}_{b} \times \vec{R} \vec{E}_{\perp}^{m, l}\right)\right)^{*} \times\right. \\
& \operatorname{Im}\left(\hat{\lambda}^{(2)}\left(\psi, \theta, \varphi, k_{\|}^{m^{\prime}, l^{\prime}}\right) \cdot \nabla_{\perp}\left(\left(\vec{R} \vec{E}_{\perp}^{m^{\prime}, l^{\prime}}\right)+i\left(\hat{e}_{b} \times \vec{R} \vec{E}_{\perp}^{m^{\prime}, l^{\prime}}\right)\right)\right\} d \theta d \varphi
\end{aligned}
$$

\subsection{Electron Landau absorption}

The electron Landau absorption averaged over magnetic surface:

$P_{e}^{0}(\psi)=\frac{\omega}{4 \pi} \operatorname{Re} \sum_{m, l} \sum_{m^{\prime}, l^{\prime}} \int J e^{i\left[\left(m^{\prime}-m\right) \theta+\left(l^{\prime}-l\right) \varphi\right]}\left\{E_{b}^{(m, l)^{*}}(\psi) \operatorname{Im}\left(P\left(\psi, \theta, \varphi, k_{/ /}^{m^{\prime}, l^{\prime}}\right)\right) E_{b}^{\left(m^{\prime}, l^{\prime}\right)}(\psi)\right\} d \theta d \varphi$

7.4 Electron transit time absorption

$$
\begin{aligned}
P_{e}^{T T M P}(\psi)=- & \frac{\omega}{4 \pi} \operatorname{Re} \sum_{m, l} \sum_{m^{\prime}, l} \frac{c^{2}}{\omega^{2}} \int J e^{i\left[\left(m^{\prime}-m\right) \theta+\left(l^{-}-l\right) \varphi\right]}\left\{\left(\nabla_{\perp} \times \vec{E}_{\perp}^{m, l}\right)^{*} \times\right. \\
& \operatorname{Im}\left(2 \hat{\lambda}^{(0)}\left(\psi, \theta, \varphi, k_{/ l}^{m^{\prime}, l^{\prime}}\right) \cdot\left(\nabla_{\perp} \times \vec{E}_{\perp}^{m^{\prime}, l^{\prime}}\right)\right\} d \theta d \varphi
\end{aligned}
$$




\subsection{Cross electron absorption}

The cross electron term:

$$
\begin{gathered}
P_{e}^{C R O S S}(\psi)=\frac{\omega}{8 \pi} \operatorname{Re} \sum_{m, l} \sum_{m^{\prime}, l} \frac{c^{2}}{\omega^{2}} \int J e^{i\left[\left(m^{\prime}-m\right) \theta+\left(l^{\prime}-l\right) \varphi\right]}\left\{\left(( \nabla _ { \perp } \times \vec { E } _ { \perp } ^ { m , l } ) ^ { * } \cdot \hat { e } _ { b } \operatorname { I m } \left(\xi_{e}^{0}\left(\psi, \theta, \varphi, k_{/ l}^{m^{\prime}, l^{\prime}}\right)\right.\right.\right. \\
\left.\quad \times\left(k_{/ /}^{m^{\prime} l^{\prime}} E_{b}^{m^{\prime} l^{\prime}}\right)+\left(k_{/ /}^{m l} E_{b}^{m l}\right)^{*} \operatorname{Im}\left(\xi_{e}^{0}\left(\psi, \theta, \varphi, k_{\|}^{m^{\prime} l^{\prime}}\right)\right)\left(\left(\nabla_{\perp} \times \vec{E}_{\perp}^{m^{\prime} l^{\prime}}\right)^{*} \cdot \hat{e}_{b}\right)\right\} d \theta d \varphi
\end{gathered}
$$

\section{Electron damping of ion Bernstein waves}

During ion Bernstein wave propagation away from the mode conversion layer, their wavelength decreases rapidly, until the small Larmor radius approximation finally breaks down. Electron Landau damping of IB waves improves the situation [12]. Similar to $[6,12]$ for stellarator geometry this is done by modifying the imaginary part of the plasma dispersion to account for the IBW damping while leaving the real part unchanged:

$$
\begin{aligned}
& \left.n_{\perp}^{2}\right|_{I B W}=-\frac{n_{/ /}^{2}-S}{\sigma} \\
& \sigma \rightarrow \sigma+i \delta \sigma \\
& \delta \sigma=-i \sigma \frac{\operatorname{Im}\left(\left.n_{\perp}^{2}\right|_{I B W}\right)}{\left.n_{\perp}^{2}\right|_{I B W}}
\end{aligned}
$$

Equation (8.1) describes the mode conversion of Fast Wave to either ion Bernstein wave or Kinetic Alfven wave (also frequently named as ion cyclotron wave (ICW) or slow mode) at ion-ion hybrid layer $n_{/ /}^{2}=S$ in warm plasma. The warm plasma contribution $\sigma$ (formulas 4.2.2, 4.2.3) is modified as shown in Eqn. (8.1), in which a contribution derived from the $k_{\perp}$ root of the full local dispersion relation, retaining all cyclotron harmonics [13], is added to the expression for the perpendicular wave number. In actual implementation in the code, the perpendicular index of refraction $n_{\perp}$ is present only as an

operator. That is, $n_{\perp} \rightarrow \frac{c}{\omega} \nabla_{\perp}$, but warm plasma effects are retained in $\sigma$ as are the damping modifications to it in Eqn. (8.1.3).

\section{Numerical implementation}

The modes expansion method together with the approximations of the toroidal plasma dispersion function discussed above transforms the integrodifferential FLR wave equations into a system of coupled ordinary differential equations in the radial variable $\psi$, whose coefficients are evaluated using the Fast Fourier transform (FFT). The numbers of poloidal $\mathrm{M}$ and toroidal $\mathrm{L}$ Fourier components used in the representation of the coefficients are always a power of two. To reconstruct the fields after solution of the radial equations the inverse Fourier transform is used. 
The construction of the FFT of orders $\mathrm{M}$ and $\mathrm{L}$ requires information from $\mathrm{M}$ equally spaced points in $\theta$ and $\mathrm{L}$ points in $\varphi$. Thus it becomes clear that $\mathrm{M}$ and $\mathrm{L}$ must be sufficient large to get a good resolution of the poloidal and toroidal variations of the resonant $\mathrm{Z}$ functions and simultaneously be sufficient to resolve numerically the shortest wavelength waves occurring in the solution.

The radial $(\psi)$ descretization is accomplished according section 3.3. Finally the descretized system has a three diagonal block structure:

$$
\vec{A} \vec{E}=\vec{J}_{a n t} \text { where } \vec{A}=\left\{\begin{array}{l}
B 2 B 3.0 .0 .0 .0 \\
B 1 B 2 B 3.0 .0 .0 \\
0 . B 1 B 2 B 3.0 .0 \\
0.0 . B 1 B 2 B 3,0 \\
0.0 .0 . B 1 B 2 B 3 \\
0.0 .0 .0 . B 1 B 2
\end{array}\right\}
$$

in which the blocks B1, $\mathbf{B} 2$ and $\mathbf{B 3}$ are each dense matrices of size $O\left(3 N_{M} N_{L}\right)^{2}$. More specifically, the system to be solved has a three diagonal block structure

$$
\mathbf{A}_{\mathrm{i}} \cdot \mathbf{x}_{\mathrm{i}-1}+\mathbf{B}_{\mathrm{i}} \cdot \mathbf{x}_{\mathrm{i}}+\mathbf{C}_{\mathrm{i}} \cdot \mathbf{x}_{\mathrm{i}+1}=\mathbf{y}_{\mathrm{i}} \quad \mathrm{i}=1, \ldots, \mathrm{N}_{\mathrm{p}}
$$

with

$$
\mathbf{A}_{1}=0 \quad \mathbf{C}_{\mathrm{Np}}=0 .
$$

Each $\mathbf{x}_{\mathrm{i}}$ is a $\left(3 N_{M} N_{L}\right)$-dimensional complex vector made up of the Fourier components of E. The code uses Thomas Algorithm (Gaussian inversion specialized for three diagonal system) for parallel processors computer option, distributing matrices across processors, and uses the ScaLapack library and message passing interface (MPI) for parallel matrix operations. The out of core solver is used. The individual blocks are distributed across all processors. Parts of the inverted blocks are stored by local scratch disks. The compact storage methods with out of core solver can also be used for serial code option.

The amount of resolution needed requires more modes to represent the discontinuities such as mode conversion layer and this is also increased by wave dispersion. Shorter wavelength modes will need more radial and poloidal modes. In the case of mode conversion the presence of IBW or KAW implies that perpendicular wave number is on the order of the ion Larmor radius. From the condition $k_{\perp} \rho_{T i} \sim 1$ and approximating $k_{\perp} \sim \frac{m}{r}$ one may estimate the maximum poloidal mode $m \sim r / \rho_{T i}$ number needed in the simulation by specifying the minor radius r. For small machines, like Alcator C-Mod, maximal mode number is significantly greater than poloidal mode numbers for ITER due to larger ion temperatures and smaller magnetic field in that large machine. 


\section{PROGRAM SUMMARY}

Title of program: $\quad$ PSTELION

Operating system: UNIX

Platform: AIX - SP

Programming language used: Fortran 90

Memory required to execute with typical data: Problem dependent;

the test 3D calculation requires about 1.3 Gbyte

No. of bits in a word: 64

Peripherals used: disk

\section{Nature of physical problem}

Code solves the problem of wave excitation, propagation and absorption in 3D stellarator equilibrium high beta plasma in ion cyclotron frequency range and lower. This code is urgently needed for ICRF heating scenarios development for the constructed large W7-X stellarator, NCSX constructed stellarator, and for stellarators being at operation (CHS, W7-AS, LHD etc.) and projected stellarators (ORNL, IPP Garching,....). Code solves the 3D Maxwell- Vlasov antenna-plasma-conducting shell boundary value problem in non-orthogonal flux coordinates $(\psi, \theta, \varphi), \psi$ being magnetic flux function, $\theta$ and $\varphi$ being the poloidal and toroidal angles, respectively. All basic physics, like wave refraction, reflection and diffraction are self consistently included, along with the fundamental ion and ion minority cyclotron resonances, two ion hybrid resonance, electron Landau and TTMP absorption.

This is accomplished in a real confining magnetic field in a plasma major radius direction, in toroidal and poloidal directions, through making use ion and electron Finite Larmor radius effects in wave plasma response second order differential operators. In the Reduced Order Algorithm code option the hot plasma dielectric kinetic tensor is used (FLR effects are accounted through reduced order scheme).

\section{Method of solution}

Code expands the solution in Fourier series over toroidal $(\varphi)$ and poloidal $(\theta)$ angles and solves resulting ordinary differential equations in a radial like $\psi$ - coordinate by finite difference method. The constructed discretization scheme is divergent free one, thus retaining the basic properties of original equations. The Fourier expansion over angles coordinates gives the possibility to correctly construct the "parallel" wave number $\mathrm{K}_{/ /}$and thereby to correctly describe the ICRF waves absorption by a hot plasma.

The toroidal harmonics are tightly coupled with each other due to magnetic field inhomogenity of stellarators in toroidal direction. The code is developed in a manner that includes tokamaks and mirrors as the particular cases through general metric tensor (provided by a plasma equilibrium solver) treatment of the wave equations.

The resulting system of linear equations is solved by making use the ScaLAPACK library of parallelized linear algebra routines and direct use of the MPI interface.

\section{Restrictions on the complexity of the problem}

Code has two versions: STELION1 AND PSTELION. Its tokamak version is also in operation and is actively used for reactor ITER modelling [14] and can be found in 
attached paper [15]. Version PSTELION, descried in this report, was successfully benchmarked over Brambilla's tokamak ICRF TORIC full wave code. The application of the code to stellarators is given in attached paper [16]. Calculations requiring a large number of Fourier harmonics generate very large matrices. While these can be written to disk (for some particular sparse matrix solvers) when necessary and subsequently retrieved as required, this increases the computer time substantially. The calculations on IBM-SP with 16 processors for 3D task problems for the NCSX plasma with NPSI $=71$, NTHETA $=64$, NZETA $=8$ has shown necessity about 1.3 GB of RAM and calculation time about 3 hours for CPU at $1.3 \mathrm{GHz}$. Doubling of poloidal or toroidal modes number leads to increase of CPU time about 5 times.

\section{Structure of PSTELION code}

PSTELION is constructed as a boundary value problem code. The notation and layout follow strictly to the standards and conventions of usual methodology to ensure that program is readily understood and easily transferred from one computer to another. Mnemonic variable names correspond closely to the notation used in this report and the program file contains indexes of all subroutines, COMMON blocks and variables used throughout the code. No extended library is required, the packet of subroutines for a complex matrix inversion is included.

The most effort is expended in initialization since the matrix of block bands diagonal system is calculated only once. Fig.1 shows the calling sequence in PSTELION. Equilibrium date is input, using the format of the VMEC code, in the WOUT file. This data is used to construct the metric tensor and other coefficients in METRIN. Splines technic is used to transfer this equilibrium metric from VMEC flux coordinate mesh ("radial") to fine flux mesh, required for a treatment of small scale Fast waves and smaller scale converted waves (in present version of PSTELION code - Ion Bernstein and Kinetic Alfven waves important for a treatment of "Heavy" minority ions scenario and "magnetic beach" scenario) in IC frequency range. This is accomplished in METRIC. Then metric elements and another coefficients are consequently replaced by their Fourier transforms by complex of Fast Fourier Transform subroutines FOURT1.

On a basis of these fine quantities the RF wave induced plasma currents, with account to the electron and ions Finite Larmor Radius effects, are calculated in subroutine IBWCURE. The kinetic tensor $\varepsilon_{\mathrm{ij}}$ is calculated in TENSOR block, and again they are consequently replaced by their Fourier transforms in FOURT2 block. To construct basic matrix, describing wave processes, are used 3 subroutines R_EQCOF, Y_EQCOF, Z_EQCOF, describing the coefficients in discretized ROT ROT E equations, and subroutines IBWCURE and TENSOR accumulating an information on metric, Jacobian, wave induced RF currents and kinetic tensor. Using these subroutines nonzero elements of the matrix are constructed in MLBLOCK.

Fourier transformed antenna currents are calculated in the block RIGHT (using subroutines PHD and FOURT1, FOURT2). To correctly describe toroidal component of the exciting currents some information on a metric is needed from METRICS. This provides a right hand side of a complex linear system $A x=b$. The resulting system of linear equations is solved in SOLVER by making use the ScaLAPACK library of parallelized linear algebra routines and direct use of the MPI interface. The found 
electrical fields, together with the wave induced currents or kinetic tensor from FOURT2, are used to calculate space RF power deposition profiles to the ions and electrons, as well as an antenna complex impedance ( needed for a matching with RF generator) and its toroidal and poloidal spectrums, in POWER. Output information and plotting are provided by OUT and GRAFIK subroutines.

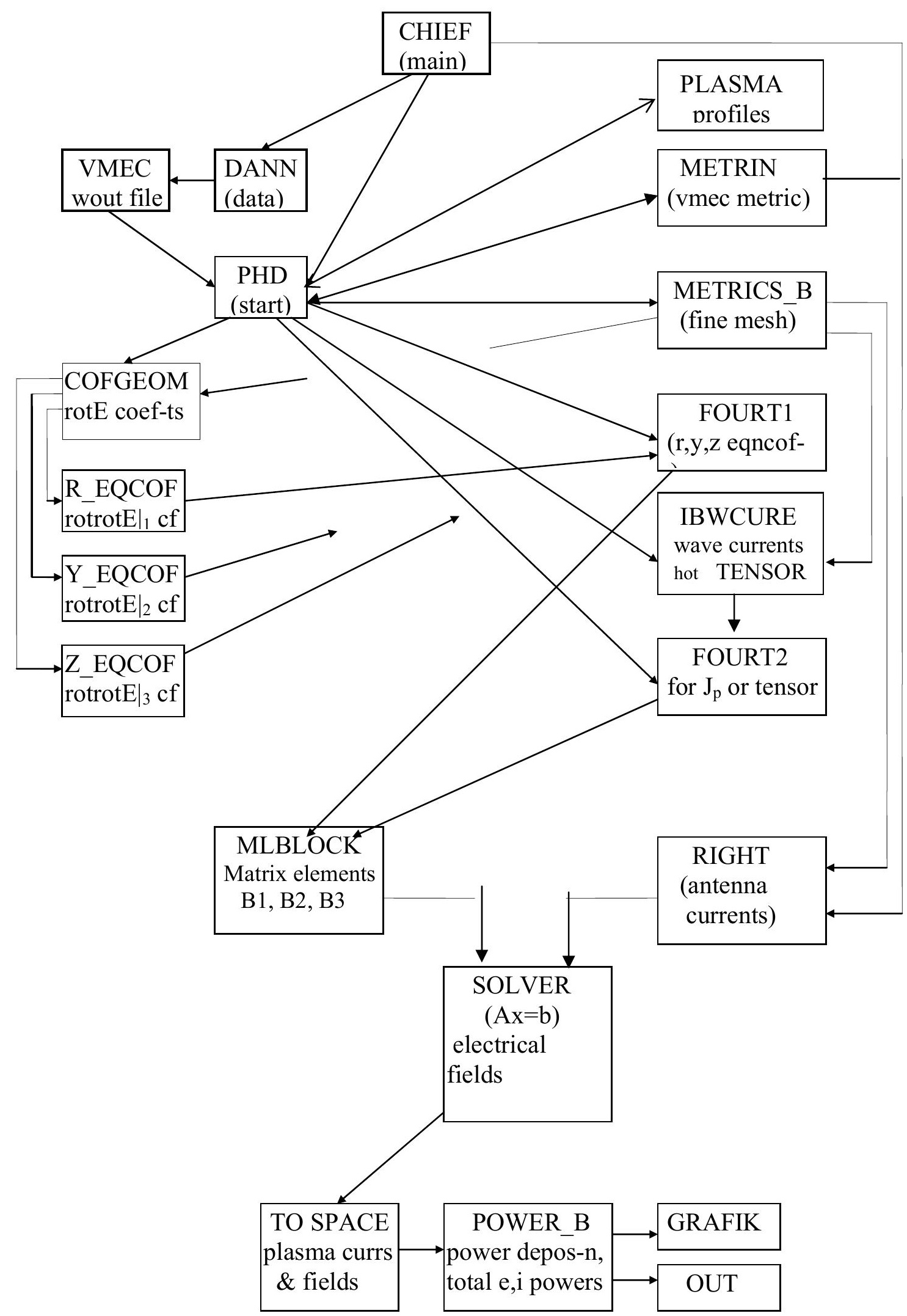




\subsection{Convergence study}

The ICRF heating/CD Mode Conversion (MC) scenarios treatment require large number of expansion Fourier terms. As an example we investigated $\mathrm{D}(\mathrm{He}-3)$ scenario in Alcator C-Mod tokamak [18] and D-T(Be-9) scenario in ITER [14]. First scenario operated at high central magnetic field $\mathrm{B}_{0}=7.85 \mathrm{~T}$ in deuterium plasma with $24 \%$ of Helium-3. For such high minority ions content more resolution is needed to resolve separation between the He-3 cyclotron layer near at the magnetic axis and the ion-ion hybrid layer at its left on the high field side. The Fig. 1 shows contour plots of wave radial electric fields abs $\left(\operatorname{real}\left(\mathrm{E}_{\psi}\right)\right)$ of a resolved mode conversion simulation for C-Mod on VMEC plasma equilibrium.

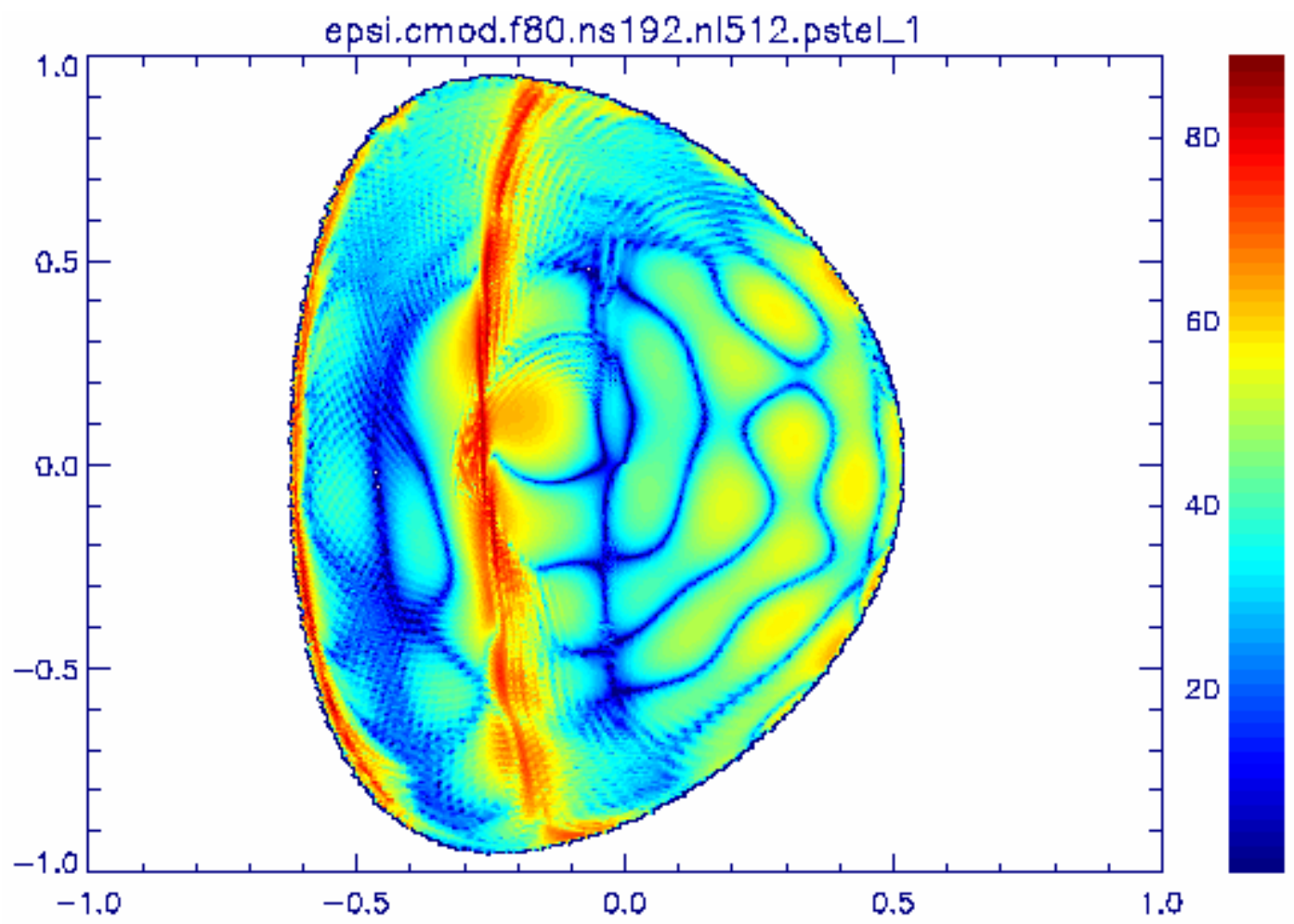

Fig, 1 Contour plots of abs(real $\left.\left(\mathrm{E}_{\psi}\right)\right)$ electric fields. The PSTELION simulation $\mathrm{D}(\mathrm{He}-3)$ MC scenario in C-Mod tokamak. $\mathrm{N}_{\mathrm{m}}=512, \mathrm{~N}_{\psi}=192$, $\mathrm{B}_{0}=7.85 \mathrm{~T}, \mathrm{f}=80 \mathrm{MHz}, \mathrm{n}_{\mathrm{He} 3} / \mathrm{n}_{\mathrm{e}}=0.24, \mathrm{n}_{\mathrm{e} 0}=210^{20} \mathrm{~m}^{-3}$. VMEC plasma equilibrium. 
One sees presence of the midplane IBW propagating to the left, the KAW above and below the midplane going to the right. The large scale Fast Waves propagate from outside antenna to the ion-ion hybrid layer, with small amount transmitting through. At high field side plasma boundary there is some power deposition to MC slow electrostatic waves at the Alfven resonance. At low poloidal modes number the excitation of the fields at the MC layer is not localised within the ion-ion hybrid layer but follows the flux surface geometry around toward the low field side to the He-3 resonance where it causes false He-3 power deposition. These results are similar to ones reported in [7] with the TORIC code.

In large scale ITER plasma the ICRF mode conversion scenarios, providing far offaxis RF driven currents, also require the large Fourier expansion modes to get a convergence. Much larger ion temperatures in ITER and lower magnetic field provide larger ion Larmor radii thereby reducing requirements on radial mesh size. Nevertheless, large number of poloidal modes again is needed. Example of mode converged MC driven current in D-T ITER plasma in steady state driven burn \#4 [14] is shown in Fig.2.

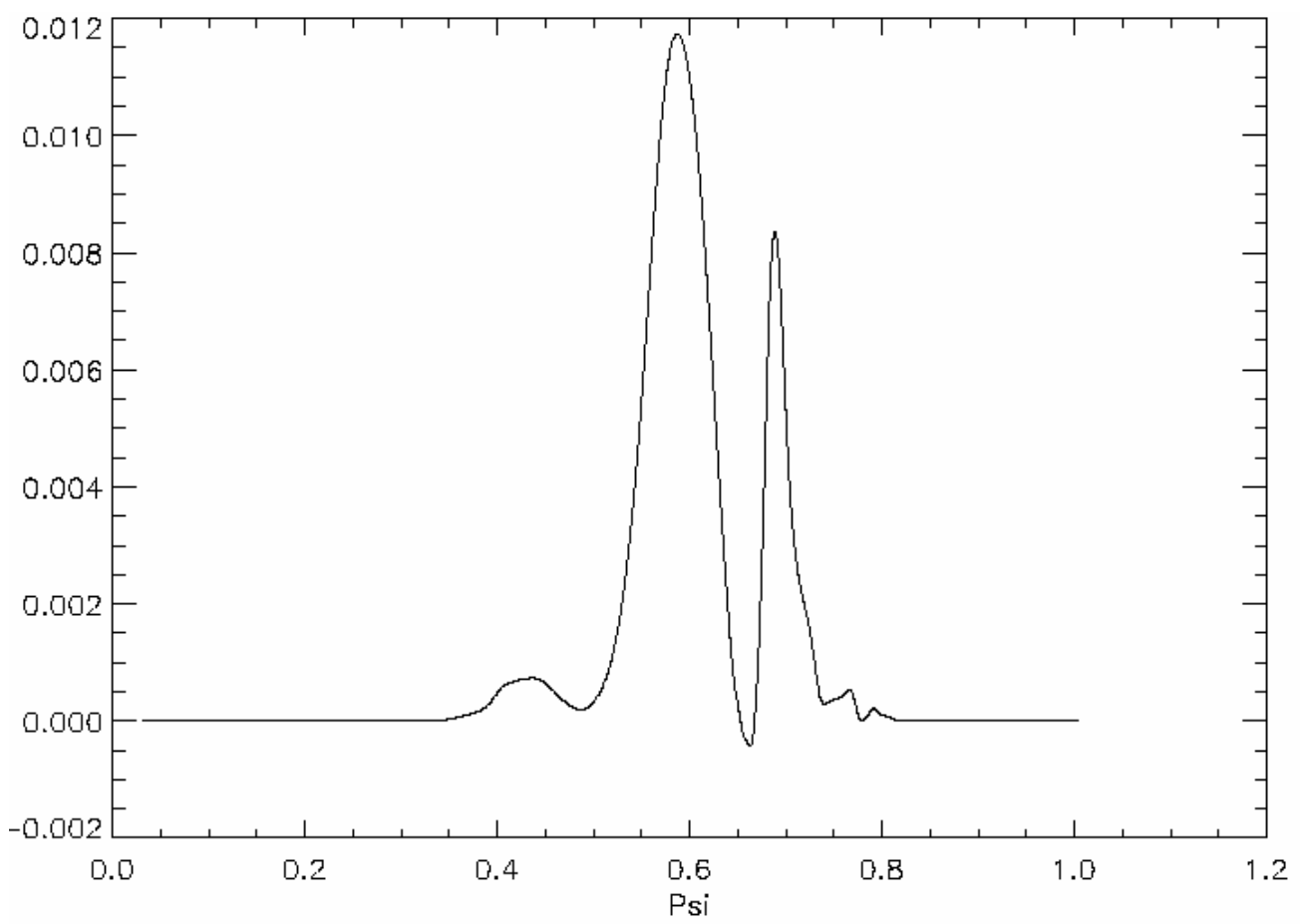

Fig,2 RF driven current radial profile (versus sqrt $(\rho), \rho=\sqrt{\Phi / \Phi_{0}}$ ) in the ITER. PSTELION simulation of a D+37\% T MC scenario.. Frequency $\mathrm{f}=30.8 \mathrm{MHz}, \mathrm{N}_{\mathrm{TOR}}=29, \mathrm{~N}_{\mathrm{m}}=512, \mathrm{~N}_{\psi}=490, \mathrm{~B}_{0}=5.3 \mathrm{~T}$, $\mathrm{n}_{\mathrm{e} 0}=110^{20} \mathrm{~m}^{-3}, \mathrm{~T}_{\mathrm{i} 0}=24 \mathrm{keV}$. VMEC high beta equilibrium.

Such convergence studies for stellarators have not done yet, due to large CPU time requirements. They are planned in future. 


\section{PROGRAM DESCRIPTION}

\subsection{Subprograms}

The majority of subprograms in PSTELION are listed below with brief summaries of their functions. This list is followed by more specific descriptions of the major subroutines in the code. All subroutines are written in the FORTRAN 77 and are in single precision. There is a version in FORTRAN 90 to accommodate dynamical massives.

DANN = initialization of certain numerical and physical input data

METRIN = reads input $3 \mathrm{D}$ (or 2D) equilibrium VMEC code Fourier harmonics WOUT

file data and does inverse transformation to real space metric tensor $g_{\mathrm{ij}}$, magnetic field BMOD, covariant and contravariant magnetic fields distributions, Jacobian, magnetic flux coordinate $s$ (PSI) and their "radial" (s) derivatives. Does transformation from VMEC radial mesh to more fine radial mesh by spline technic for plotting.

METRIB = calculation of plasma boundary, antenna and conducting shell boundaries.

METRICS_B $=$ calculation of equilibrium quantities on more fine radial, poloidal and toroidal $(s, \theta, \varphi)$ meshes and "semi" meshes needed for FFTN and wave calculations. Generation of PSI and "half" PSI meshes. Calculation of derivatives for metric tensor, Jacobian and contravariant magnetic field components, scaled to equilibrium magnetic field.

ELLIPS2 = calculations of above equilibrium quantities and their derivatives for elliptical 2D tokamak geometry for simple analyses and debugging.

GAMMAS_B $=$ calculation of free terms $\gamma_{\mathrm{ij}}$ in ROT E expressions. Their derivatives are calculated directly in calling subroutine PHD.

Calculation of terms

$\alpha_{\mathrm{ij}}$ in DIV_E and Grad_ $\Phi$ expressions Their derivatives are calculated directly in calling subroutine IBWCURE.

Calculation of coefficients $C_{2}, S_{2}$ in formula (4.2.6) involving reflection matrix R

COFGEOM $=$ calculation of $\varsigma_{\mathrm{ij}}$ coefficients in ROT E equations (section 2.1) and their derivatives.

COEF_EX = calculation of coefficients in psi component of ROT ROT E expression.

COEF_EY = calculation of coefficients in poloidal (THETA) component of ROTROT E expression.

COEF_EZ = calculation of coefficients in toroidal (PHI) component of ROT ROT E expression.

FFTN2B $=$ does Fast Fourier Transform of above ROT ROT E coefficients over poloidal THETA coordinate.

FFTN4B $=$ does Fast Fourier Transform of above ROT ROT E coefficients over toroidal PHI coordinate.

FFT_ANT $=$ does Analytical Fourier Transform of ICRF multi poloidal loop antenna 
over a toroidal coordinate.

IBWCURE $=$ does calculation of RF wave induced plasma currents, with account to the electron and ions Finite Larmor Radius effects, and their Fourier transforms through the subroutines SIGMAEX_FLR $\left(\mathrm{J}_{\mathrm{i}, \mathrm{x}}^{(2)}+\mathrm{J}_{\mathrm{x}}^{(\mathrm{elec})}\right)$,

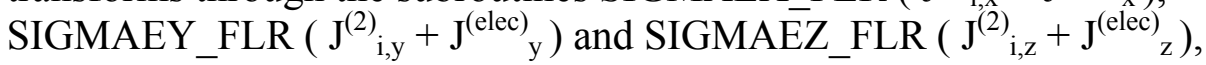

TENSOR = calculation of dielectric and conductivity kinetic tensors and their Fourier Transforms for the ORA code option. Calculation of roots of hot plasma local dispersion relation to treat finite Larmor radius corrections to the tensor.

The $1 \mathrm{D}$ evaluation of real and imaginary parts of perpendicular (to $\mathrm{B}_{0}$ ) wave vectors for debugging and to evaluate positions of cyclotron resonances of ion specious, cut off-s and singularities. The $1 \mathrm{D}$ evaluation of passed over plasma minor radius the Fast Wave RF power and optical thickness.

VMATRIX_B_EL = evaluation and non zero elements filling a basic linear problem matrix (to be inverted) with account to FLR effects for stellarators (3D problem, 3D open traps may be an option).

VMATRIX_RED2 = evaluation and non zero elements filling a basic linear problem matrix (to be inverted) with the ORA code option account to FLR effects for stellarators (3D problem, 3D open traps may be an option).

VMATRTOK_B_EL $=$ evaluation and non zero elements filling a basic linear problem matrix (to be inverted) with account to FLR effects for tokamaks (2D problem, 2D open traps may be an option).

VMATRTOK_RED2 = evaluation and non zero elements filling a basic linear problem matrix (to be inverted) with the ORA code option account to FLR effects for tokamaks (2D problem, 2D open traps may be an option).

ROT_REX $=$ calculation of the coefficients of ROT ROT E psi component. This information is needed for linear algebraic problem solver (working with real variables).

ROT_REY = calculation of the coefficients of ROT ROT E theta component. This information is needed for linear algebraic problem solver (working with real variables).

ROT_REZ = calculation of the coefficients of ROT ROT E phi component. This information is needed for linear algebraic problem solver (working with real variables).

SOLVE $=$ package of routines to solve linear system $\mathrm{Ax}=\mathrm{b}$ with complex matrix A and complex right hand side B. Uses parallelized linear algebra ScaLAPACK library routines and MPI Gaussian elimination and direct access write to disk and retrives information back when it is needed. This solver is especially useful for 3D huge matrix problems.

TOSPACE $=$ inverse FFTN of the wave fields to real space. 


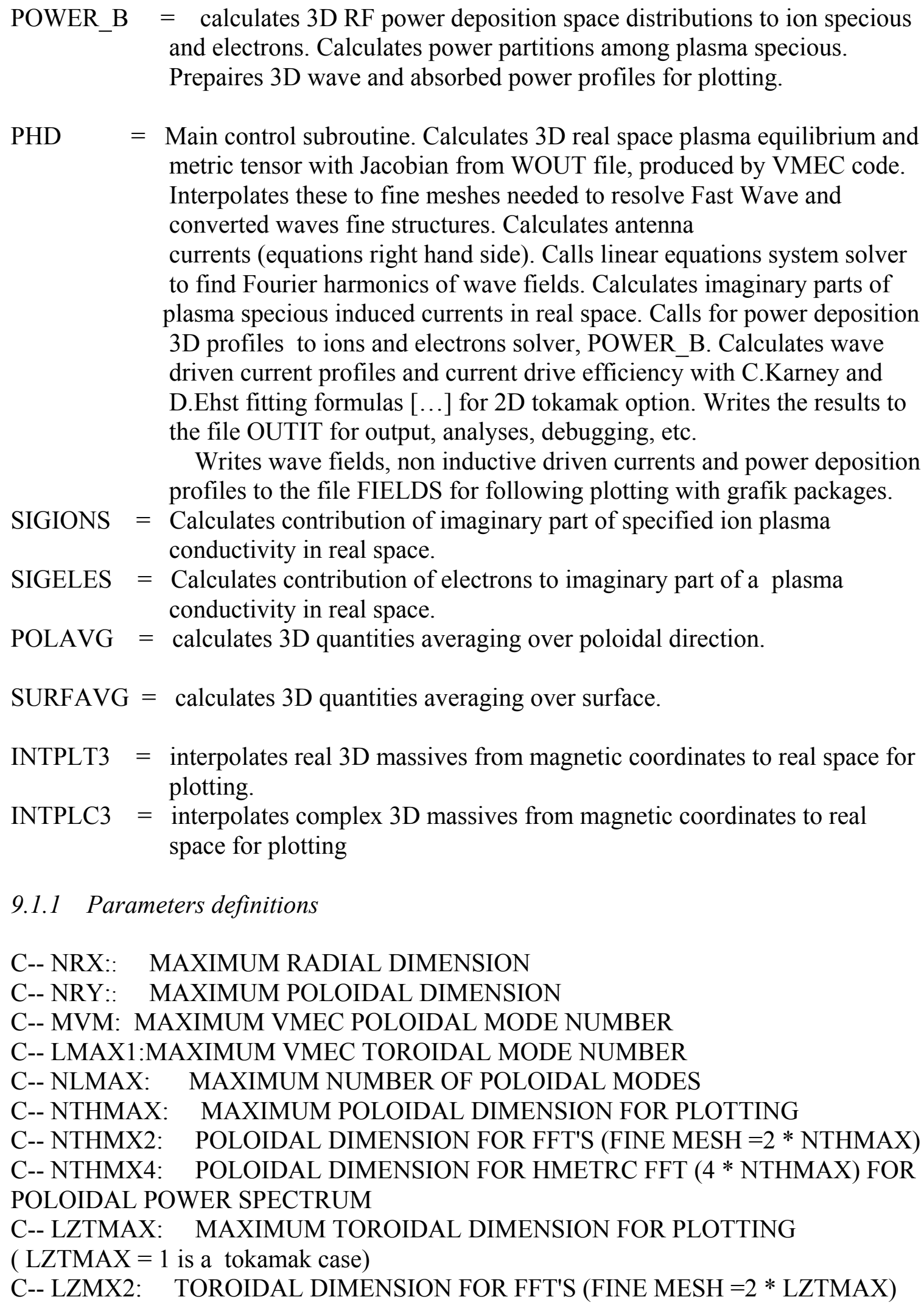
and electrons. Calculates power partitions among plasma specious. Prepaires 3D wave and absorbed power profiles for plotting.

PHD $=$ Main control subroutine. Calculates 3D real space plasma equilibrium and metric tensor with Jacobian from WOUT file, produced by VMEC code. Interpolates these to fine meshes needed to resolve Fast Wave and converted waves fine structures. Calculates antenna currents (equations right hand side). Calls linear equations system solver to find Fourier harmonics of wave fields. Calculates imaginary parts of plasma specious induced currents in real space. Calls for power deposition 3D profiles to ions and electrons solver, POWER_B. Calculates wave driven current profiles and current drive efficiency with C.Karney and D.Ehst fitting formulas [...] for 2D tokamak option. Writes the results to the file OUTIT for output, analyses, debugging, etc.

Writes wave fields, non inductive driven currents and power deposition profiles to the file FIELDS for following plotting with grafik packages.

SIGIONS $=$ Calculates contribution of imaginary part of specified ion plasma conductivity in real space.

SIGELES = Calculates contribution of electrons to imaginary part of a plasma conductivity in real space.

POLAVG $=$ calculates $3 \mathrm{D}$ quantities averaging over poloidal direction.

SURFAVG $=$ calculates $3 \mathrm{D}$ quantities averaging over surface.

INTPLT3 = interpolates real 3D massives from magnetic coordinates to real space for plotting.

INTPLC3 = interpolates complex 3D massives from magnetic coordinates to real space for plotting

\subsubsection{Parameters definitions}

C-- NRX:: $\quad$ MAXIMUM RADIAL DIMENSION

C-- NRY:: MAXIMUM POLOIDAL DIMENSION

C-- MVM: MAXIMUM VMEC POLOIDAL MODE NUMBER

C-- LMAX1:MAXIMUM VMEC TOROIDAL MODE NUMBER

C-- NLMAX: MAXIMUM NUMBER OF POLOIDAL MODES

C-- NTHMAX: MAXIMUM POLOIDAL DIMENSION FOR PLOTTING

C-- NTHMX2: $\quad$ POLOIDAL DIMENSION FOR FFT'S (FINE MESH $=2 *$ NTHMAX)

C-- NTHMX4: POLOIDAL DIMENSION FOR HMETRC FFT ( $4 *$ NTHMAX) FOR POLOIDAL POWER SPECTRUM

C-- LZTMAX: MAXIMUM TOROIDAL DIMENSION FOR PLOTTING

( $\mathrm{LZTMAX}=1$ is a tokamak case)

C-- LZMX2: $\quad$ TOROIDAL DIMENSION FOR FFT'S (FINE MESH $=2 *$ LZTMAX) 
CV- LZMX4: TOROIDAL DIMENSIOM FOR HMETRC FFT ( 4 * LZTMAX) FOR POWER SPECTRUM

C-- NZMAX: MAXIMUM NUMBER OF TOROIDAL MODES

(NZMAX $=1$ is a tokamak case)

C-- NLMAX3 $=3 *$ NLMAX $=$ THREE TIMES THE MAXIMUM NUMBER OF

POLOIDAL MODES

C-- MPMIN: MINIMUM POLOIDAL MODE NUMBER

C-- MPMAX: MAXIMUM POLOIDAL MODE NUMBER

C-- LZMAX: MAXIMUM TOROIDAL MODE NUMBER

C-- LZMIN: MINIMUM TOROIDAL MODE NUMBER

C-- MPMIN2: MINIMUM POLOIDAL MODE NUMBER FOR TWICE THE MODES (USED IN CONVOLUSIONS)

C-- LZMIN2: MINIMUM TOROIDAL MODE NUMBER FOR TWICE THE MODES (USED IN CONVOLUSIONS)

C-- MPMAX2: MAXIMUM POLOIDAL MODE NUMBER FOR TWICE THE MODES (USED IN CONVOLUSIONS)

C-- LZMAX2: MAXIMUM TOROIDAL MODE NUMBER FOR TWICE THE MODES (USED IN CONVOLUSIONS)

C-- MPMIN4: MINIMUM POLOIDAL MODE NUMBER FOR 4*MODES (USED IN CONVOLUSIONS)

C-- MPMAX4: MAXIMUM POLOIDAL MODE NUMBER FOR 4*MODES (USED IN CONVOLUSIONS)

C-- LZMIN4: MINIMUM TOROIDAL MODE NUMBER FOR 4*MODES (USED IN CONVOLUSIONS)

C-- LZMAX4: MAXIMUM TOROIDAL MODE NUMBER FOR 4*MODES (USED IN CONVOLUSIONS)

C-- LZFAM1: MINIMUM FAMILY'S TOROIDAL MODE NUMBER

C-- LZFAM2: MAXIMUM FAMILY'S TOROIDAL MODE NUMBER

$\mathrm{C}-$ NSPEC MAXIMUM OF PLASMA ION SPECIOUS

\subsubsection{Subroutine DANN}

Purpose Initializes certain numerical and physical input data.

Use The calling sequence is CALL DANN.

The major information produced by DANN is in common blocks $/ /, / /, \ldots$ which are described below.

Table 1

Content of common block /MAGFLD/ NFP:

Input variable Meaning Units

(FORTRAN name)

NFP - NUMBER OF CONFINING MAGNETIC FIELD PERIODS

NFP LATER ALSO COMES from READING WOUT FILE IN s-ne METRIN

$\mathrm{C} \mathrm{NFP}=1$ for tokamak 
Table 2

Contents of common block STELL

COMMON/STELL/MPOL1,MPOL2,LTOR1,LTOR2,NL,LZ,NTHETA,LZETA, $1 \quad$ NTHET2,LZET2,NTHET4,LZET4,IANT,EPS0,AMU0

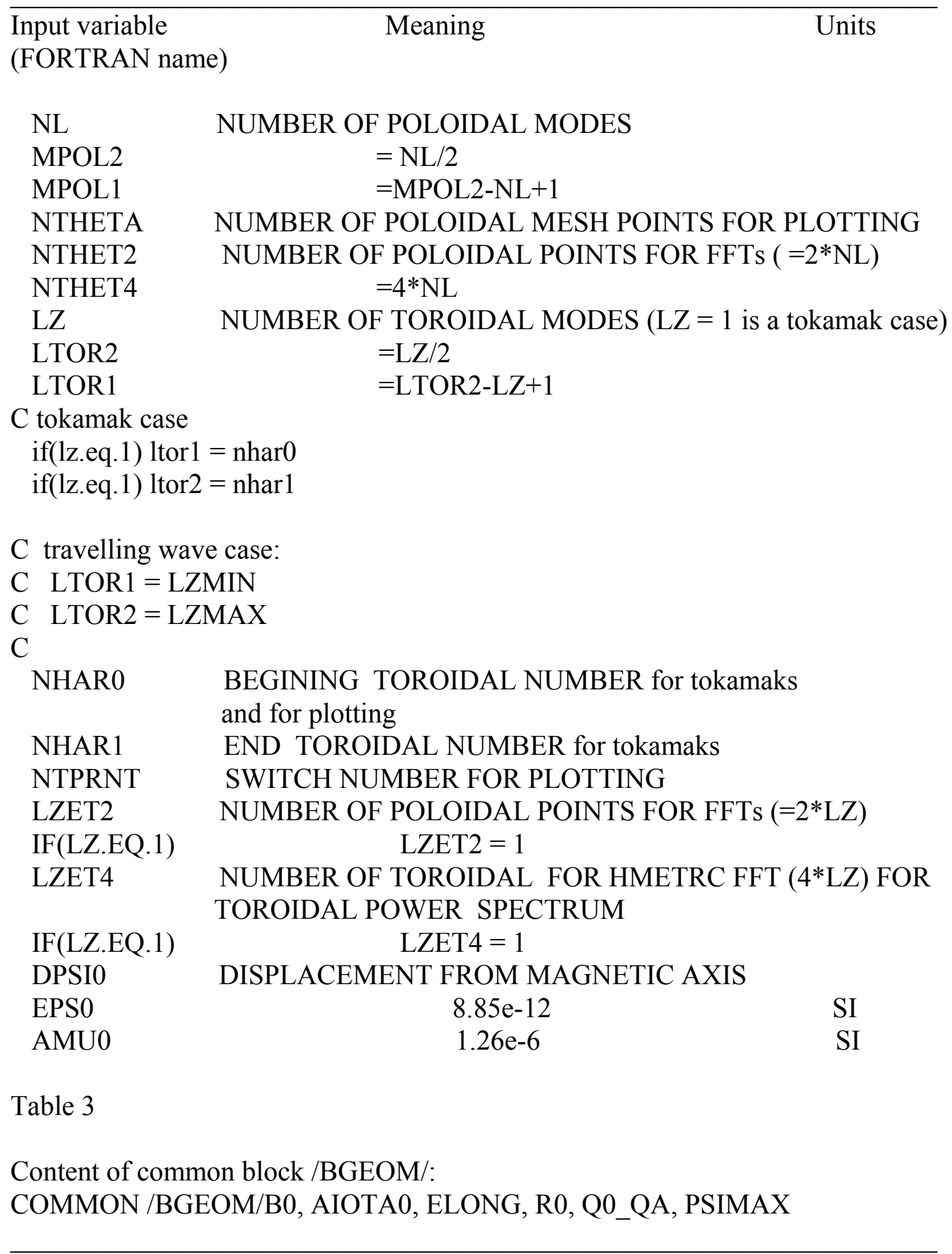


Input variable

(FORTRAN name)

B0 TOROIDAL MAGNETIC FIELD AT MAGNETIC AXIS, T

AIOTA0 ROTATIONAL TRANSFORM AT MAGNETIC AXIS $(=1 / \mathrm{Q} 0)$

ELONG PLASMA ELONGATION

R0 PLASMA MAJOR RADIUS AT MAGNETIC AXIS, m

Q0_QA SAFETY FACTORS RATIO Q0/QA

PSIMAX MAXIMUM DIMENSIONAL RADIAL COORDINATE

Table 4

Content of common block /GEOM/:

COMMON /GEOM/ IGEOM

\begin{tabular}{cll}
\hline $\begin{array}{l}\text { Input variable } \\
\text { (FORTRAN name) }\end{array}$ & \multicolumn{1}{c}{ Meaning } \\
C & \multicolumn{1}{c}{ UGEOM: } & plasma geometry: \\
IGEOM $=1$ & ELLIPTICAL PLASMA (tokamak case) \\
IGEOM $=2$ & VMEC's PLASMA EQUILIBRIUM (3D case) \\
IGEOM $=3$ & SOLOV'EV PLASMA EQUILIBRIUM (tokamak case) \\
IGEOM $=4$ & D-shaped pseudo PLASMA EQUILIBRIUM (tokamak case)
\end{tabular}

Table 5

Content of common block /METR1/:

COMMON/METR1/ BET_N,XWALL,IANTEN,IEZ,DPSI0,SCRAPE

\begin{tabular}{llc}
\hline Input variable & Meaning & Units \\
(FORTRAN name) & &
\end{tabular}

\begin{tabular}{|c|c|}
\hline BET_N & EXPONENT IN: $\mathrm{N}=(\mathrm{N} 0-\mathrm{NLIM}) *\left(1-\mathrm{RHO}^{\wedge} \mathrm{BET} / \mathrm{N}\right)^{\wedge} \mathrm{ALF}+\mathrm{N}+\mathrm{NLIM}$ \\
\hline XWĀLL & $\begin{array}{l}\text { CONDUCTING WALL COORDINATE AT EQUUATORIAL } \\
\text { PLANE }\end{array}$ \\
\hline IANTEN $=0$ & STEP FUNCTION ANTENNA IN POLOIDAL COORDINATE \\
\hline IANTEN $=1$ & GAUSSIAN ANTENNA IN POLOIDAL COORDINATE \\
\hline IEZ & $\begin{array}{l}\text { SWITCH FOR E PARALLEL CALCULATION MODE } \\
\text { IF(IEZ.NE.0.AND.IEZ.NE.-1) THEN E PARALLEL } \\
\text { IS CALCULATED FROM COMPLETE EQUATION } \\
\text { IF(IEZ.EQ.0) E PARALLEL IS SET TO ZERO IN SOLUTION } \\
\text { IF(IEZ.EQ.-1) E PARALLEL IS SET TO ZERO AFTER } \\
\text { SOLUTION }\end{array}$ \\
\hline PSI0 & MESH DISPLACEMENT FROM MAGNETIC AXIS \\
\hline
\end{tabular}


SCRAPE SCRAPE IS DIVERTOR LAYER THICNESS EXPONENT,

Table 6 in METERS

Content of common block /ANTSTE/:

COMMON/ANTSTE/ DELTAP,P,DELTZ,PZET,WIDTH,WIDTHZ

\begin{tabular}{ll}
\hline $\begin{array}{l}\text { Input variable } \\
\text { (FORTRAN name) }\end{array}$ & \multicolumn{1}{c}{ Meaning } \\
& \\
DELTA_P & POLOIDAL WIDTH OF THE ANTENNA IN UNITS OF PI \\
P & POLOIDAL POSITION OF ANTENNA IN UNITS OF PI \\
DELTA_Z & TOROIDAL WIDTH OF THE ANTENNA IN UNITS OF PI \\
PZET & TOROIDAL POSITION OF ANTENNA IN UNITS OF PI \\
WIDTH & POLOIDAL WIDTH OF STEP FUNCTION ANTENNA IN \\
& UNITS OF PI \\
WIDTHZ & TOROIDAL WIDTH OF STEP FUNCTION ANTENNA IN \\
& UNITS OF PI
\end{tabular}

Table 7

Content of common block /ANTCOM/:

COMMON/ANTCOM/ THETAC,ZETAC

\begin{tabular}{ll}
\hline $\begin{array}{l}\text { Input variable } \\
\text { (FORTRAN name) }\end{array}$ & Meaning \\
& \\
THETA_C & POLOIDAL ANTENNA LOCATION IN UNITS OF PI \\
ZETA_C & TOROIDAL ANTENNA LOCATION IN UNITS OF PI
\end{tabular}

Table 8

Content of common block /DAT/:

COMMON/DAT/ ATOR,BTOR,CTOR,RTOR,RNE0,TE0,SUMJ, OMG

\begin{tabular}{lll}
\hline $\begin{array}{l}\text { Input variable } \\
\text { (FORTRAN name) }\end{array}$ & \multicolumn{1}{c}{ Meaning } & Units \\
& & \\
ATOR & PLASMA RADIUS IN EQUATORIAL PLANE, & $\mathrm{M}$ \\
BTOR & ANTENNA RADIUS IN EQUATORIAL PLANE, & $\mathrm{M}$ \\
CTOR & WALL RADIUS IN EQUATORIAL PLANE, & $\mathrm{M}$ \\
RTOR & TOR MAJOR RADIUS, & $\mathrm{M}$ \\
RNE0 & ELECTRON DENSITY AT MAGNETIC AXIS, & $\mathrm{M}-3$
\end{tabular}


TE0

SUMJ

OMG
ELECTRON TEMPERATURE AT MAGNETIC AXIS, eV ANTENNA LOOP CURRENT,

ANGULAR WAVE FREQUENCY,
AMPER

HERTZ

Table 9

Content of common block /JAK $1 /$ :

COMMON/JAK1/ ALF_N,ALF_TE,ALF_TI,BET_TE,BET_TI,ANLIM,PSIB,PSIW, TILIM,TELIM

\begin{tabular}{|c|c|}
\hline $\begin{array}{l}\text { Input variable } \\
\text { (FORTRAN name) }\end{array}$ & Meaning \\
\hline ALF_N & $\begin{array}{l}\text { POWER TO WHICH THE PARABOLIC ELECTRON } \\
\text { DENSITY IS RAISED }\end{array}$ \\
\hline ALF_TI & $\begin{array}{l}\text { POWER TO WHICH THE PARABOLIC ION TEMPERATURE } \\
\text { IS RAISED }\end{array}$ \\
\hline ALF_TE & $\begin{array}{l}\text { POWER TO WHICH THE PARABOLIC ELECTRON } \\
\text { TEMPERATURE IS RAISED }\end{array}$ \\
\hline BET_TE & $\begin{array}{l}\text { EXPONENT IN: TE }=(\text { TE0-TELIM })^{*}\left(1-\mathrm{RHO}^{\wedge} \mathrm{BET} \_\mathrm{TE}\right)^{\wedge} \mathrm{ALF} \_\mathrm{TE} \\
+ \text { TELIM }\end{array}$ \\
\hline BET_TI & $\begin{array}{l}\text { EXPONENT IN: TI=(TI0-TILIM })^{*}\left(1-\mathrm{RHO}^{\wedge} \mathrm{BET}_{-} \mathrm{TI}\right)^{\wedge} \mathrm{ALF}-\mathrm{TI}+ \\
\text { TILIM }\end{array}$ \\
\hline ANLIM & ELECTRON DENSITY IN SCRAPE-OFF REGION (X>APLASM) \\
\hline PSIB & NORMALISED POLOIDAL FLUX AT CONDUCTING SHELL \\
\hline PSIA & NORMALISED POLOIDAL FLUX AT PLASMA BOUNDARY \\
\hline TILIM & ION TEMPERATURE AT A LIMITER (SEPARATRIS) \\
\hline TELIM & ELECTRON TEMPERATURE AT A LIMITER (SEPARATRIS) \\
\hline
\end{tabular}

Table 10

Content of common block/DENCOM/:

COMMON/DENCOM/DLTPSI, ANWALL, ANPED

\begin{tabular}{ll}
\hline $\begin{array}{l}\text { Input variable } \\
\text { (FORTRAN name) }\end{array}$ & Meaning \\
& \\
DLTPSI & CHARACTERISTIC PSI SCALE IN SCRAPE OFF LAYER \\
ANWALL & PLASMA DENSITY AT CONDUCTING WALL \\
ANPED & MINIMUM ELECTRON DENSITY ALLOWABLE AT
\end{tabular}




\section{PEDESTAL}

Table 11

Content of common block /TEMPE/:

COMMON/TEMPE/ TI01,TI02,TI03,TI04,TI05,TI06,TI07,TI08, TILIM,TELIM

\begin{tabular}{ll}
\hline $\begin{array}{l}\text { Input variable } \\
\text { (FORTRAN name) }\end{array}$ & Meaning \\
& \\
TI01,...,TI08 & CENTRAL VALUES OF ION TEMPERATURE of specios 1-8 IN eV \\
TILIM & ION TEMPERATURE IN SCRAPE-OFF REGION (X>APLASM) \\
TELIM & ELECTRON TEMPERATURE IN SCRAPE-OFF REGION \\
& $(\mathrm{X}>$ APLASM)
\end{tabular}

Table 12

Content of common block /COMPO/:

COMMON /COMPO / ZI(NCO),AI(NCO),PR(NCO)

\begin{tabular}{llc}
\hline $\begin{array}{l}\text { Input variable } \\
\text { (FORTRAN name) }\end{array}$ & \multicolumn{1}{c}{ Meaning } \\
ZI & ARRAY OF ELECTRICAL CHARGES OF PLASMA SPECIOS \\
& (ELECTRONS AND IONS) \\
AI & ARRAY OF PLASMA PARTICLES MASSES (IN PROTON MASS) \\
PR & ARRAY OF PARTITIONS OF PLASMA SPECIOS MESUARED \\
& IN ELECTRON DENSITY \\
NCO & MAXIMUM NUMBER OF PLASMA SPECIOS (ELECTRONS AND \\
& IONS)
\end{tabular}

Table 13

Content of common block /DANN/:

COMMON/DANN/ NCOIL,QCENTR,PHASE,D_LOOP

\begin{tabular}{llc}
\hline $\begin{array}{l}\text { Input variable } \\
\text { (FORTRAN name) }\end{array}$ & Mnits \\
NCOIL & NUMBER OF ANTENNA COILS \\
QCENTR & SAFETY FACTOR AT MAGNETIC AXIS \\
PHASE & FEEDING PHASE DISPLACEMENT BETWEEN ADJACENT COILS \\
D_LOOP & TOROIDAL WIDTH OF ANTENNA INDIVIDUAL COIL
\end{tabular}

9.1.3 Subroutine PHD 


\section{Purpose:}

Calculates 3D real space plasma equilibrium and metric tensor with Jacobian from WOUT file, produced by VMEC code. Interpolates these to fine meshes needed to resolve Fast Wave and converted waves fine structures. Calculates ROT ROT E expressions, tensor contributions and their Fast Fourier Transfoms. Calculates antenna currents (equations right hand side). Calls linear equations system solver to find Fourier harmonics of wave fields. Calculates imaginary parts of plasma specious induced currents in real space. Calls for power deposition 3D profiles to ions and electrons solver, POWER. Calculates wave driven current profiles and current drive efficiency with famous C.Karney and D.Ehst fitting formulas for 2D tokamak option. Writes the results to the file OUTIT for output, analyses, debugging, etc. Writes wave fields, non inductive driven currents and power deposition profiles to the file FIELDS for following plotting with grafik packages.

Flags:

C---TOKAMAK Logical variable - plasma configuration choice $=$.true. - tokamak choice. Code operates in $2 \mathrm{D}$ version

$=$.false $-3 \mathrm{D}$ code version (e.g. stellarator)

C---IANTANAL Antenna toroidal wave spectrum model choice flag

$=1$ - analytical model (for more accurate excitation spectrum calculation - in conditions of limited number of toroidal harmonics for a plasma)

$=0$ - regular FFT approach in toroidal (zeta) direction

C-IFLR ION FINITE LARMOR RADIUS CONTRIBUTIONS FLAG

$=1$ included

$=0$ ignored (order reduction algorithm)

C-IFLRE ELECTRON FINITE LARMOR RADIUS CONTRIBUTIONS FLAG

$=1$ included ( TTMP and CROSS terms)

$=0$ ignored (zero Larmor radius ELD and ORA electron corrections to the dielectric tensor )

C--IBWELD Electron Landau damping of Ion Bernstein waves (if IFLR = 1)

$\mathrm{C} \quad=0$ ignored

C $=1 \delta \sigma^{(2)}$ addition from the quasi-electrostatic dispersion relation

Use: The calling sequence is CALL PHD.

The major input information is supplied by subroutine DANN through its common blocks, described above, and through the WOUT file produced by VMEC code.

Table 2 
List of major arrays in subroutine PHD

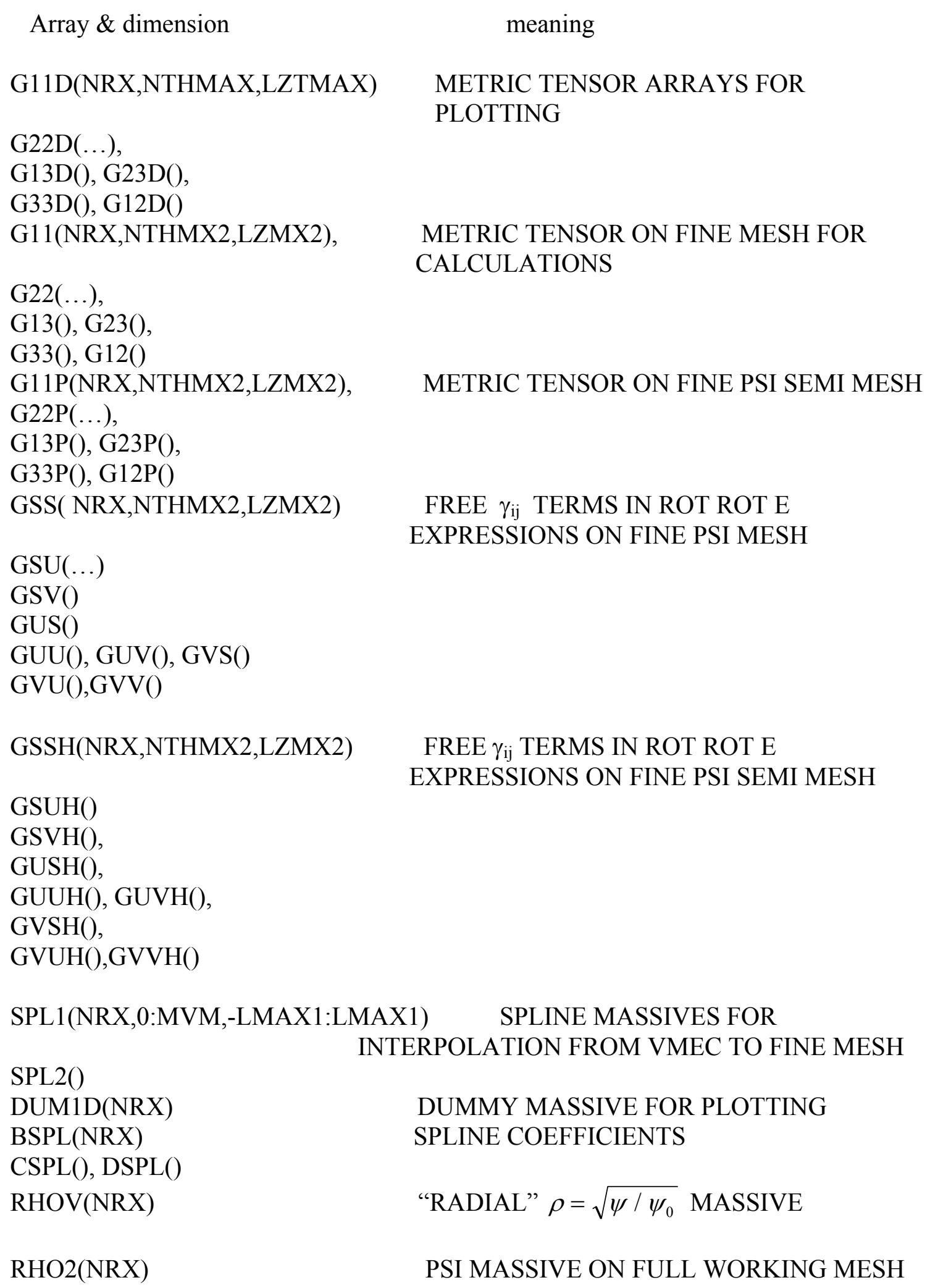

Array \& dimension

G11D(NRX,NTHMAX,LZTMAX)

$\mathrm{G} 22 \mathrm{D}(\ldots)$,

G13D(), G23D(),

G33D(), G12D()

G11(NRX,NTHMX2,LZMX2),

G22(...),

G13(), G23(),

G33(), G12()

G11P(NRX,NTHMX2,LZMX2),

$\mathrm{G} 22 \mathrm{P}(\ldots)$,

G13P(), G23P(),

G33P(), G12P()

GSS( NRX,NTHMX2,LZMX2)

GSU( ...)

GSV()

GUS()

GUU(), GUV(), GVS()

GVU(),GVV()

GSSH(NRX,NTHMX2,LZMX2)

GSUH()

GSVH(),

GUSH(),

GUUH(), GUVH(),

GVSH(),

GVUH(),GVVH()

SPL1(NRX,0:MVM,-LMAX1:LMAX1) SPLINE MASSIVES FOR

INTERPOLATION FROM VMEC TO FINE MESH

SPL2()

DUM1D(NRX)

BSPL(NRX)

CSPL(), DSPL()

RHOV(NRX)

RHO2(NRX)

meaning

METRIC TENSOR ARRAYS FOR

PLOTTING

METRIC TENSOR ON FINE MESH FOR CALCULATIONS

METRIC TENSOR ON FINE PSI SEMI MESH

FREE $\gamma_{\mathrm{ij}}$ TERMS IN ROT ROT E EXPRESSIONS ON FINE PSI MESH

FREE $\gamma_{\mathrm{ij}}$ TERMS IN ROT ROT E EXPRESSIONS ON FINE PSI SEMI MESH 


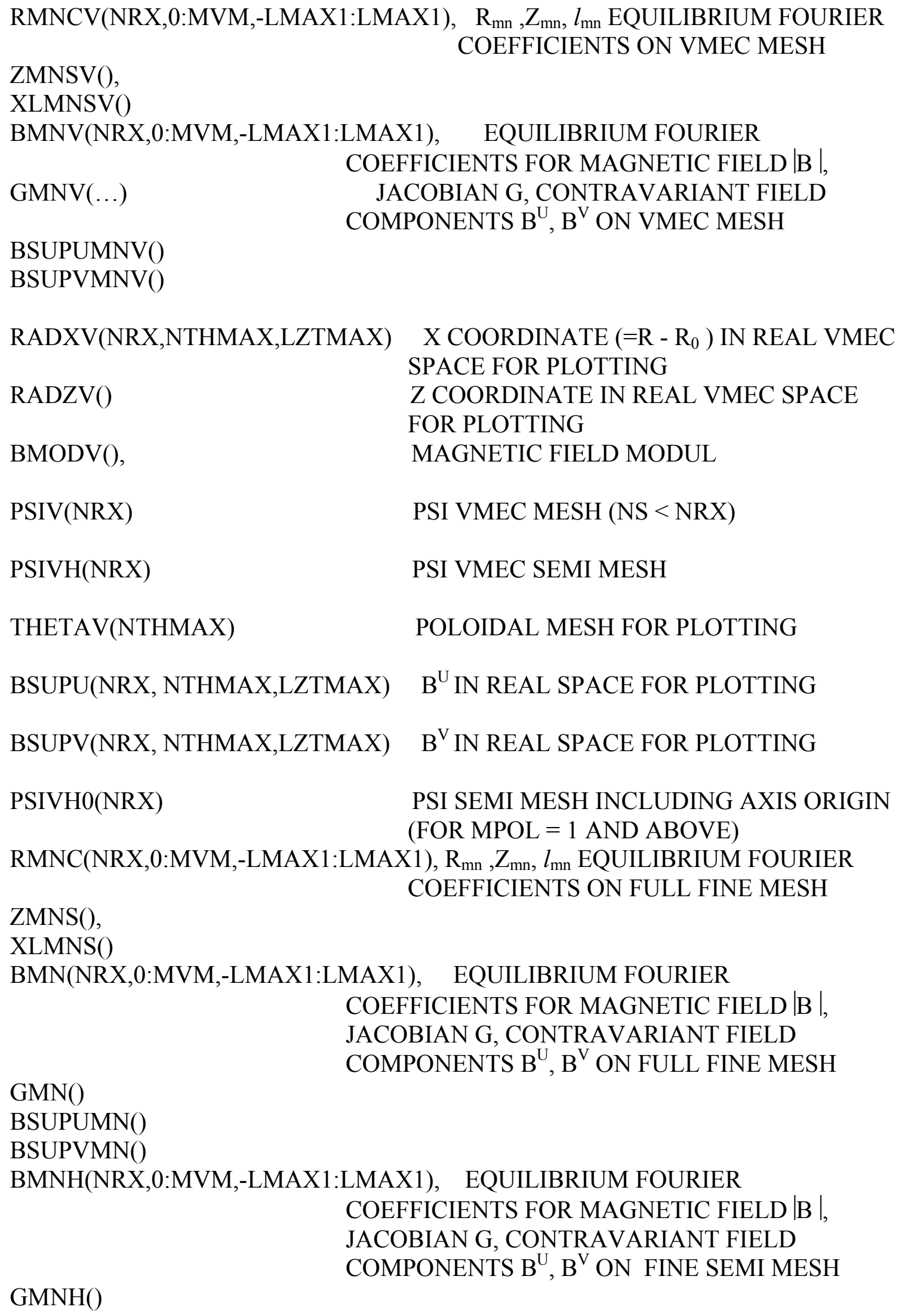




$$
\begin{aligned}
& \text { BSUPUMNH() } \\
& \text { BSUPVMNH() } \\
& \text { DRMNC(NRX,0:MVM,-LMAX1:LMAX1) } \\
& \text { DZMNS() } \\
& \operatorname{DBMN}() \\
& \text { D2RMNC() } \\
& \text { D2ZMNS() } \\
& \text { DBSUPU()) } \\
& \text { DBSUPV() } \\
& \text { RADRV(NRX,NTHMAX,LZTMAX), } \\
& \text { THESTR() } \\
& \text { DRDRHOV(NRX,NTHMAX, LZTMAX) } \\
& \text { DZDRHOV() } \\
& \text { DRDTHV() } \\
& \text { DZDTHV() } \\
& \text { DBDRHOV() } \\
& \text { DBDTHV() } \\
& \text { ZETA(LZTMAX) } \\
& \text { ZESTR(NRX,NTHMAX,LZTMAX) } \\
& \text { DZDZETV(NRX,NTHMAX,LZTMAX) } \\
& \text { TOROIDAL PHI ANGLE MESH FOR } \\
& \text { PLOTTING } \\
& \text { ZETA STREAM FUNCTION } \\
& \text { NEEDED FOR PLOTTING } \\
& \text { THETA STREAM FUNCTION } \\
& \text { NEEDED FOR PLOTTING } \\
& \frac{\partial R}{\partial \rho} \text { on VMEC space mesh } \\
& \frac{\partial Z}{\partial \rho} \text { on VMEC mesh } \\
& \frac{\partial R}{\partial \theta} \quad \text { on VMEC mesh } \\
& \frac{\partial R}{\partial \theta} \quad \text { on VMEC mesh } \\
& \frac{\partial B}{\partial \rho} \text { on VMEC mesh } \\
& \frac{\partial B}{\partial \theta} \text { on VMEC mesh } \\
& \frac{\partial R_{m n}}{\partial \psi} \\
& \frac{\partial Z_{m n}}{\partial \psi} \\
& \frac{\partial B_{m n}}{\partial \psi} \\
& \frac{\partial^{2} R_{m n}}{\partial \psi^{2}} \\
& \frac{\partial^{2} Z_{m n}}{\partial \psi^{2}} \\
& \frac{\partial B^{u}{ }{ }}{\partial \psi} \\
& \frac{\partial B^{V}{ }_{m n}}{\partial \psi} \\
& \mathrm{R}(\psi, \theta, \varphi) \text { from VMEC } \\
& \frac{\partial Z}{\partial \varphi} \text { on VMEC mesh }
\end{aligned}
$$


DRDZETV()

ZETAV(LZTMAX)

THGRD(NTHMAX)

THGRD2(NTHMX2)

PSIN(NRX)

PSINP(NRX)

ZETA2(LZMX2)

THET4(NTHMX4),

FTHETA(NTHMAX)

FTHET2(NTHMX2)

FZETA(LZTMAX)

FZET2(LZMX2) $\frac{\partial R}{\partial \varphi}$ on VMEC mesh

PHI TOROIDAL ANGLE on VMEC mesh

POLOIDAL ANGLE WORKING MESH

FOR PLOTTING

FINE POLOIDAL ANGLE WORKING

MESH FOR PLOTTING

"RADIAL" PSI MESH

“RADIAL” PSI SEMI MESH

FINE TOROIDAL ANGLE MESH

TWICE FINE POLOIDAL MESH

WORKING ANTENNA ARRAY FOR PLOTTING

FINE WORKING ANTENNA ARRAY

WORKING ANTENNA TOROIDAL ARRAY FOR PLOTTING

FINE WORKING ANTENNA TOROIDAL ARRAY

BSPRMN(NRX,0:MVM,-LMAX1:LMAX1), SPLINE MASSIVES FOR INTERPOLATION $\mathrm{R}_{\mathrm{mn}}$ FROM VMEC

RADIAL MESH TO WORKING ONE

CSPRMN()

$\operatorname{DSPRMN}()$

BSPZMN(NRX,0:MVM,-LMAX1:LMAX1) SPLINE MASSIVES FOR INTERPOLATION $Z_{\mathrm{mn}}$ FROM VMEC RADIAL MESH TO WORKING ONE

$\operatorname{CSPZMN}()$

DSPZMN()

BSPBMN(NRX,0:MVM,-LMAX1:LMAX1) SPLINE MASSIVES FOR INTERPOLATION $|\mathrm{B}|_{\mathrm{mn}}$ FROM VMEC RADIAL MESH TO WORKING ONE

$\operatorname{CSPBMN}()$

$\operatorname{DSPBMN}()$

BSPBUMN(NRX,0:MVM,-LMAX1:LMAX1) SPLINE MASSIVES FOR 


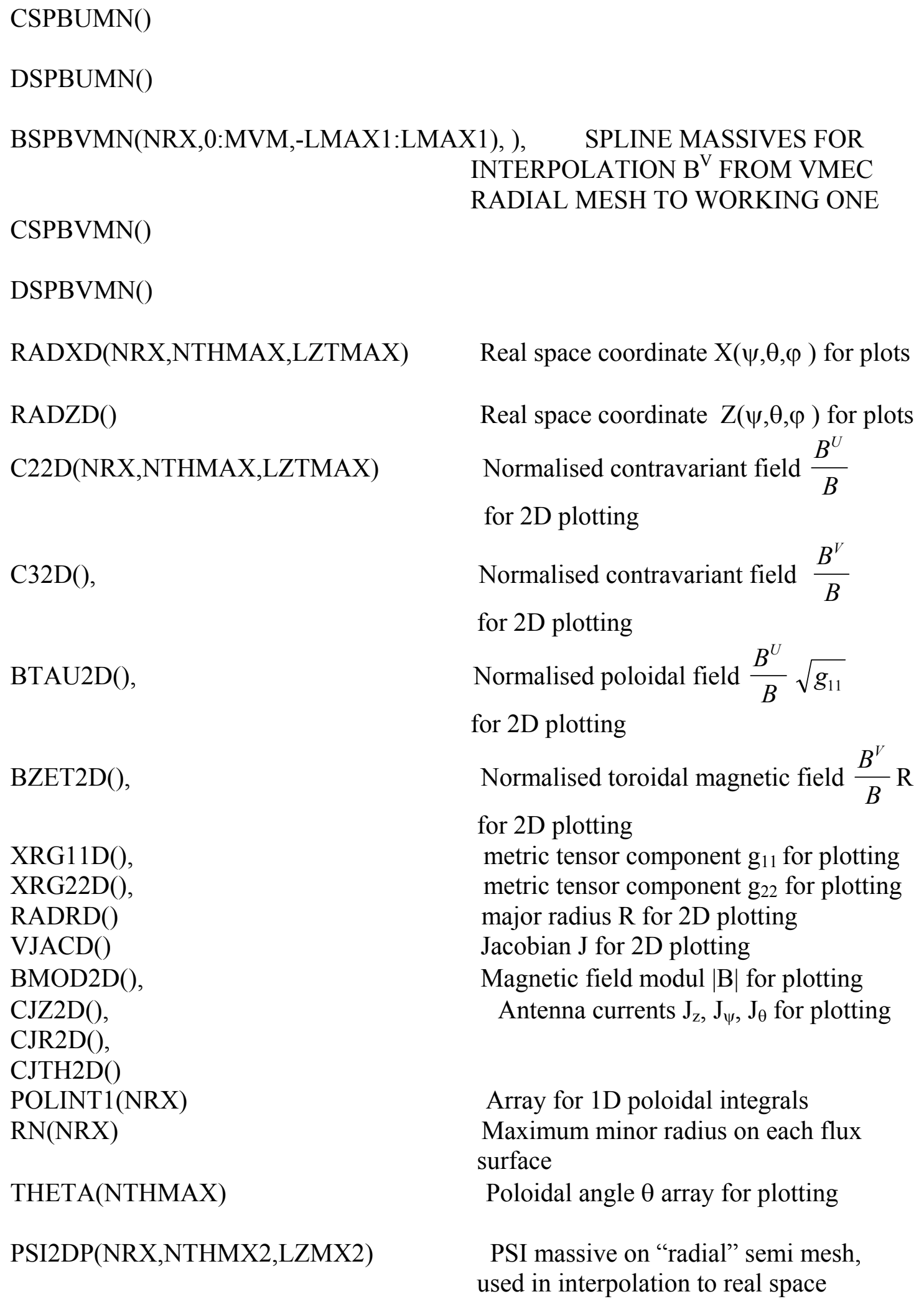




\begin{tabular}{|c|c|}
\hline THE2DP() & $\begin{array}{l}\text { theta massive on "radial" semi mesh, } \\
\text { used in interpolation to real space }\end{array}$ \\
\hline BMODP(), & Module of magnetic field on semi mesh \\
\hline BPLOT() & Module of magnetic field for plotting \\
\hline PSI2D(NRX,NTHMAX,LZTMAX) & $\begin{array}{l}\text { PSI massive on "radial" mesh, used } \\
\text { in interpolation to real space for } 2 \mathrm{D} \text { plotting }\end{array}$ \\
\hline THE2D(NRX,NTHMAX,LZTMAX), & $\begin{array}{l}\text { theta massive on "radial" mesh, used } \\
\text { in interpolation to real space for } 2 \mathrm{D} \text { plotting }\end{array}$ \\
\hline $\mathrm{B} 2 \mathrm{D}()$ & Module of magnetic field for 2D plotting \\
\hline RADX(NRX,NTHMX2,LZMX2), & $\begin{array}{l}\text { Real space coordinate } \mathrm{X}(\psi, \theta, \varphi) \text { for } \\
\text { calculations }\end{array}$ \\
\hline RADZ(), & $\begin{array}{l}\text { Real space coordinate } Z(\psi, \theta, \varphi) \text { for } \\
\text { calculations }\end{array}$ \\
\hline $\begin{array}{l}\text { XG12(NRX,NTHMX2,LZMX2), } \\
\text { XRG11(), }\end{array}$ & $\begin{array}{l}\text { Dummy metric tensor } \mathrm{g}_{12} \text { component } \\
\qquad \sqrt{g_{11}}\end{array}$ \\
\hline $\begin{array}{l}\text { VJAC() } \\
\text { BT_NT0(NTHMX2,LZMX2), } \\
\text { BT NTP(), }\end{array}$ & $\begin{array}{l}\text { Jacobian } \mathrm{J} \text { on fine mesh } \\
\mathrm{B}^{\mathrm{U}} \\
\mathrm{B}^{\mathrm{U}} \text { on semi mesh }\end{array}$ \\
\hline XJ(NRX, NTHMX2,LZMX2), & $\begin{array}{l}\text { Normalised Jacobian } \mathrm{J} / \mathrm{R} \\
\partial R\end{array}$ \\
\hline DRDU(), & $\overline{\partial u}$ \\
\hline $\operatorname{DRDS}()$ & $\frac{\partial R}{\partial S} \quad(\mathrm{~s} \equiv \mathrm{PSI})$ \\
\hline $\operatorname{DRDV}()$ & $\frac{\partial R}{\partial v}$ \\
\hline $\begin{array}{l}\text { BPSI }() \\
\operatorname{BMOD}()\end{array}$ & $\begin{array}{l}\mathrm{B}_{\psi}(\equiv 0) \\
|\mathrm{B}| \text { for calculations }\end{array}$ \\
\hline DBMODU(), & $\frac{\partial B}{\partial u}$ \\
\hline DBMODS(), & $\frac{\partial B}{\partial S}$ \\
\hline DBMODV() & $\frac{\partial B}{\partial v}$ \\
\hline DBMODUP(), & $\frac{\partial B}{\partial u}$ on semi mesh \\
\hline DBMODSP(), & $\frac{\partial B}{\partial S}$ on semi mesh \\
\hline DBMODVP() & $\frac{\partial B}{\partial}$ on semi mesh \\
\hline BMOD2() & $\mathrm{B}^{2}$ \\
\hline RGEOM(), & $\sqrt{X^{2}+Z^{2}}$ \\
\hline
\end{tabular}


THET2(NTHMX2),

C2(NRX,NTHMX2,LZMX2),

C3(),

BTAU(),

$\operatorname{BZETA}()$,

XIOTBR(),

XRG22(NRX,NTHMX2,LZMX2),

$\operatorname{RADR}()$

DC2DS()

DC2DU()

DG12DU()

DC2DV()

DG12DV()

DC3DU()

DJACDU()

DJACDV()

DG22DU() twice poloidal mesh

Normalised contravariant field $\frac{B^{U}}{B}$

on fine mesh

Normalised contravariant field $\frac{B^{V}}{B}$

on fine mesh

Normalised poloidal field $\frac{B^{U}}{B} \sqrt{g_{11}}$

on fine meshes

Normalised toroidal magnetic field $\frac{B^{V}}{B} \mathrm{R}$ on fine meshes

$$
\begin{aligned}
& \frac{B^{U}}{B^{V}} \\
& \sqrt{g_{22}}
\end{aligned}
$$

Real space coordinate $\mathrm{R}(\psi, \theta, \varphi)$ for calculations

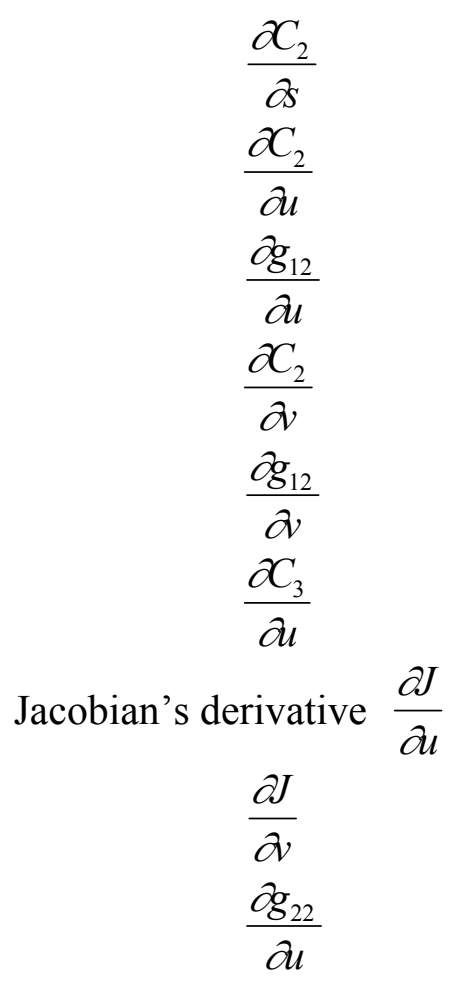




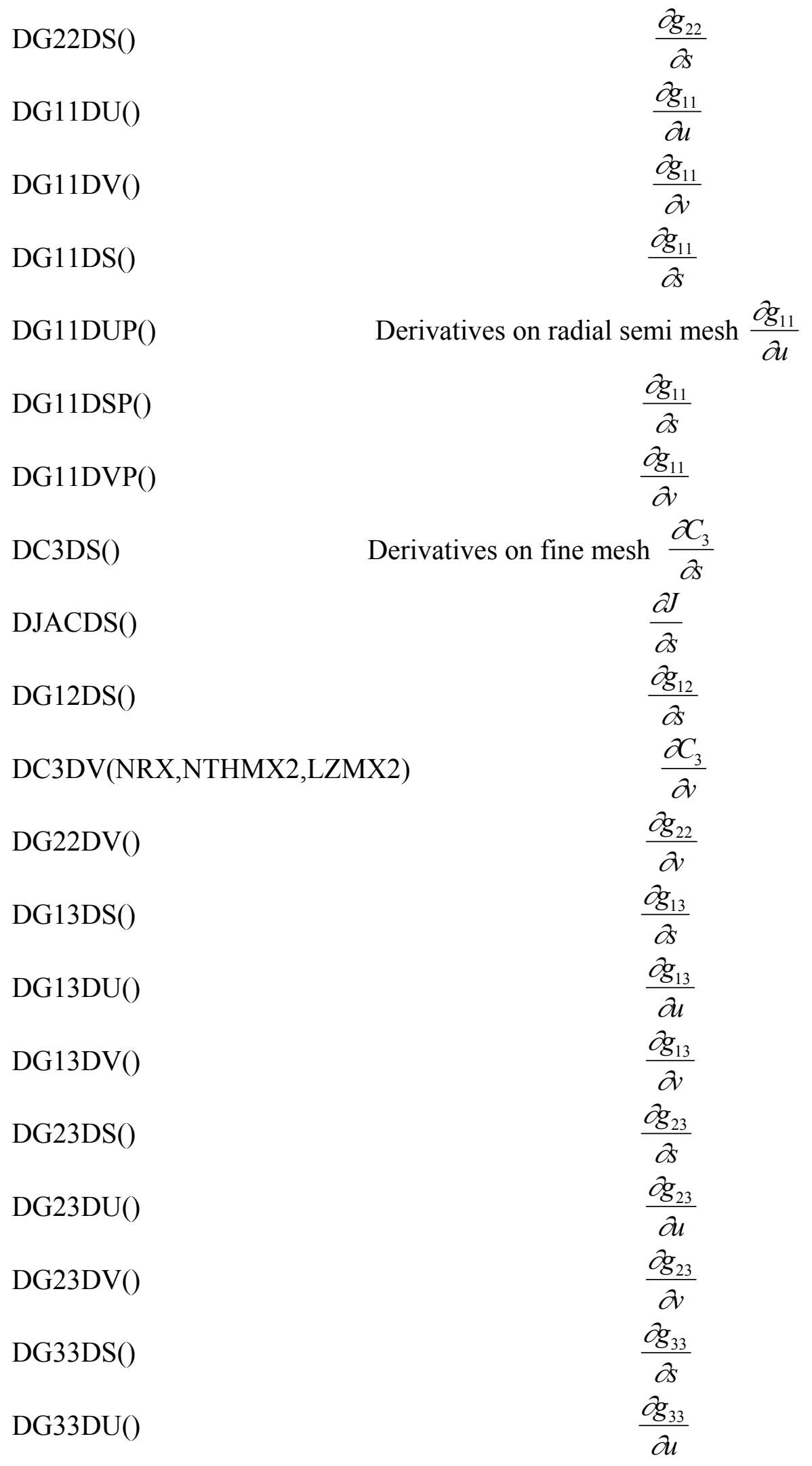


DG33DV()

DG13DSP()

DG13DUP()

DG13DVP()

DG23DSP()

DG23DUP()

DG23DVP()

DG33DSP()

DG33DUP()

DG33DVP()

XIOTBP(NRX,NTHMX2,LZMX2)

RADXP()

RADZP()

XG12P()

XRG11P(NRX,NTHMX2,LZMX2)

XRG22P()

VJACP()

RADRP(),

XJP()

$$
\frac{\partial g_{33}}{\partial v}
$$

Derivatives on fine semi mesh $\frac{\partial g_{13}}{\partial s}$

$$
\frac{\partial g_{13}}{\partial u}
$$

$\frac{\partial g_{13}}{\partial v}$

$\frac{\partial g_{23}}{\partial s}$

$\frac{\partial g_{23}}{\partial u}$

$\frac{\partial g_{23}}{\partial v}$

$\frac{\partial g_{33}}{\partial s}$

$\frac{\partial g_{33}}{\partial u}$

$\frac{\partial g_{33}}{\partial}$

$\frac{B^{U}}{B^{V}}$ on fine semi mesh

Real space coordinate $\mathrm{X}(\psi, \theta, \varphi)$ for calculations on semi mesh

Real space coordinate $Z(\psi, \theta, \varphi)$ for calculations on semi mesh

Metric tensor components on fine semi mesh: $g_{12}$

$$
\begin{gathered}
\sqrt{g_{11}} \\
\sqrt{g_{22}}
\end{gathered}
$$

Jacobian J on semi mesh \& others:

Real space coordinate $\mathrm{R}(\psi, \theta, \varphi)$ for calculations on semi mesh

Normalised Jacobian $\mathrm{J} / \mathrm{R}$ on semi mesh 


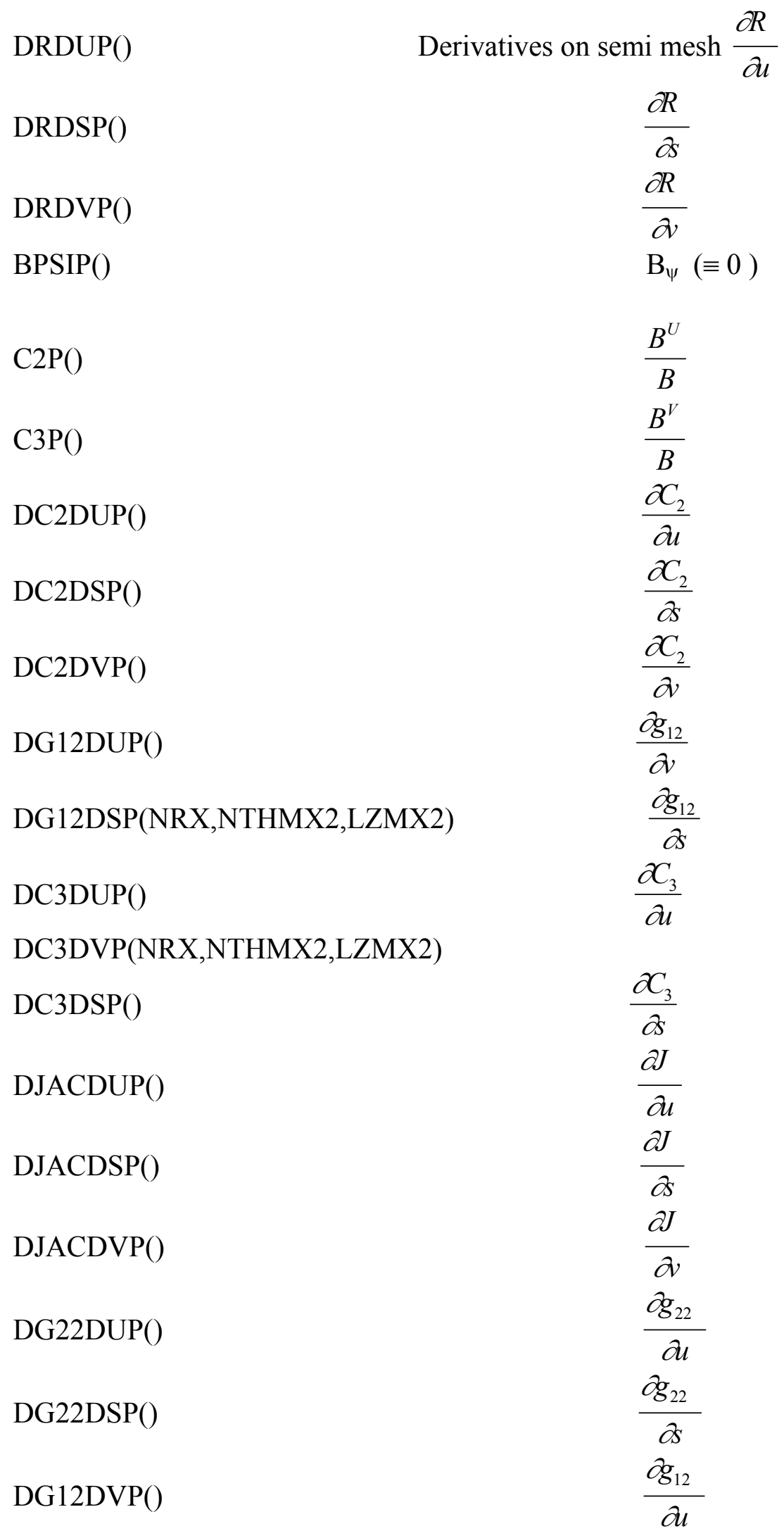


DG22DVP()

ROUT(NRX)

RIN(NRX)

ROUTPLT(NRX)

JPSI(NRX,NTHMX2,LZMX2)

JTAU()

$\operatorname{JZETA}()$

HTHETB(NTHMX2,LZMX2)

HTHETC(NTHMX2,LZMX2)

HTHETD(NTHMX2,LZMX2)

JR(NTHMX2,LZMX2),JTH(),JZ()

HMETRC(NTHMX2,LZMX2)

HMETR4(NTHMX4,LZMX4)

WORK2(NTHMX2)

WORK3(LZMX2)

WORK2D(NTHMX2,LZMIN2:LZMAX2)

WORK4(NTHMX4)

WORK5(LZMX4)

JRM(NRX,MPMIN2:MPMAX2,LZMX2)

$\operatorname{JTHM()}$

$\mathrm{XJZM}()$ $\frac{\partial g_{22}}{\partial v}$

Massive $\mathrm{R}(\mathrm{N}, 1,1)$

Massive $\mathrm{R}(\mathrm{N}, \mathrm{ntheta} / 2+1,1)$

Above two massives for plots

Complex "Radial" antenna specific exciting current

Complex simplified "quasi poloidal" antenna specific exciting current

Complex simplified toroidal antenna specific exciting current

Contribution to "Psi" specific antenna current:

$$
-i \omega \mu_{0} J_{\psi}^{a n t}
$$

Contribution to specific antenna poloidal current according to formula (...) in real space

Contribution to specific antenna toroidal current according to formula (...) in real space

Simplified antenna currents for FFTNs

Jacobian/R on fine mesh

Jacobian/R on twice fine mesh

Working massive

Working massive

Working massive

Working massive

Working massive

Poloidally Fourier transformed antenna radial current

Poloidally Fourier transformed antenna poloidal current

Poloidally Fourier transformed antenna toroidal current 
HMETM(NRX,MPMIN4:MPMAX4,LZMX2) Poloidally FFT of J/R on twice fine mesh JRML(NRX,MPMIN2:MPMAX2,LZMIN2:LZMAX2) Poloidally and toroidally Fourier transformed antenna radial current

JTHML()

JZML()

WDOTM(NRX)

PABSM(MPMIN:MPMAX)

WDOTML(NRX)
Poloidally and toroidally Fourier transformed antenna poloidal current Poloidally and toroidally Fourier transformed antenna toroidal current

Working massive

Poloidal spectrum of absorbed power

Working massive

PABSML(MPMIN:MPMAX,LZMIN:LZMAX) Poloidal and toroidal spectrum of absorbed power

D1(NTHMX2,LZMX2),.., D9(NTHMX2,LZMX2) Coefficients in ROT ROT E| $\left.\right|_{\psi}$ eqn. in real space

E1(NTHMX2,LZMX2),.., E6(NTHMX2,LZMX2) Coefficients in ROT ROT E $\left.\right|_{\psi}$ eqn. in real space

F1(NTHMX2,LZMX2),..., F9(NTHMX2,LZMX2) Coefficients in ROT ROT E $\left.\right|_{\psi}$ eqn. in real space

H1(NTHMX2,LZMX2),..., H9(NTHMX2,LZMX2) Coefficients in ROT ROT E $\left.\right|_{\theta}$ eqn. in real space

R1(NTHMX2,LZMX2),.., R6(NTHMX2,LZMX2) Coefficients in ROT ROT E $\left.\right|_{\theta}$ eqn. in real space

P1(NTHMX2,LZMX2),.., P9(NTHMX2,LZMX2) Coefficients in ROT ROT E $\left.\right|_{\theta}$ eqn. in real space

B1(NTHMX2,LZMX2),..,B5(NTHMX2,LZMX2) Coefficients in ROT ROT E| $\left.\right|_{\theta}$ eqn. in real space

S1(NTHMX2,LZMX2),..,S10(NTHMX2,LZMX2) Coefficients in ROT ROT E $\left.\right|_{\varphi}$ eqn. in real space

Z1(NTHMX2,LZMX2),..,Z10(NTHMX2,LZMX2) Coefficients in ROT ROT E $\left.\right|_{\varphi}$ eqn. in real space

Y1(NTHMX2,LZMX2),..,Y10(NTHMX2,LZMX2) Coefficients in ROT ROT E $\left.\right|_{\varphi}$ eqn. in real space 
D1M(NRX,MPMIN2:MPMAX2,LZMX2),.., D9M(NRX,MPMIN2:MPMAX2,LZMX2)

E1M(NRX,MPMIN2:MPMAX2,LZMX2),..,, E6M(NRX,MPMIN2:MPMAX2,LZMX2)

F1M(NRX,MPMIN2:MPMAX2,LZMX2),.., F9M(NRX,MPMIN2:MPMAX2,LZMX2)

H1M(NRX,MPMIN2:MPMAX2,LZMX2),.., H9M(NRX,MPMIN2:MPMAX2,LZMX2)

R1M(NRX,MPMIN2:MPMAX2,LZMX2),.., R6M(NRX,MPMIN2:MPMAX2,LZMX2)

B1M(NRX,MPMIN2:MPMAX2,LZMX2),..,, B5M(NRX,MPMIN2:MPMAX2,LZMX2)

P1M(NRX,MPMIN2:MPMAX2,LZMX2),.., P9M(NRX,MPMIN2:MPMAX2,LZMX2)

S1M(NRX,MPMIN2:MPMAX2,LZMX2),.., S10M(NRX,MPMIN2:MPMAX2,LZMX2)

Z1M(NRX,MPMIN2:MPMAX2,LZMX2),.., Z10M(NRX,MPMIN2:MPMAX2,LZMX2)

Y1M(NRX,MPMIN2:MPMAX2,LZMX2),.., Y9M(NRX,MPMIN2:MPMAX2,LZMX2)
Poloidal FFT of coefficients in ROT ROT E $\left.\right|_{\psi}$ eqn

Poloidal FFT of coefficients in ROT ROT E $\left.\right|_{\psi}$ eqn

Poloidal FFT of coefficients in ROT ROT E $\left.\right|_{\psi}$ eqn

Poloidal FFT of coefficients in ROT ROT E $\left.\right|_{\theta}$ eqn

Poloidal FFT of coefficients in ROT ROT E $\left.\right|_{\theta}$ eqn

Poloidal FFT of coefficients in ROT ROT E $\left.\right|_{\theta}$ eqn

Poloidal FFT of coefficients in ROT ROT E $\left.\right|_{\theta}$ eqn

Poloidal FFT of coefficients in ROT ROT E $\left.\right|_{\varphi}$ eqn

Poloidal FFT of coefficients in ROT ROT E $\left.\right|_{\varphi}$ eqn

Poloidal FFT of coefficients in ROT ROT E $\left.\right|_{\varphi}$ eqn

D1ML(NRX,MPMIN2:MPMAX2,LZMIN2:LZMAX2),.., Poloidal \& toroidal FFT of D9ML(NRX,MPMIN2:MPMAX2,LZMIN2:LZMAX2) coefficients in ROT ROT E| $\left.\right|_{\psi}$ eqn

E1ML(NRX,MPMIN2:MPMAX2,LZMIN2:LZMAX2),.., Poloidal \& toroidal FFT of E6ML(NRX,MPMIN2:MPMAX2,LZMIN2:LZMAX2) coefficients in ROT ROT E| $\left.\right|_{\psi}$ eqn

F1ML(NRX,MPMIN2:MPMAX2,LZMIN2:LZMAX2),.., Poloidal \& toroidal FFT of F9ML(NRX,MPMIN2:MPMAX2,LZMIN2:LZMAX2) coefficients in ROT ROT E $\left.\right|_{\psi}$ eqn

H1ML(NRX,MPMIN2:MPMAX2,LZMIN2:LZMAX2),.., Poloidal \& toroidal FFT of H9ML(NRX,MPMIN2:MPMAX2,LZMIN2:LZMAX2) coefficients in ROT ROT E $\left.\right|_{\theta}$ eqn R1ML(NRX,MPMIN2:MPMAX2,LZMIN2:LZMAX2),.., Poloidal \& toroidal FFT of 
R6ML(NRX,MPMIN2:MPMAX2,LZMIN2:LZMAX2) coefficients in ROT ROT E| $\left.\right|_{\theta}$ eqn

B1ML(NRX,MPMIN2:MPMAX2,LZMIN2:LZMAX2),.., Poloidal \& toroidal FFT of B5ML(NRX,MPMIN2:MPMAX2,LZMIN2:LZMAX2) coefficients in ROT ROT E| $\left.\right|_{\theta}$ eqn

P1ML(NRX,MPMIN2:MPMAX2,LZMIN2:LZMAX2),.., Poloidal \& toroidal FFT of P9ML(NRX,MPMIN2:MPMAX2,LZMIN2:LZMAX2) coefficients in ROT ROT E $\left.\right|_{\theta}$ eqn

S1ML(NRX,MPMIN2:MPMAX2,LZMIN2:LZMAX2),.., Poloidal \& toroidal FFT of S10ML(NRX,MPMIN2:MPMAX2,LZMIN2:LZMAX2) coefficients in ROTROT E| $\left.\right|_{\varphi}$ eqn

Z1ML(NRX,MPMIN2:MPMAX2,LZMIN2:LZMAX2),.., Poloidal \& toroidal FFT of Z10ML(NRX,MPMIN2:MPMAX2,LZMIN2:LZMAX2) coefficients in ROTROT E $\left.\right|_{\varphi}$ eqn

Y1ML(NRX,MPMIN2:MPMAX2,LZMIN2:LZMAX2),.., Poloidal \& toroidal FFT of Y9ML(NRX,MPMIN2:MPMAX2,LZMIN2:LZMAX2) coefficients in ROT ROT E| $\left.\right|_{\varphi}$ eqn

XBM(NRX,MPMIN2:MPMAX2,LZMX2)

XCM(NRX,MPMIN2:MPMAX2,LZMX2)

XDM(NRX,MPMIN2:MPMAX2,LZMX2)

Poloidal FFT to contribution to "radial" (psi) specific antenna current Poloidal FFT to contribution to poloidal specific antenna current

Poloidal FFT to contribution to toroidal specific antenna current

XBML(NRX,MPMIN2:MPMAX2,LZMIN2:LZMAX2) Poloidal \& toroidal FFTs to contribution to "radial" (psi) specific antenna current

XCML(NRX,MPMIN2:MPMAX2,LZMIN2:LZMAX2) Poloidal \& toroidal FFTs to contribution to poloidal specific antenna current

XDML(NRX,MPMIN2:MPMAX2,LZMIN2:LZMAX2) Poloidal \& toroidal FFTs to contribution to toroidal specific antenna current

XBMH(NRX,MPMIN2:MPMAX2,LZMX2) ) Poloidal FFT to contribution to radial specific antenna current on semi mesh

XBMLH(NRX,MPMIN2:MPMAX2,LZMX2) Poloidal \& toroidal FFTs to contribution to radial specific antenna current on semi mesh 
CEYFUN(NRX,NLMAX,NZMAX)

CEZFUN(NRX,NLMAX,NZMAX)
EX field component

Working array to calculate Fourier

EY field component

Working array to calculate Fourier EZ field component

CEX(NRX,MPMIN2:MPMAX2,LZMIN:LZMAX) Fourier representation of $E_{\psi}$ field over poloidal and toroidal angles

CEY(NRX,MPMIN2:MPMAX2,LZMIN:LZMAX) Fourier representation of $E_{\eta}$ field over poloidal and toroidal angles

CEZ(NRX,MPMIN2:MPMAX2,LZMIN:LZMAX) Fourier representation of $E_{\mathrm{b}}$ field over poloidal and toroidal angles

VJACOB(NRX,NTHMX2,LZMX2) Jacobian J on fine meshes

HMETM3(NRX,MPMIN4:MPMAX4,LZMIN4:LZMAX4) Poloidal \& toroidal FFTs of $\mathrm{J} / \mathrm{R}$ on twice fine mesh

VE11(NTHMX2,LZMX2),.., Intermediate working massives to

VE33(NTHMX2,LZMX2) calculate tensor components $\vec{\varepsilon}_{i j}$ in real space

$\begin{array}{ll}\text { WORK(NTHMX2) } & \text { Working massive } \\ \text { WORKZ(LZMX2) } & \text { Working massive }\end{array}$

CV3E11(MPMIN2:MPMAX2,MPMIN:MPMAX,LZMX2),.., Poloidal FFT of hot dielectric tensor $\vec{\varepsilon}_{i j}$

CV3E33(MPMIN2:MPMAX2,MPMIN:MPMAX,LZMX2)

CB5E11(MPMIN2:MPMAX2,LZMIN2:LZMAX2 Poloidal and toroidal FFTs

1 ,MPMIN:MPMAX,LZMIN:LZMAX),.., $\quad \vec{\varepsilon}_{i j}\left(m^{\prime}, n^{\prime}, m, n\right)$

CB5E33(MPMIN2:MPMAX2, LZMIN2:LZMAX2

1 ,MPMIN:MPMAX,LZMIN:LZMAX)

WORK(NTHMX2)

WORKZ(LZMX2)

Working massive

JPX(NRX,MPMIN2:MPMAX2,LZMIN2:LZMAX2)

Working massive

Fourier RF induced "psi"

plasma current component $\mathrm{J}_{\psi}$

JPY()

Fourier RF induced quasi 
poloidal current component $\mathrm{J}_{\eta}$

JPZ( )

XNEA(NRX)

XN1A(NRX), XN2A(NRX)

OMGCE(NRX, NTHMX2,LZMX2)

OMGCI2D(NRX,NTHMAX,LZTMAX)

OMGCI1(NRX,NTHMX2,LZMX2),

OMGCI2(NRX,NTHMX2,LZMX2)

OMGCED(NRX,NTHMAX,LZTMAX)

OMGP12(NRX)

OMGP22(NRX)

OMGPE2(NRX)

XN(NRX)

KTE(NRX)

KTI(NRX)

KTI2(NRX)

KPRL(NRX,NLMAX)

ADISP(NRX,NTHMX2)

BDISP(NRX,NTHMX2)

CDISP(NRX, NTHMX2)

KX2(NRX,NTHMX2)

KX2CD(NRX,NTHMX2)

KX2B(NRX, NTHMX2)
Fourier RF induced plasma current component along total confining magnetic field $\mathrm{J}_{\mathrm{b}}$ Electron density radial profile $n_{e}(s)$

Ion specious density arrays $\mathrm{n}_{\mathrm{i}}(\mathrm{s})$

Electron cyclotron frequency $\omega_{\mathrm{ce}}$ on fine mesh

Ion cyclotron frequency ion specious two for plotting

Ion cyclotron frequency ion specious 1 and 2 on fine mesh

Electron cyclotron frequency for plotting

$\omega_{\mathrm{pi}, 1}^{2}(\mathrm{~s})$

$\omega_{\mathrm{pi}, 2}^{2}(\mathrm{~s})$

$\omega_{\mathrm{pe}}^{2}(\mathrm{~s})$

$\mathrm{n}_{\mathrm{e}}(\mathrm{s})$

$\mathrm{kT}_{\mathrm{e}}(\mathrm{s})$

$\mathrm{kT}_{\mathrm{i}}(\mathrm{s})$ for bulk ions

$\mathrm{kT}_{\mathrm{i} 2}(\mathrm{~s})$ for minority ions

Local parallel wave number $\mathrm{K}_{/ /\left(\mathrm{s}_{\mathrm{n}}, \mathrm{m}\right)}$

Arrays A,B,C for local FW dispersion relation
Local $K_{\perp}^{2}$ for Fast Waves

$K_{\perp}^{2}$ for Fast Waves in cold plasma

$K_{\perp}^{2}$ for Ion Bernstein Waves 
KX20(NRX,NTHMX2)

KX2C0(NRX,NTHMX2)

KX2B0(NRX, NTHMX2)

KX2NL(NRX,NLMAX)

OMGRFC(NRX)

K2MOD(NRX,NTHMX2,LZMX2,

MPMIN:MPMAX,LZMIN:LZMAX)

XMI1

XMI2

XMEA

DEHST(NTHMX2)

JH1PS(NRX,NTHMX2,LZMX2)

JH1ET(NRX,NTHMX2,LZMX2)

JH1B(NRX, NTHMX2,LZMX2)

JH2PS(NRX,NTHMX2,LZMX2)

JH2ET(NRX,NTHMX2,LZMX2)

JH2B(NRX, NTHMX2,LZMX2)
$K_{\perp}^{2}$ for Fast Waves for specified poloidal number contributing to $\mathrm{K}_{/ /}$

$K_{\perp}^{2}$ for Fast Waves in cold plasma for specified poloidal number contributing to $\mathrm{K}_{/ /}$ $K_{\perp}^{2}$ for Fast Waves for specified poloidal number contributing to $\mathrm{K}_{/ /}$ Working array for current $K_{\perp}^{2}$

Complex generator frequency $\omega$

$K_{\perp}^{2}\left(s_{n}, \theta_{M}, \varphi_{L}, m, l\right)$

Majority ion mass M, S.I.

Minority ion mass M, S.I.

Electron mass, $\mathrm{m}_{\mathrm{e}}$, S.I.

FW Current Drive efficiency without trapped electrons effects

Plasma induced RF current $\mathrm{J}_{\psi}$ by ions specious 1 in real space

Plasma induced RF current $J_{\eta}$ by ions specious 1 in real space

Plasma induced RF current $\mathrm{J}_{\mathrm{b}}$ by ions specious 1 in real space

Plasma induced RF current $\mathrm{J}_{\psi}$ by ions specious 2 in real space

Plasma induced RF current $J_{\eta}$ by ions specious 2 in real space

Plasma induced RF current $\mathrm{J}_{\mathrm{b}}$ by ions specious 2 in real space 


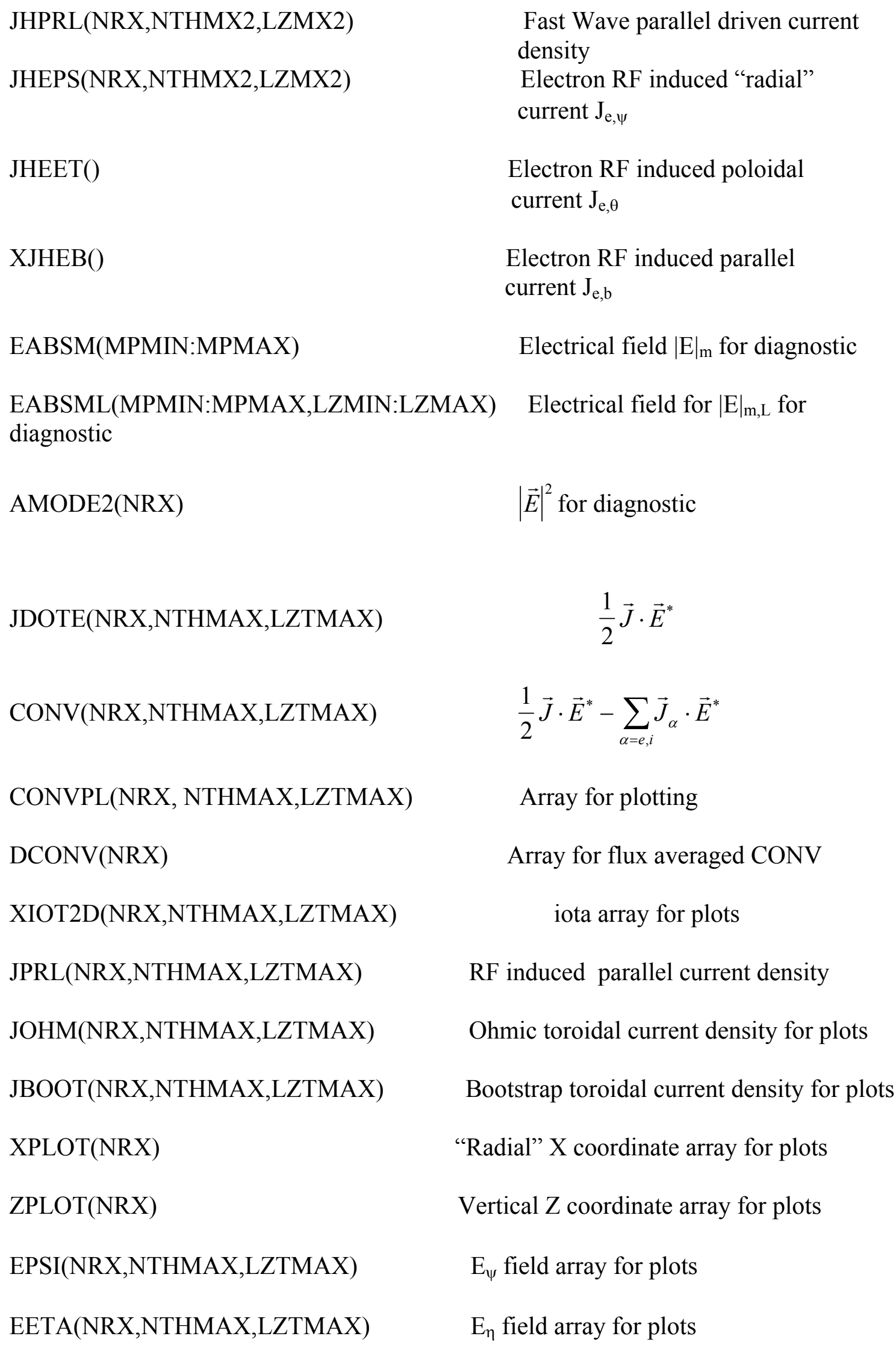




\begin{tabular}{|c|c|}
\hline EB(NRX,NTHMAX,LZTMAX) & $E_{b}$ field array for plots \\
\hline RE_EETA(NRX,NTHMAX,LZTMAX) & $\operatorname{Re}\left(E_{\eta}\right)$ field array for plots \\
\hline ER(NRX,NTHMAX,LZTMAX) & Radial electric field \\
\hline ETH(NRX,NTHMAX,LZTMAX) & Poloidal electric field \\
\hline EPHI(NRX,NTHMAX,LZTMAX) & Toroidal electric field \\
\hline JPPS(NRX,NTHMAX,LZTMAX,NSPEC) & $\begin{array}{l}\text { Radial RF plasma currents on semi mesh } \\
\text { for all ions specious }\end{array}$ \\
\hline JPET(NRX,NTHMAX,LZTMAX, NSPEC) & $\begin{array}{l}\text { Quasi poloidal RF plasma currents on } \\
\text { semi mesh for all ions specious }\end{array}$ \\
\hline JPB(NRX,NTHMAX,LZTMAX, NSPEC) & $\begin{array}{l}\text { Parallel RF plasma current on } \\
\text { semi mesh for all ions specious }\end{array}$ \\
\hline JP1PS(NRX,NTHMAX,LZTMAX) & $\begin{array}{l}\text { Radial RF plasma current on semi mesh } \\
\text { for majority ions (specious 1) }\end{array}$ \\
\hline JP1ET(NRX,NTHMAX,LZTMAX) & $\begin{array}{l}\text { Quasi poloidal RF plasma current on } \\
\text { semi mesh for ion specious } 1\end{array}$ \\
\hline JP1B(NRX,NTHMAX,LZTMAX) & $\begin{array}{l}\text { Parallel RF plasma current on } \\
\text { semi mesh for ion specious } 1\end{array}$ \\
\hline JP2PS(NRX,NTHMAX,LZTMAX) & $\begin{array}{l}\text { Radial RF plasma current on semi mesh } \\
\text { for minority ions (specious 2) }\end{array}$ \\
\hline JP2ET(NRX,NTHMAX,LZTMAX) & $\begin{array}{l}\text { Quasi poloidal RF plasma current on } \\
\text { semi mesh for minority ions (specious 2) }\end{array}$ \\
\hline JP2B(NRX,NTHMAX,LZTMAX) & $\begin{array}{l}\text { Parallel RF plasma current on } \\
\text { semi mesh for ion specious } 2\end{array}$ \\
\hline WDOT(NRX,NTHMAX,LZTMAX) & Total absorbed RF specific power $\dot{W}$ \\
\hline WDOT2(NRX,NTHMAX,LZTMAX) & $\begin{array}{l}\text { Specific power deposition to minority } \\
\text { ions } \dot{W}_{i 2}\end{array}$ \\
\hline DWDPSI(NRX) & $\begin{array}{l}\text { Total flux surface averaged "radial" } \\
\text { power deposition }\end{array}$ \\
\hline VOL(NRX,NTHMAX,LZTMAX) & Array for plasma volume normalisation \\
\hline FVDPSI(NRX) & Working array in poloidal averages \\
\hline
\end{tabular}


WDOTE(NRX,NTHMAX,LZTMAX)

WDEPLT(NRX,NTHMAX,LZTMAX)

DWEDPS(NRX,LZTMAX)

DWE_DPS(NRX) )

JEPLT(NRX,NTHMAX,LZTMAX)

EETPLT(NRX,NTHMAX,LZTMAX) plots

DJEDPS(NRX)

WDOTI1(NRX,NTHMAX,LZTMAX)

WDI1PL(NRX,NTHMAX,LZTMAX)

DW1DPS(NRX)

WDOTI2(NRX,NTHMAX,LZTMAX)

WDI2PL(NRX,NTHMAX,LZTMAX)

DW2DPS(NRX,LZTMAX)

DW2_DPS(NRX)

FWDPSI(NRX)

FWE_DPS(NRX)
Specific power deposition to electrons $\dot{W}_{e}$

Specific power deposition to electrons $\dot{W}_{e}$ for plots

Poloidally averaged power deposition to the electrons

Total flux surface averaged "radial" power deposition to the electrons

Plasma RF induced "parallel” electron current for plots

Quasi poloidal wave electric field $\mathrm{E}_{\theta}$ for

Flux surface averaged "radial" array for parallel electron current density

Specific power deposition to majority ions $\dot{W}_{i 1}$

Specific power deposition to majority ions $\dot{W}_{i 1}$ for plotting

Total flux surface averaged "radial" power deposition to the majority ions for plots

Specific power deposition to minority ions $\dot{W}_{i 2}$

Specific power deposition to minority ions $\dot{W}_{i 2}$ for plots

Poloidally averaged power deposition to the minority ions for plots

Total flux surface averaged "radial" power deposition to the minority ions for plots

Radial flux surface averaged total RF power deposition

Radial flux surface averaged RF power deposition to the electrons

Radial flux surface averaged RF power 
deposition to the majority ions

DWIDPS(NRX)

FWIDPS(NRX)

FW2_DPS(NRX)

FW2DPS(NRX,LZTMAX)

FWEDPS(NRX,LZTMAX)

J_N(LZMIN:LZMAX)

DWI2PLV(NRT)

DWEPLTV(NRT)

ETTAV(NRT)

PSI2DV(NRT)

dw2dpsv(nrx*lztmax $)$

dwedpsv(nrx*lztmax)
Flux surface averaged power deposition to the minority and majority ions for plots

Flux surface averaged power deposition to the minority and majority ions

Total flux surface averaged "radial" power deposition to the minority ions

Poloidally averaged "radial" power deposition to the minority ions

Poloidally averaged "radial" power deposition to the electrons

Antenna toroidal spectrum (in analytical approximation option)

Minority ions power absorption plotting array for writing in file (for future grafik)

Electrons power absorption plotting array for writing in a file (for future grafik) Quasi poloidal electric field $E_{\eta}$ plotting array for writing in a file (for future grafik)

Flux surfaces plotting array for writing in a file (for future grafik)

Poloidally averaged power depositions to minority ions (at different toroidal locations) for writing in a file

Poloidally averaged power depositions to the electrons (at different toroidal locations) for writing in a file

In the FLR code option flux surface averaged RF power deposition calculations the double Fourier harmonics summation under integral kernels in formulas (7.1) - (7.5) formally can be presented through an "effective" electrical field (involving separate summing over $m, l$ ) and an "effective" current (involving separate summing over prime indexes $m, l$ ') as $P=E_{\text {effec }}^{*} \cdot J_{\text {effec }}$. The respective "effective" variables for fields and currents have the names for ion fundamental, ion second harmonic, magnetic pumping, cross terms and Landau electron damping as 
>CEPERP_B, CJPERP_B, CETTMP, CJTTMP, CECROS1P, CECROS2P, $>$ CJCROS $\overline{1} \mathrm{P}, \mathrm{CJCROS} \overline{2} \mathrm{P}, \mathrm{CELANDP}, \mathrm{CJLANDP}, \mathrm{CEION} 2 \mathrm{P}, \mathrm{CJION} 2 \mathrm{P}$

Their mnemonic names permit easy recognition of respective identifiers. These effective variables are input for the power calculations in subroutine POWER_B. Their sizes are similar, e.g., to CEPERP_B (NRX, NTHMAX, LZTMAX), etc.

\subsection{Subroutine IBWCURE}

\section{Purpose:}

Does the calculation of RF wave induced plasma currents, with account to the electron and ions Finite Larmor Radius effects, and their Fourier transforms through the subroutines SIGMAEX_FLR $\left(\mathbf{J}_{\mathrm{i}, \mathrm{x}}^{(2)}+\mathbf{J}_{\mathrm{x}}^{(\mathrm{elec})}\right)$, SIGMAEY_FLR $\left(\mathbf{J}_{\mathrm{i}, \mathrm{y}}^{(2)}+\mathbf{J}_{\mathrm{y}}^{(\text {(elec) }}\right)$ and

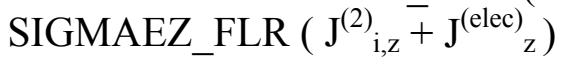

Use The calling sequence is CALL IBWCURE(N), N being flux surface numbering.

The major information produced by subroutine SIGMAXE_FLR in IBWCURE are the coefficients in discretized FLR equations (4.2.1), (4.2.2), (4.2.3), (4.3.1), (4.3.2) :

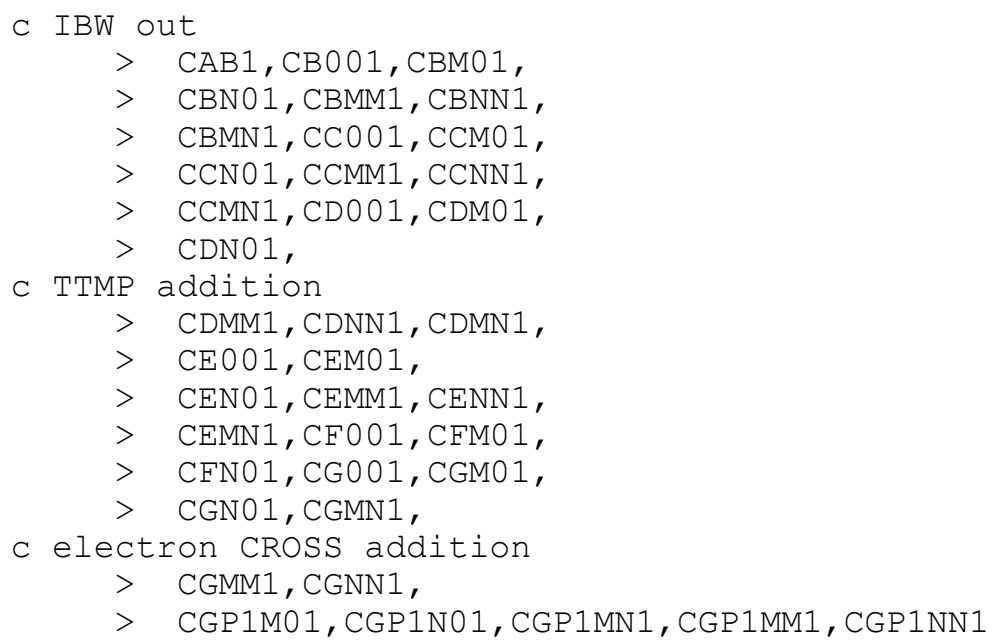

and the information given by subroutine SIGMAEY_FLR

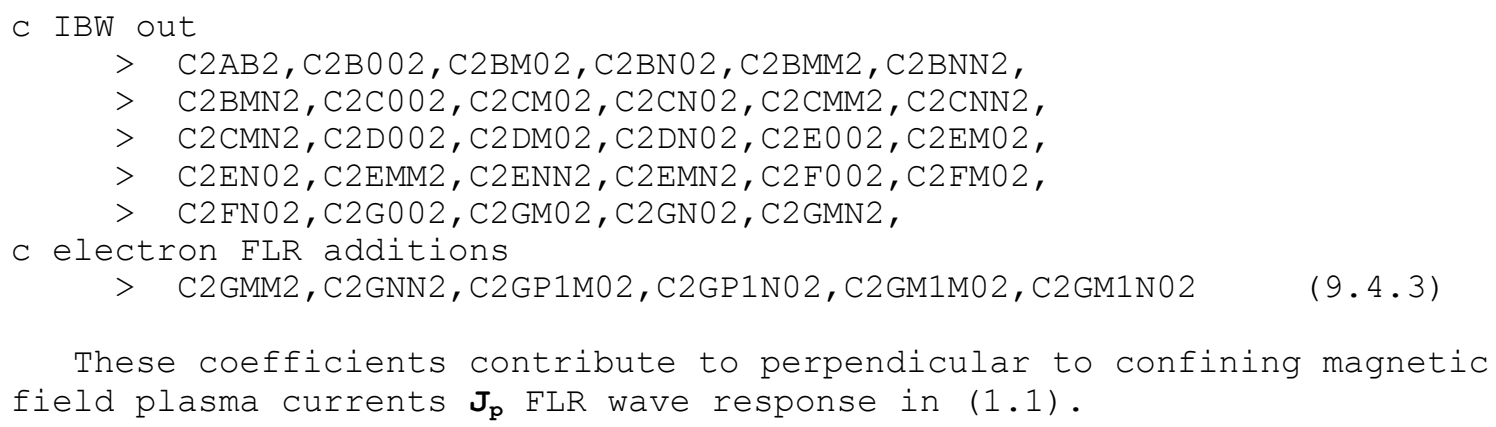


The FLR electron contributions to parallel plasma currents $J_{z}$ wave response are given by subroutine SIGMAEY_FLR providing the coefficients:

C IBW out with account to electrons CROSS terms inducing Jz current

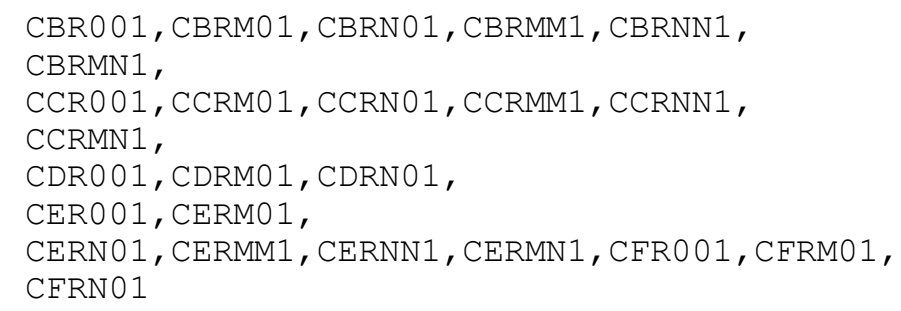

To calculate these coefficients the input information on DIVERGENCE and GRADIENT operators coefficients, involving metric and Jacobian, the $\alpha_{\mathrm{ij}}(2.2 .1)$ calculated in the GAMMAS_B and the related variables $\beta_{\mathrm{ij}}$ and $v_{\mathrm{ij}}$, due to reflection $\vec{R}$ matrix influence $((4.2 .4))$

$$
\begin{array}{lll}
\beta_{11}=J \alpha_{11} C_{2} & \beta_{12}=J\left(\alpha_{21} C_{2}+\alpha_{22} S_{2}\right) & \beta_{13}=J\left(\alpha_{31} C_{2}+\alpha_{32} S_{2}\right) \\
\beta_{21}=J \alpha_{11} S_{2} & \beta_{22}=J\left(\alpha_{21} S_{2}-\alpha_{22} C_{2}\right) & \beta_{23}=J\left(\alpha_{31} S_{2}-\alpha_{32} C_{2}\right) \\
v_{11}=-J \alpha_{11} S_{2} & v_{12}=J\left(-\alpha_{21} S_{2}+\alpha_{22} C_{2}\right) & \beta_{13}=J\left(-\alpha_{31} S_{2}+\alpha_{32} C_{2}\right) \\
v_{21}=J \alpha_{11} C_{2} & v_{22}=J\left(\alpha_{21} C_{2}+\alpha_{22} S_{2}\right) & v_{23}=J\left(\alpha_{31} C_{2}+\alpha_{32} S_{2}\right)
\end{array}
$$

are needed ones, together with their derivatives (also being calculated directly in the IBWCURE). These coefficients and their forward (indicated by letter"P"), centered and back (letter "M") derivatives in the code are presented by closely named variables:

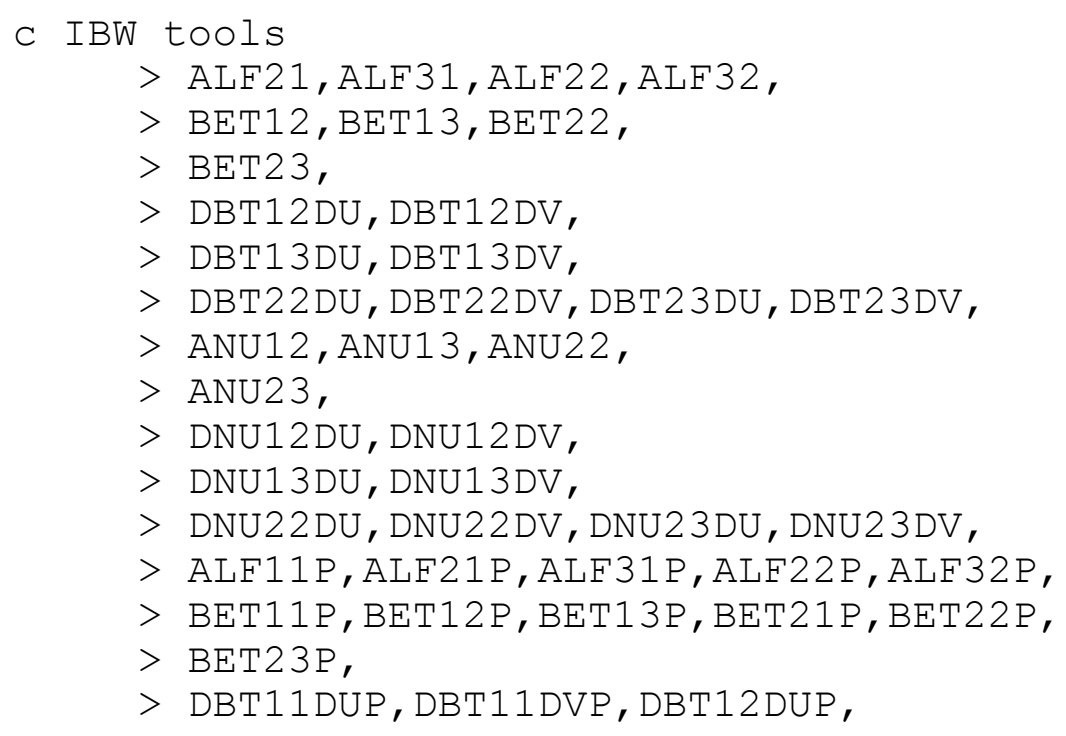




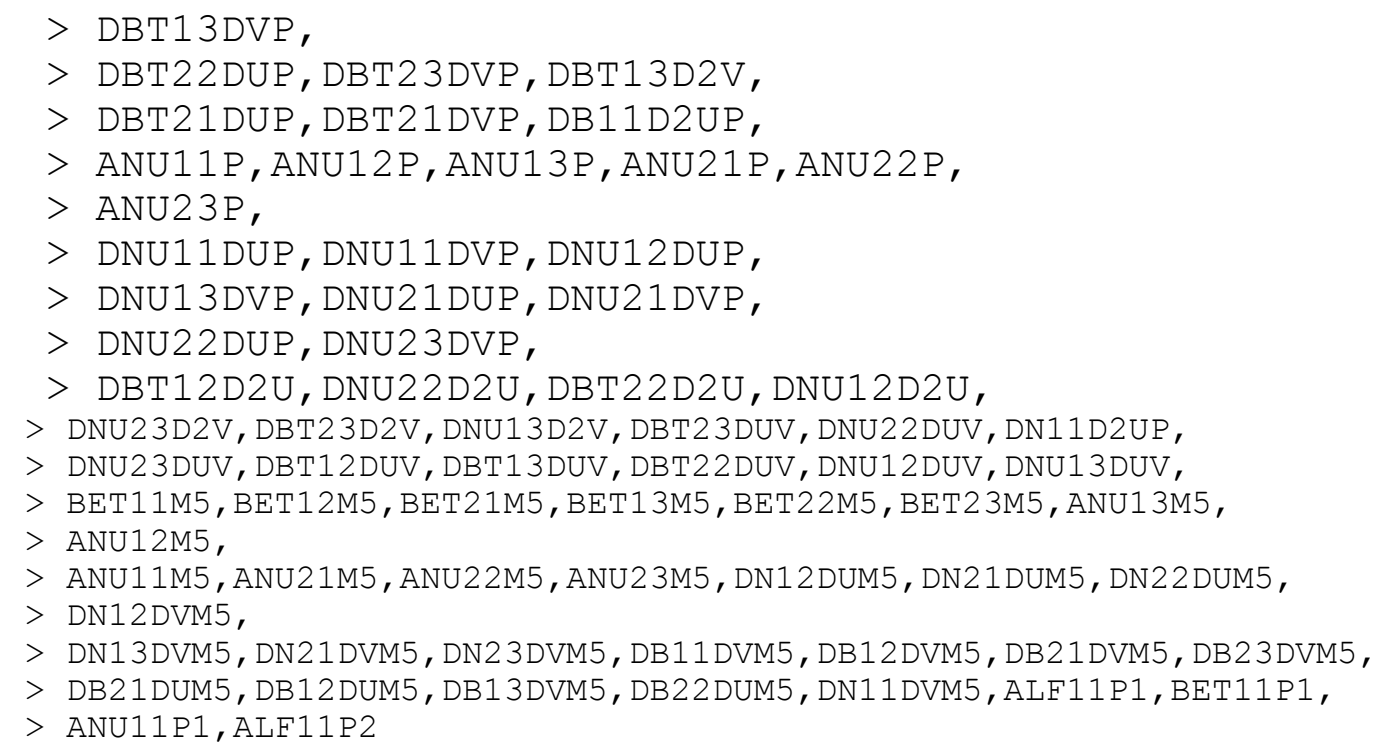

The finite ions temperatures coefficients $\sigma^{(2)}, \delta^{(2)}$ and electron temperatures coefficients $\lambda^{(0)}, \xi^{(0)}$ are calculated in IBWCURE by calling subroutines LAMBDA and LAMBEL. Their centered, forward or back radial grid point names in the code are:

$>\mathrm{CSG}, \mathrm{CSGP}, \mathrm{CDL}, \mathrm{CDLP}$,

$>$ CDLM1, CSGM1, CSGP2, CDLP2,

$>$ CLMBDO, CKSIO, CKSIOP

The flag IFLRE governs by electron FLR effects accounts: if IFLRE $=1$

Then electron FLR effects are treated through the second order operators. If IFLRE $=0$ then electron FLR effects are treated through the ORA approach.

Sizes of above variables can be found in common blocks described by include files:

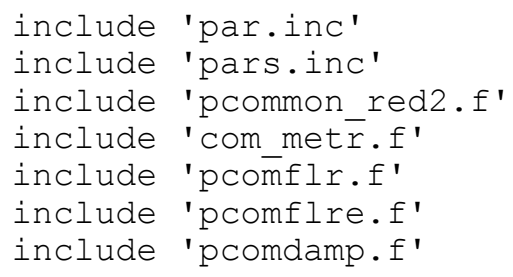

E.g. :

C IBW radial electron current Jx contribution COMMON / CURFLRe/

$>\operatorname{CDMM}(\mathrm{NTHMX} 2, \operatorname{LZMX} 2)$,

$>$ CDNN (NTHMX2, LZMX2), CDMN (NTHMX2, LZMX2),

$>$ CGMM (NTHMX2, LZMX2), CGNN (NTHMX2, LZMX2)

and so on.

When calculated these multiple variables are replaced by their FFT 
transforms. Respective FFT reworked variables add letters $M$ or ML at the end of above multiple names.

In the ORA approximation the subroutine IBWCURE is not called one. Calling the TENSOR subroutine is sufficient one.

\subsection{Subroutines filling tridiagonal stiffness matrix}

Purpose

VMATRIX_B_EL = evaluation and non zero elements filling a basic linear problem matrix (to be inverted) with account to FLR effects for the stellarators problem, 3D open traps may be an option).

VMATRIX_RED2 = evaluation and non zero elements filling a basic linear problem matrix (to be inverted) with the ORA code option account to FLR effects for the stellarators (3D problem, 3D open traps may be an option).

VMATRTOK_B_EL = evaluation and non zero elements filling a basic linear problem matrix (to be inverted) with account to FLR effects for the tokamaks (2D problem, 2D open traps may be an option).

VMATRTOK_RED2 = evaluation and non zero elements filling a basic linear problem matrix (to be inverted) with the ORA code option account to FLR effects for the tokamaks (2D problem, 2D open traps may be an option).

These subroutines fill tri blocks of stiffness matrix CB1, CB2 and CB3:

CB1_block(-1:1,NLMAX3,NZMAX) Working array to fill main matrix with contribution of PSI component to wave equation

CB2_block(-1:1,NLMAX3,NZMAX) Working array to fill main matrix with contribution of ETA component to wave equation

CB3_block(-1:1,NLMAX3,NZMAX) Working array to fill main matrix with contribution of "parallel" component to wave equation

Output comes through the common/MATR/ .

The code also contains additional subroutines, functions and common blocks not listed above.

\section{Conclusions}


In this report we described theory and 3D full wave code description for the wave excitation, propagation and absorption in 3-dimensional (3D) stellarator equilibrium high beta plasma in ion cyclotron frequency range (ICRF). This theory formed a basis for a $3 \mathrm{D}$ code creation, urgently needed for the ICRF heating scenarios development for the operated LHD [1], constructed W7-X [2], NCSX [3] and projected CSX3 [3] stellarators, as well for re evaluation of ICRF scenarios in operated tokamaks and in the ITER. The theory solves the 3D Maxwell-Vlasov antenna-plasma-conducting shell boundary value problem in the non - orthogonal flux coordinates. All basic physics, like wave refraction, reflection and diffraction are self consistently included, along with the fundamental ion and ion minority cyclotron resonances, two ion hybrid resonance, electron Landau and TTMP absorption. This is accomplished in a real confining magnetic field being varying in a plasma major radius direction, in toroidal and poloidal directions, through making use of the hot dense plasma wave induced currents with account to the finite Larmor radius effects.

The theory was developed in a manner that includes tokamaks and magnetic mirrors as the particular cases through general metric tensor (provided by an equilibrium solver) treatment of the wave equations.

We described newly developed stellarator ICRF 3D full wave code PSTELION, based on theory described in this report. Application to tokamaks, ITER and stellarators is given in attached subreports $[15,16,17]$.

\section{References}

1. A.Iiyoshi, M.Fujiwara, O.Motojima, N.Ohybu, K.Yamazaki Fusion Technology 17(1990) 169

2. WENDELSTEIN project Group, IPP-EURATOM Association, WENDELSTEIN 7-X, Phase II, Application for Preferencial Support, June 1994

3. G.Neilson, et al., PPPL-3676 (2002)

4. V.Vdovin, T.Watari, A.Fukuyama, Proc.ICPP 96 (Nagoya 1996),1070; NIFS- 469

5. Brambilla M. Plasma Physics Controlled Fusion 31 (1989), 723

6. Brambilla M. Plasma Physics Controlled Fusion 41 (1999), 1

7. Jaeger E.F. et all, Physics of Plasma, 9 (2002), N9, 1873 ;

J. C. Wright, P. T. Bonoli, M. Brambilla, F. Meo, E. F. D'Azevedo, D. B. Batchelor, E. F. Jaeger, L. A. Berry, C. K. Philips, and A. Pletzer. Full wave simulations of fast wave mode conversion and lower hybrid wave propagation in tokamaks. Physics of Plasma, 11, 2473 (2004)

8. Akhiezer A. et al, "Plasma Electrodynamics" (Publisher: "Science", 
Moscow,1974 )

9. Swanson D.G. Physics Fluids 24 (1981), 2035

10. Colestock P.L., Kashuba R.J. Nucl. Fusion 23 (1983), 763

11. Brambilla M. Phys.Lett. 188A (1994), 376

12. Brambilla M and Krucken, Nucl. Fusion 28, 1813 (1988)

13. Brambilla M. Nucl. Fusion 38, 1805 (1998)

14. Technical Basis for the ITER Final Design Report, JCT Garching-Naka, ITER scenarios 2003 from JCT

15. Vdovin V. Study of HFFW heating and current drive in the DIII-D tokamak QDB scenario, attachment \#3 to this report (February 2005)

16. Vdovin V. ICRF Plasma Heating in Large Stellarators (includes Detailed simulation of ICRF scenarios and antennae impedance characteristics for stellarator NCSX), attachment \#1 to this report (February 2005)

17. Vdovin V. ICRF benchmarking modelling of ITER scenario \#2 (attachment \#4 to this report (June 2005))

18. Hutchinson I.H. et al., Plasma Physics, 1, 1511 (1994) 


\title{
ICRF Plasma Heating in Large Stellarators
}

\author{
Vdovin V.L. \\ RRC Kurchatov Institute, Nuclear Fusion Institute \\ Moscow, Russia
}

\section{Topics}

- Stellarators equilibriums and antennae

- Tokamak-like and "magnetic beach" scenarios

- Recent ICRF experiments in large stellarators

- New ICRF Physics in stellarators

- 3D full wave ICRF code and plasma heating modelling

- ICRF in Large and novel stellarators projects

- Conclusions 


\begin{abstract}
In the paper we numerically model Ion Cyclotron Plasma Heating (ICRF) scenarios with 3D Full Wave ICRF PSTELION code in operating large LHD and Advanced stellarators W7AS, constructed W7-X and NCSX with improved neoclassical confinement through quasi and quasi axis symmetry concepts. All basic ICRF scenarios are analyzed and frequency bands were found and are recommended for an engineering design. Preliminary results were reported in [6]. The ICRF is now only available method for steady state plasma heating in stellarators.
\end{abstract}

\title{
Largest world stellarators
}

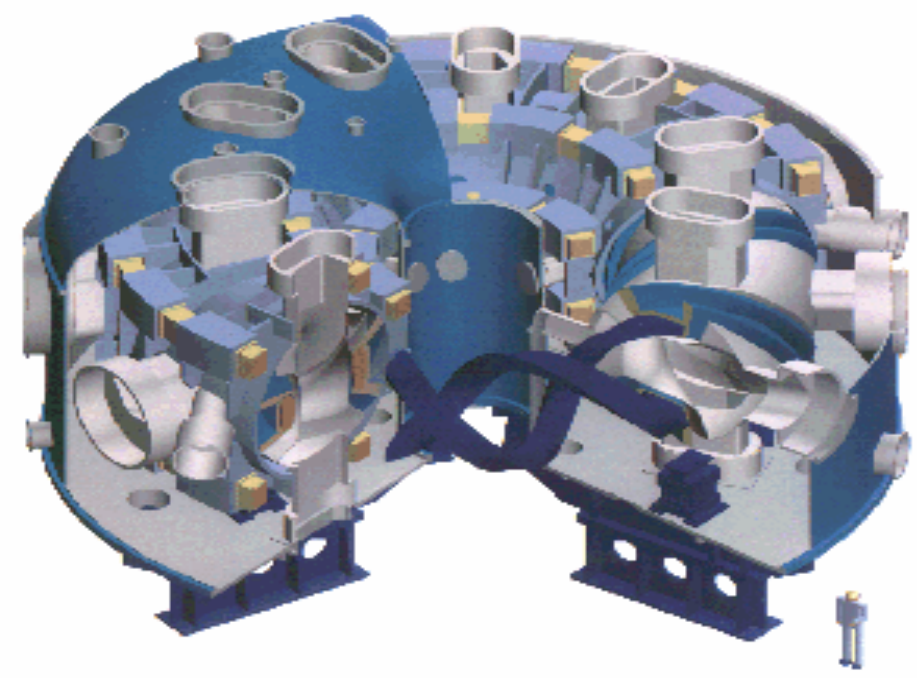

LHD (Japan) - largest world currently operating torsatron $\mathrm{A}=6.5, \mathrm{R}=3.9 \mathrm{~m}, \mathrm{~N}=10$ 


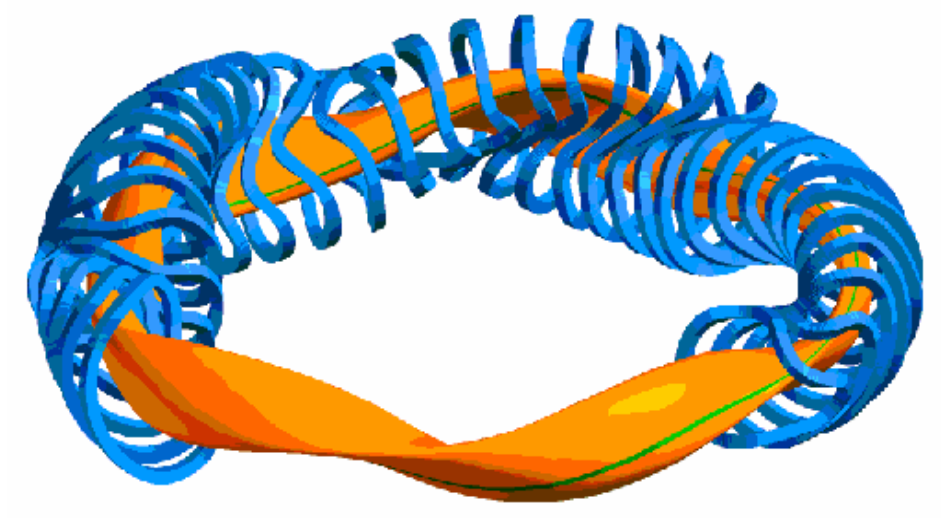
W7-X (Germany) advanced stellarator (constructed) $\mathrm{A}=10, \mathrm{R}=5.5 \mathrm{~m}, \mathrm{~N}=5$

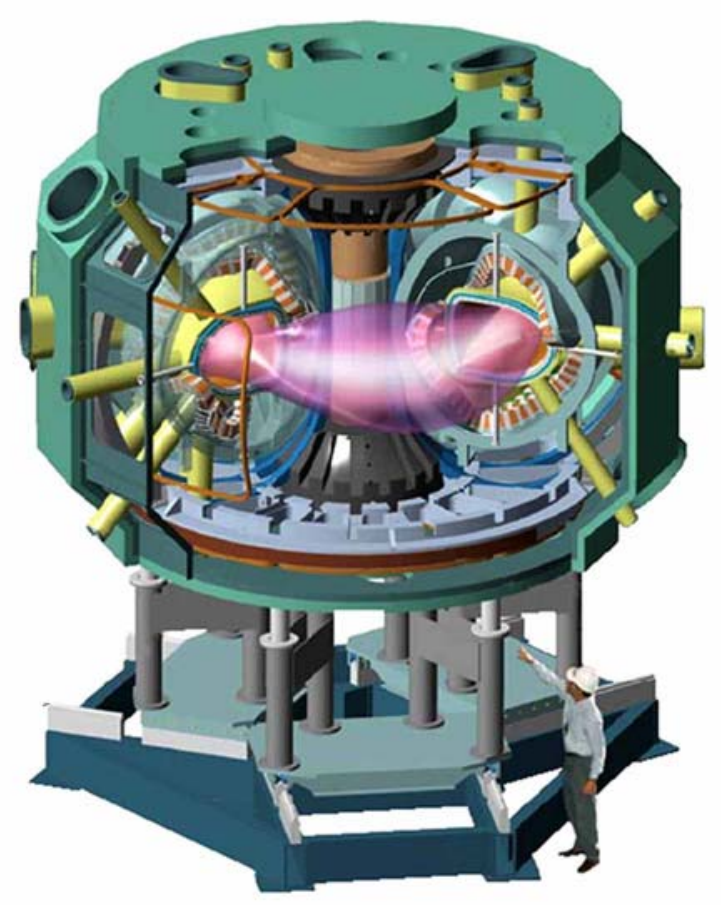

NCSX (USA) advanced (quasi-axis symmetry) Stellarator (constructed) $\mathrm{A}=4.4, \mathrm{R}=1.4 \mathrm{~m}, \mathrm{~N}=3$ 


\section{Stellarators' equilibriums and antennae}

The ICRF method is using outside and inside poloidal multi loop and frame type antennae as is shown in Fig.1 below for W7-AS plasma.

- W7-AS example 


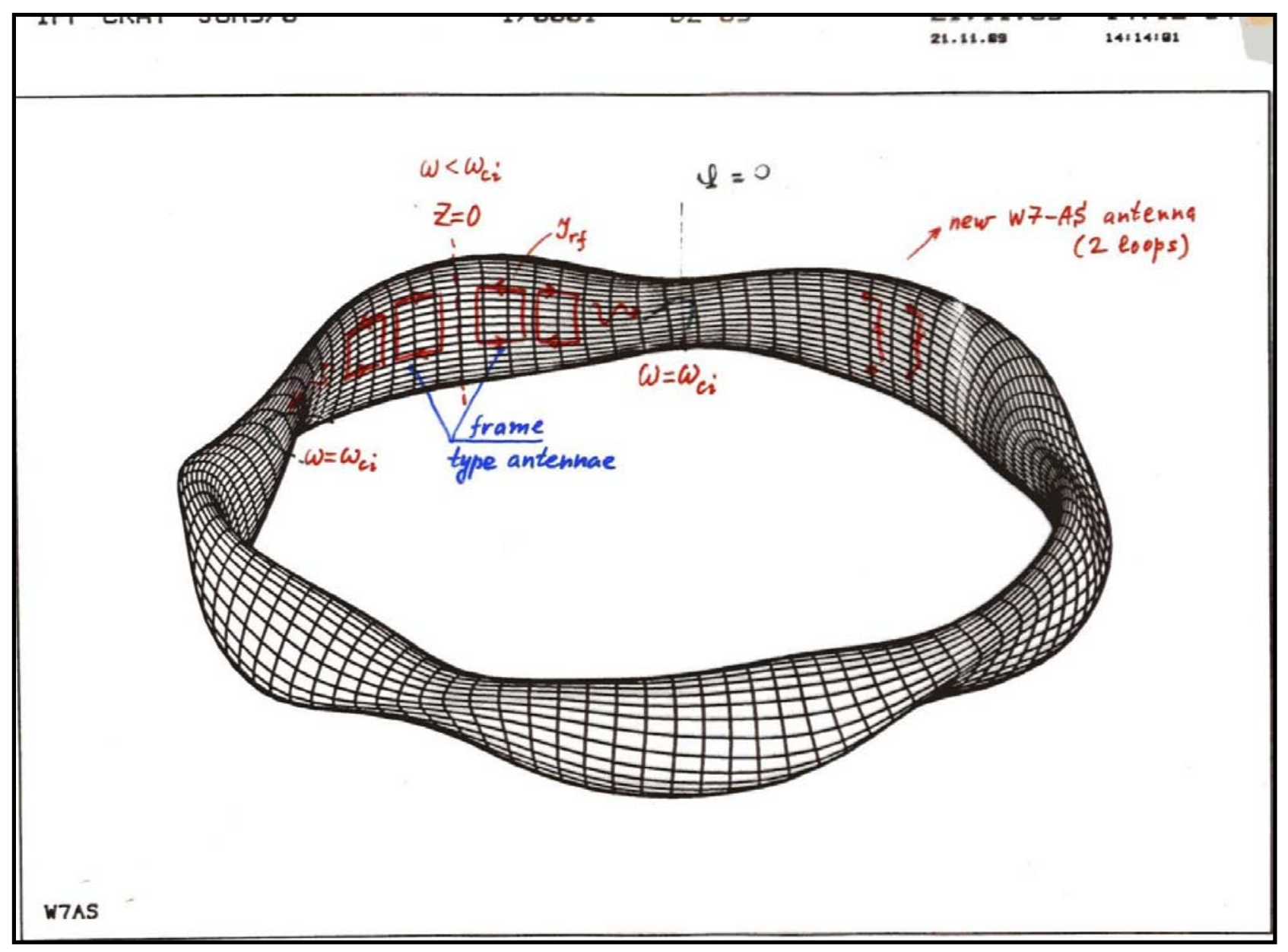

Fig.1 ICRF antennae frequently used in stellarators and torsatrons (W7-AS plasma example).

\section{New ICRF physics in stellarators}




\section{New favourable ICRF Physics in stellarator}

- Parallel (to B) Wave shortening

- Toroidal modes coupling

- Existence of "Mode families"

- Due to double toroidal periodicity in stellarators

toroidal harmonics are coupled through the condition:

$$
\mathrm{nN}_{\mathrm{p}}+\mathrm{n}-\mathrm{k}=0
$$

being some kind analog of axis symmetry of tokamaks reflected to the stellarators (Nuhrenberg 1995)

Parallel Wave shortening effect

- increases wave absorption strength

1) Broadness of Doppler Resonance rises:

$$
\Delta \mathbf{Z}=\mathbf{Z}_{\mathbf{0}} \mathbf{K}_{/ /} \mathbf{V}_{\mathrm{Ti}} / \boldsymbol{\omega}
$$

2) Phase resonance with thermal electrons at a beach:

$$
\mathbf{V}_{\mathrm{ph}}=\omega / \mathbf{K}_{/ /} \approx \mathbf{V}_{\mathrm{Te}}
$$

while under antenna: $\omega / \mathbf{K}_{/ /}>\mathbf{V}_{\mathrm{Te}}$

Example of parallel wave behavior in mirror

- axial electron absorption distribution 


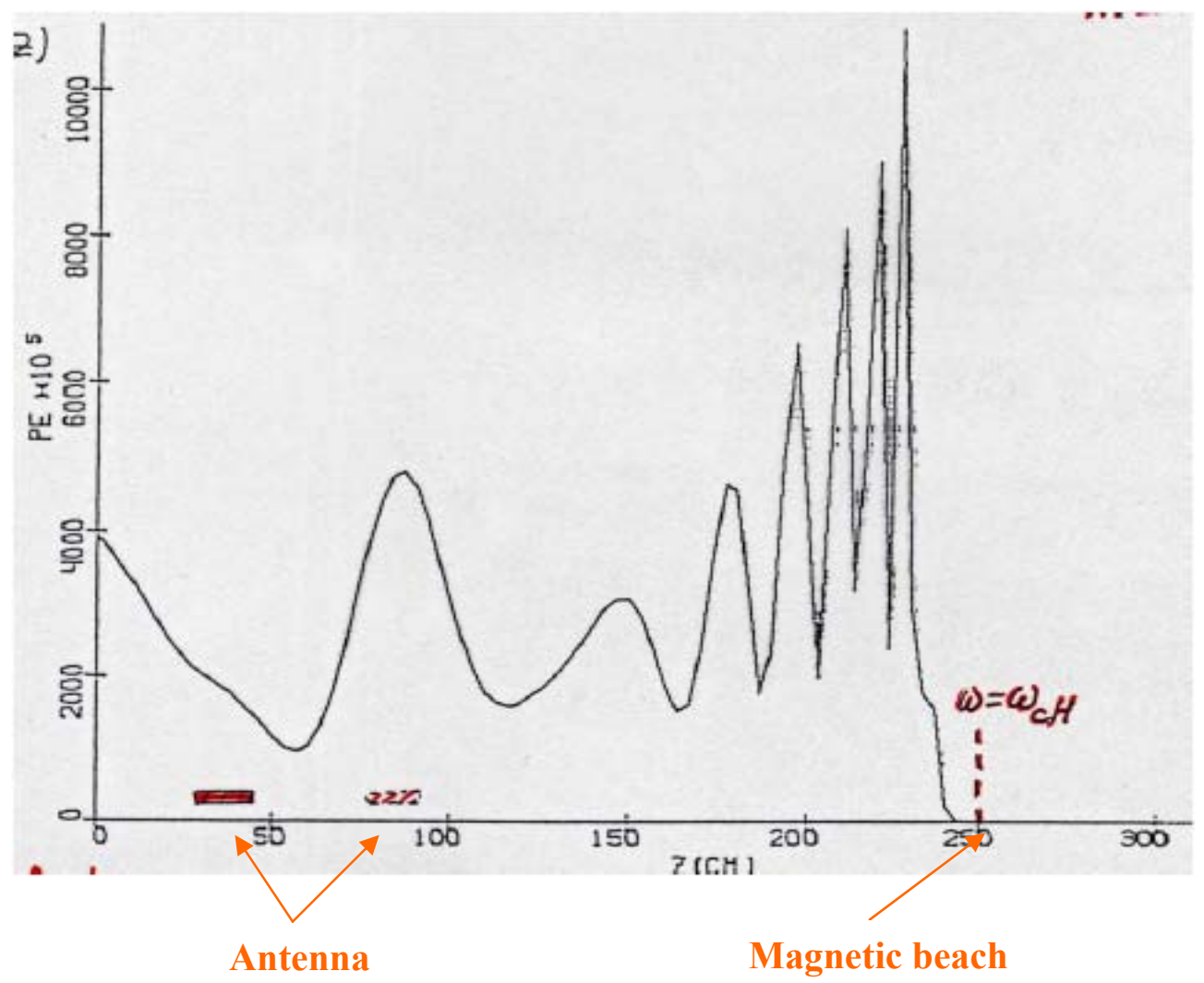

Fig. 2.1 Parallel fast wave length shortening when approaching $\omega=\omega_{\mathrm{cH}}$ Magnetic beach

$$
\begin{aligned}
& A=30 \mathrm{~cm} \\
& B 0=2.5 \mathrm{~T} \\
& N_{\mathrm{e} 0}=1.210^{20} \mathrm{~m}-3 \\
& D(10 \% \mathrm{H}) \\
& T_{\mathrm{i} 0}=980 \mathrm{eV} \\
& T_{\mathrm{e} 0}=500 \mathrm{eV} \\
& \mathrm{Qi} \leq \mathbf{Q e} \\
& M=+1 \text { (poloidal number) }
\end{aligned}
$$


used in PSTELION modelling. This convenient to present by the Table:

\begin{tabular}{|c|c|c|c|c|}
\hline & W 7-AS & LHD & W7-X & NCSX \\
\hline $\begin{array}{c}\text { Major } \\
\text { radius, } m\end{array}$ & 2.0 & 3.6 & 5.5 & 1.42 \\
\hline $\begin{array}{c}\text { Minor } \\
\text { Radius, } m\end{array}$ & 0.18 (avg) & 0.55 & 0.5 & 0.33 (avg) \\
\hline $\begin{array}{l}\text { Electron } \\
\text { density, } \mathrm{m}^{-3}\end{array}$ & $510^{19}$ & $310^{19}$ & $510^{19}$ & $610^{19}$ \\
\hline $\begin{array}{l}T_{\text {e0 }}, \mathrm{keV} \\
T_{\mathrm{i} 0}, \mathrm{keV}\end{array}$ & $\begin{array}{l}2.5 \\
0.5\end{array}$ & $\begin{array}{l}2.0 \\
2.0\end{array}$ & $\begin{array}{l}3.0 \\
3.0\end{array}$ & $\begin{array}{l}2.3 \\
2.3\end{array}$ \\
\hline $\begin{array}{l}\text { Magnetic } \\
\text { Field, T }\end{array}$ & $1.25-2.5$ & 2.7 & 2.5 & 1.7 \\
\hline $\begin{array}{l}\text { Generator } \\
\text { power, Mw } \\
\text { Frequency } \\
\text { range, } \mathrm{MHz}\end{array}$ & $\begin{array}{c}1.2 \\
34-74\end{array}$ & $\begin{array}{c}2 \times 1.5 \\
25-100\end{array}$ & $\begin{array}{l}2 \times 1.5 \\
25-76\end{array}$ & $\begin{array}{l}\text { TBD } \\
\text { TBD }\end{array}$ \\
\hline $\begin{array}{l}\text { Antenna, } \\
\text { location }\end{array}$ & $\begin{array}{l}\text { Double } \\
\text { Strap } \\
\text { HFS }\end{array}$ & $\begin{array}{l}\text { Mono Strap } \\
\text { array } \\
\text { LFS (HFS) }\end{array}$ & $\begin{array}{l}\text { Double } \\
\text { Strap } \\
\text { LFS }\end{array}$ & $\begin{array}{l}\text { Double } \\
\text { Strap (?) } \\
\text { LFS }\end{array}$ \\
\hline & & & & \\
\hline
\end{tabular}


The Large Helical Device (LHD) has shown impressive ICRF plasma heating by minimizing amount of helically trapped ions by inward shift of magnetic axis. This was demonstrated by $68 \mathrm{sec}$ shot traces in minority heating scenario $(7 \% \mathrm{H}+\mathrm{He})$ with achieved $\mathrm{T}_{\mathrm{i}}=\mathrm{T}_{\mathrm{e}}=2 \mathrm{keV}$ [1] and $\sim 1 \mathrm{keV}$ in $120 \mathrm{sec}$ pulse (Fig.1a) through creation of well confined energetic proton tail up to $500 \mathrm{keV}$ (Fig.1b) [2]. Power was raised to $2.7 \mathrm{MW}$ using several antennae. Recently duration pulses were prolongated up to 32 minutes leading to world record energy radiated into plasma: $1.3 \mathrm{GJ}$ [2a]. The investigated ICRF scenarios are:

$\checkmark$ ICRF power up to $2.7 \mathrm{MW}$ (six antennae)

$\checkmark$ Minority ion heating

- best performance

- stored energy up to $240 \mathrm{~kJ}$

- inward-shifted magnetic axis

- observed high energy ions up to $500 \mathrm{keV}$

$\checkmark$ Second harmonic Heating

- Slow Wave heating

$\checkmark$ Fundamental Harmonic Heating

- plasma density range up to $110^{20} \mathrm{~m}-3$

- firstly in Large tokamaks/stellarators 
Resonance and Cut-off surfaces in LHD cross section in minority ion mode

$(B O=2.75 T, 38.5 \mathrm{MHz}, \mathrm{He}(\mathrm{H}: 30 \%)$ plasma, $\mathrm{k} / /=5 \mathrm{~m}-1)$

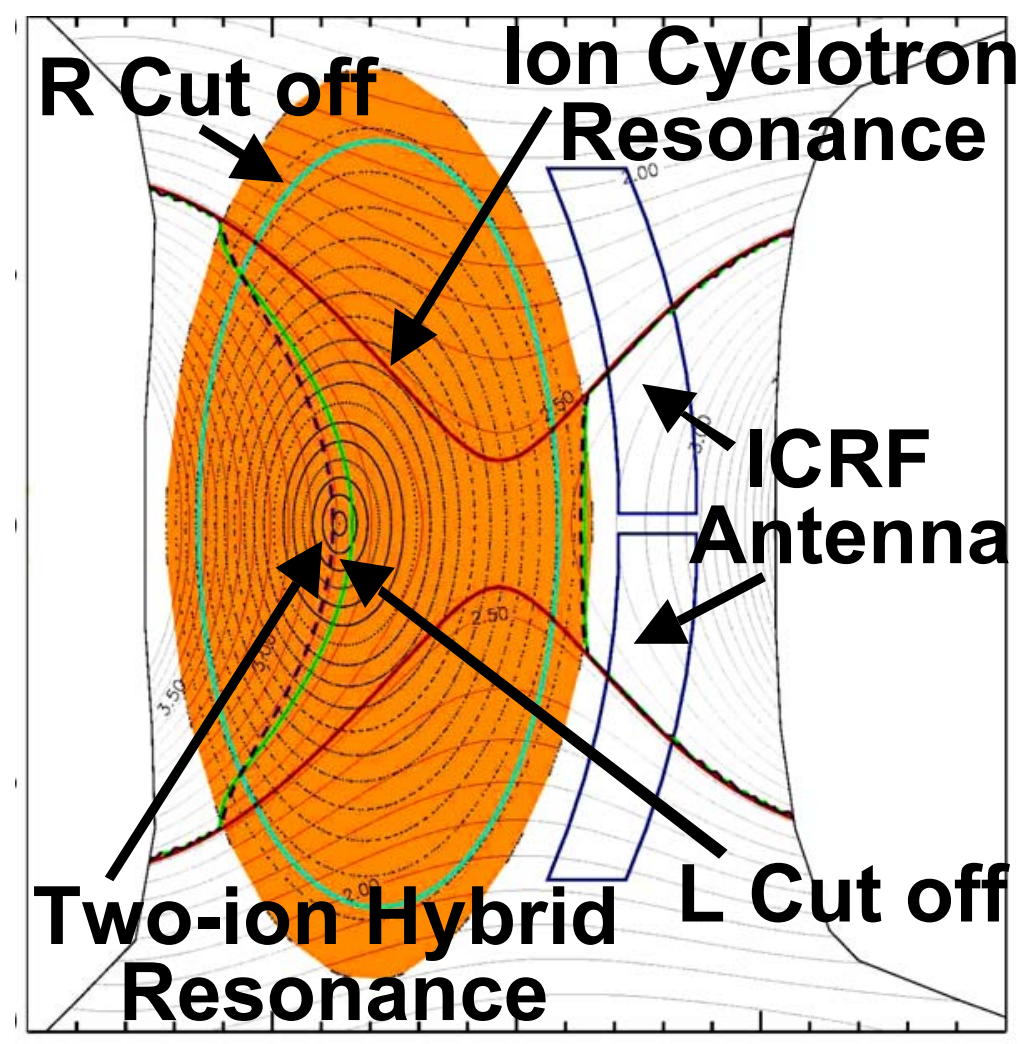

Fig. 3.1 
Long pulse plasma sustainment in LHD by ICRF for 2 minutes (and recently sustained up to 32 minutes [2a])

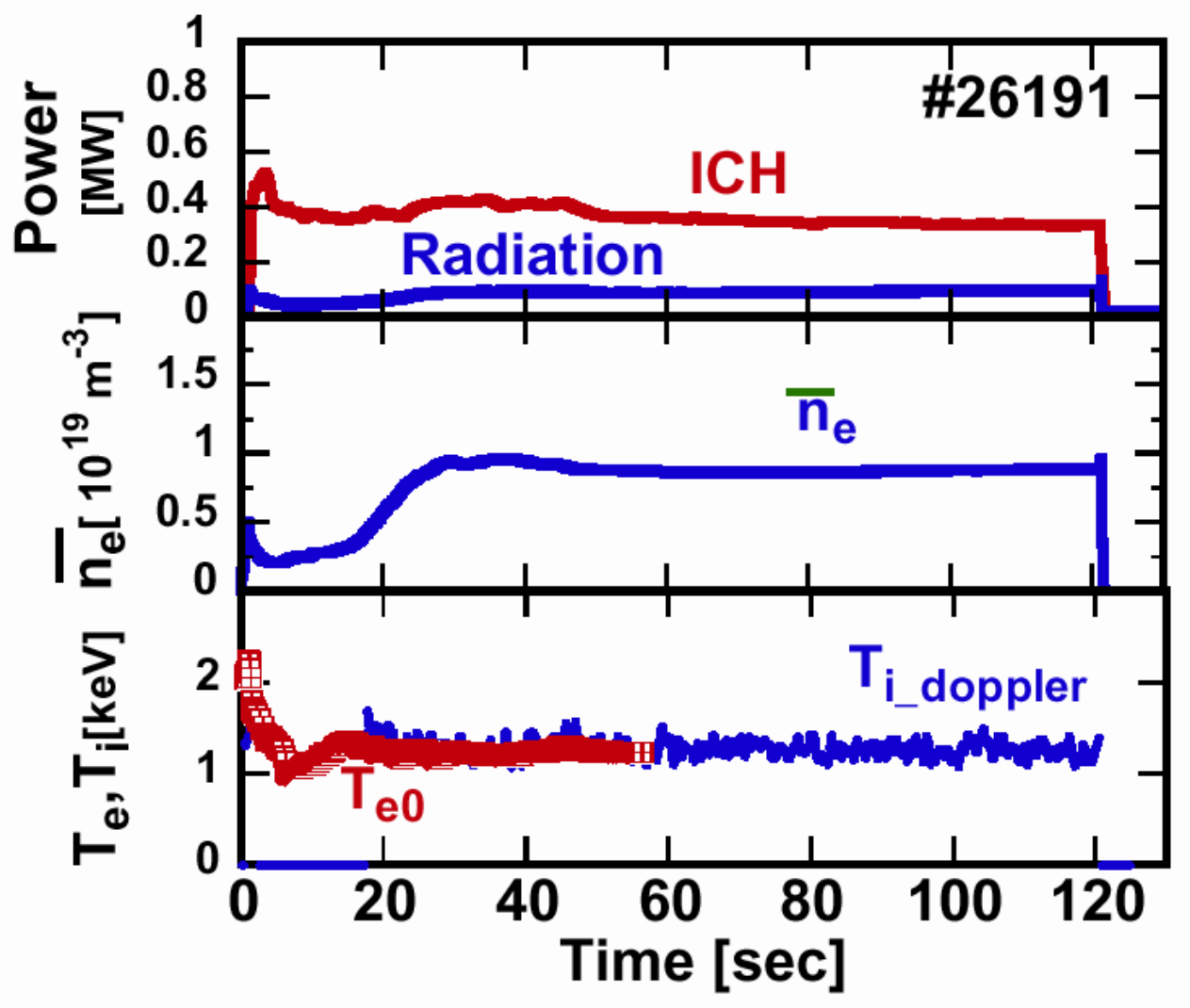

Fig.3.1a Long pulse LHD plasma [2] 
Energy distribution of minority protons at ICRH in LHD

- energetic protons are well confined

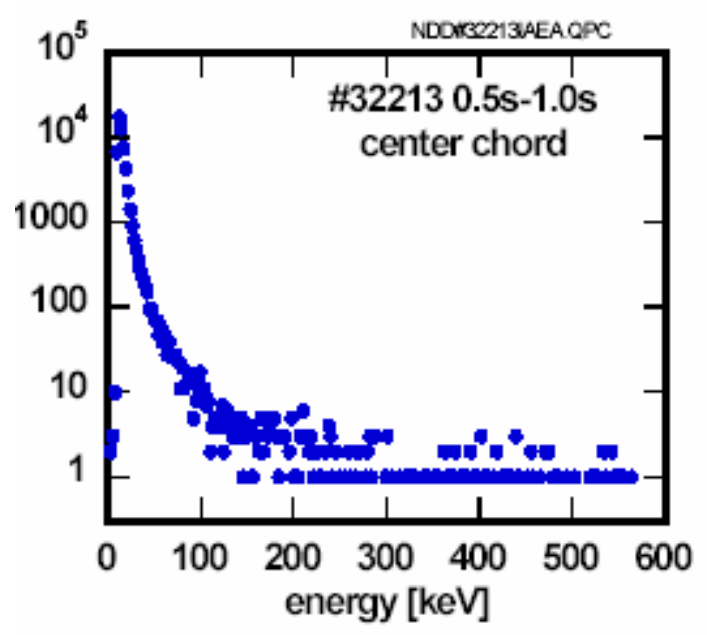

Fig.31b Protons Energy distribution [2]

4. ICRF Full Wave analysis in stellarators (PSTELION code)

$\downarrow \quad$ Motivation

Mainly ICRF heating - scenarios development

- optimised antennae

Also

- Low frequency instabilities (Alfen eigenmodes)

- Diagnostic (Ion Cyclotron Emission)

$\checkmark$ Features

- Configuration: 3D, 2D (tokamaks, mirrors included)

- Hot plasma model: Ion Bernstein Waves, Kinetic Alvfen 


\section{Waves, \\ Ion and electron FLR effects - through second order operators}

\section{Numerical method:}

- Finite difference, Modes expansion

- Magnetic flux coordinates

$\diamond$ Plasma equilibrium - VMEC (ORNL) code

The PSTELION code contour plots modeling of poloidal electric field in LHD with outside antenna is displayed in Fig.4a and magnetic surface averaged "radial" power deposition profile to protons - in Fig.4b. Power 1.6 MW is injected into $2 \mathrm{keV}(7 \% \mathrm{H}+\mathrm{He})$ plasma at a density $210^{19} \mathrm{~m}^{-3}$ with Zeff $=1.8$.

\section{Fast Waves in LHD propagate even at low plasma density - Helium plasma $+7 \%$ hydrogen}
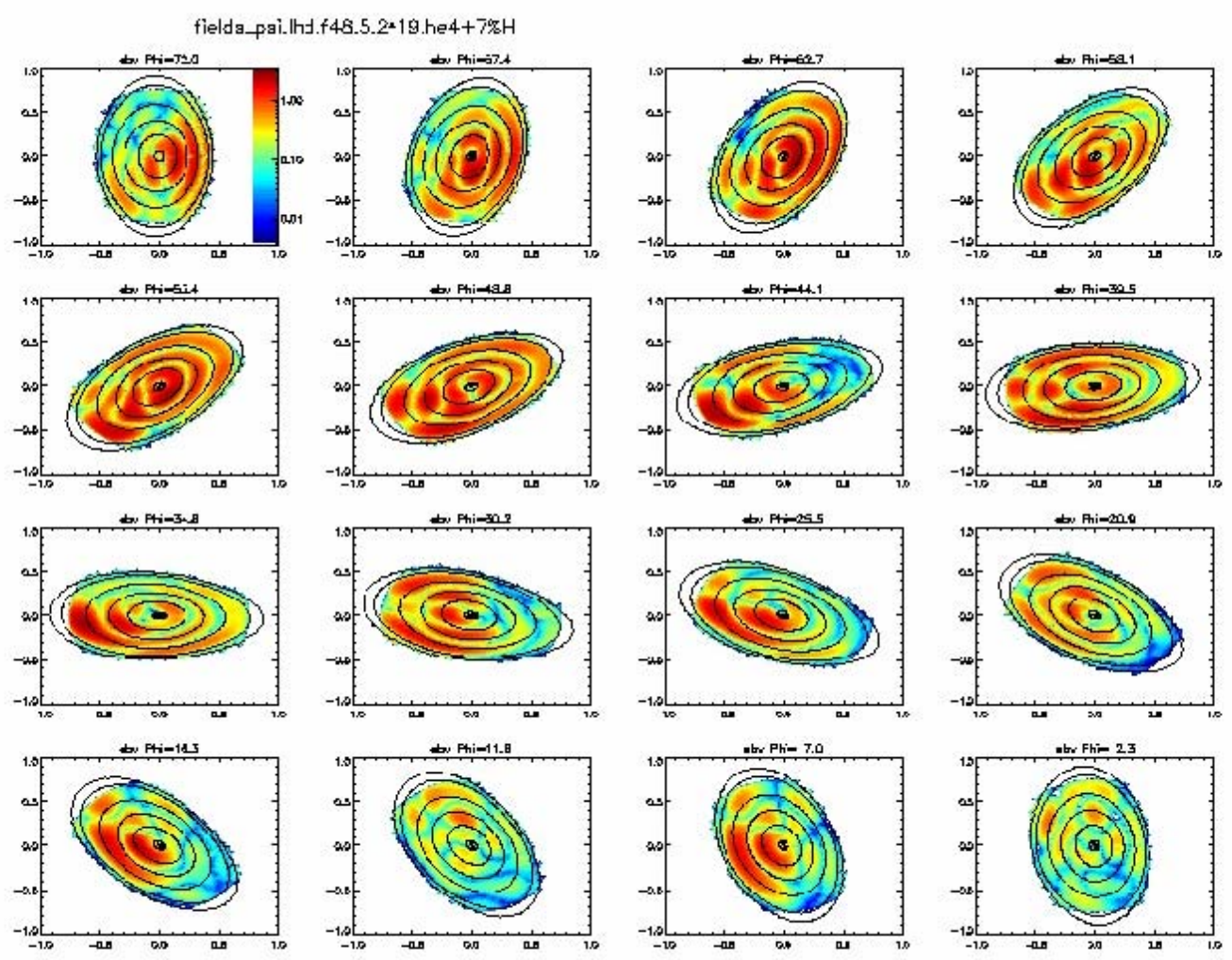


$$
\begin{aligned}
& \mathrm{N}_{\mathrm{e}}=210^{19} \mathrm{~m}^{-3} \\
& \mathrm{~T}_{\mathrm{e}, \mathrm{i}}=2 \mathrm{keV}
\end{aligned}
$$

Outside 4 loops antenna

Optical thickness $\tau=0.7$,

$P_{i}>P_{e}$, but

Energetic proton tail - F-P modelling - gives:

$<\mathrm{T}_{\text {eff }}>=20 \mathrm{keV}$

$\mathrm{P}_{\mathrm{RF}}=1.6 \mathrm{MW}$ redistributes part of the power to protons' drag on electrons:

$$
P_{e}=0.36 P_{R F}
$$

Power deposition to the protons is

radially reasonably peaked

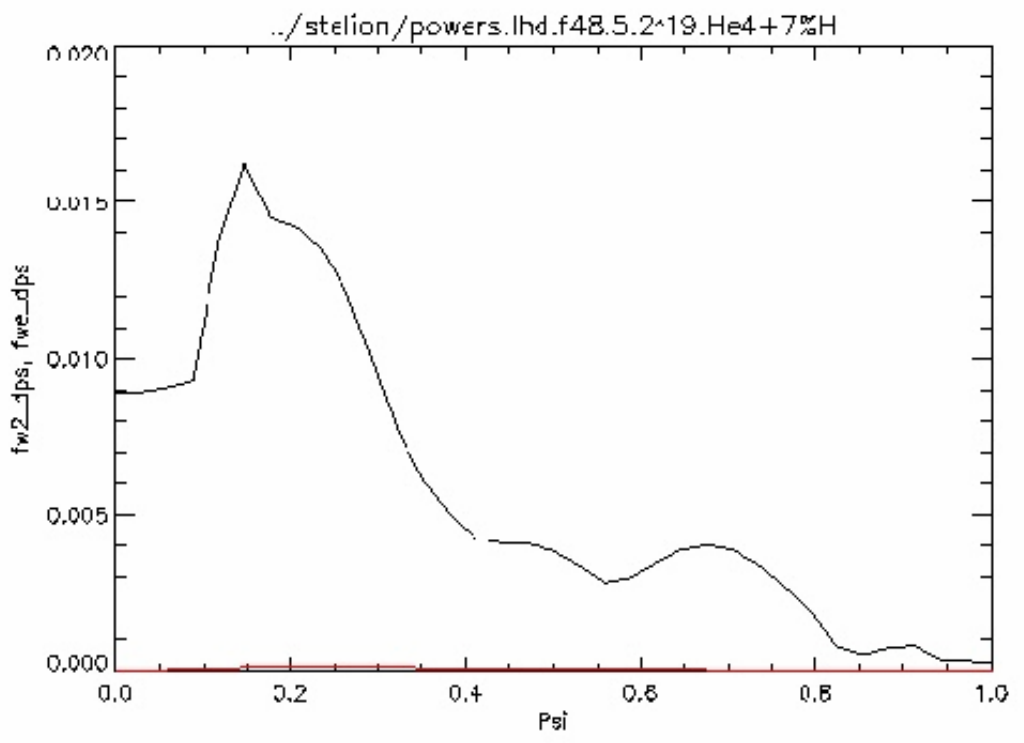


Example of $E_{\theta}$ distribution in LHD in hydrogen minority scenario

- $\mathrm{D} / \mathrm{He} 4(5 \% \mathrm{H})$ scenario

- single outside antenna

- high density $\mathrm{n}_{\mathrm{e} 0}=810^{19} \mathrm{~m}^{-3}$
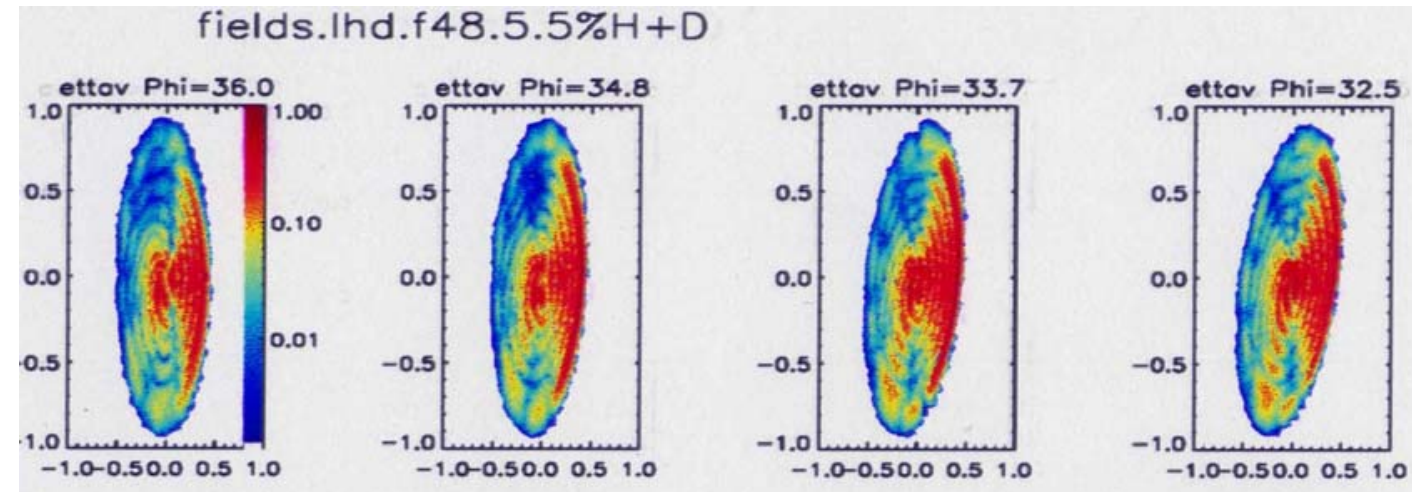

Fig.4c Poloidal electrical field in LHD hydrogen minority scenario 


\section{Power deposition to protons in LHD}

- is centrally peaked in $\mathrm{D} / \mathrm{He} 4(5 \% \mathrm{H})$

- is toroidally localised under antenna

$$
\Phi=0-4^{0}
$$

\section{fields.Ihd.f48.5.5\%H+D}
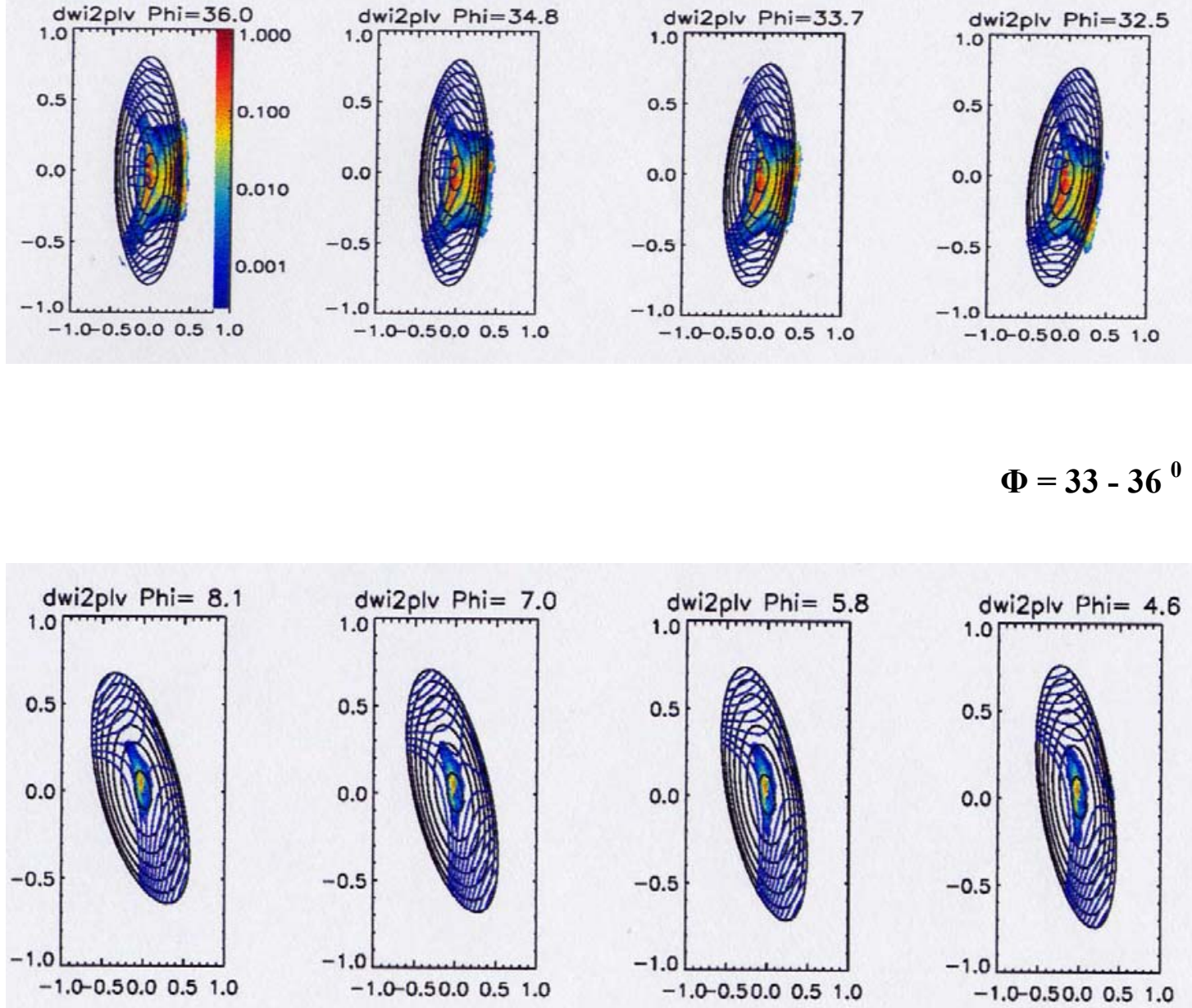

Fig.4d Power deposition to protons in LHD hydrogen minority scenario (overall toroidal machine angle)

\section{Magnetic Beach Heating scenario at W7-AS}




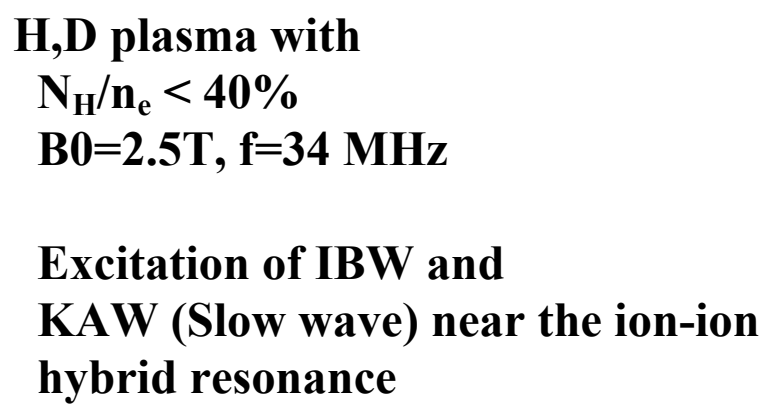

Heating of hydrogen and electrons. 


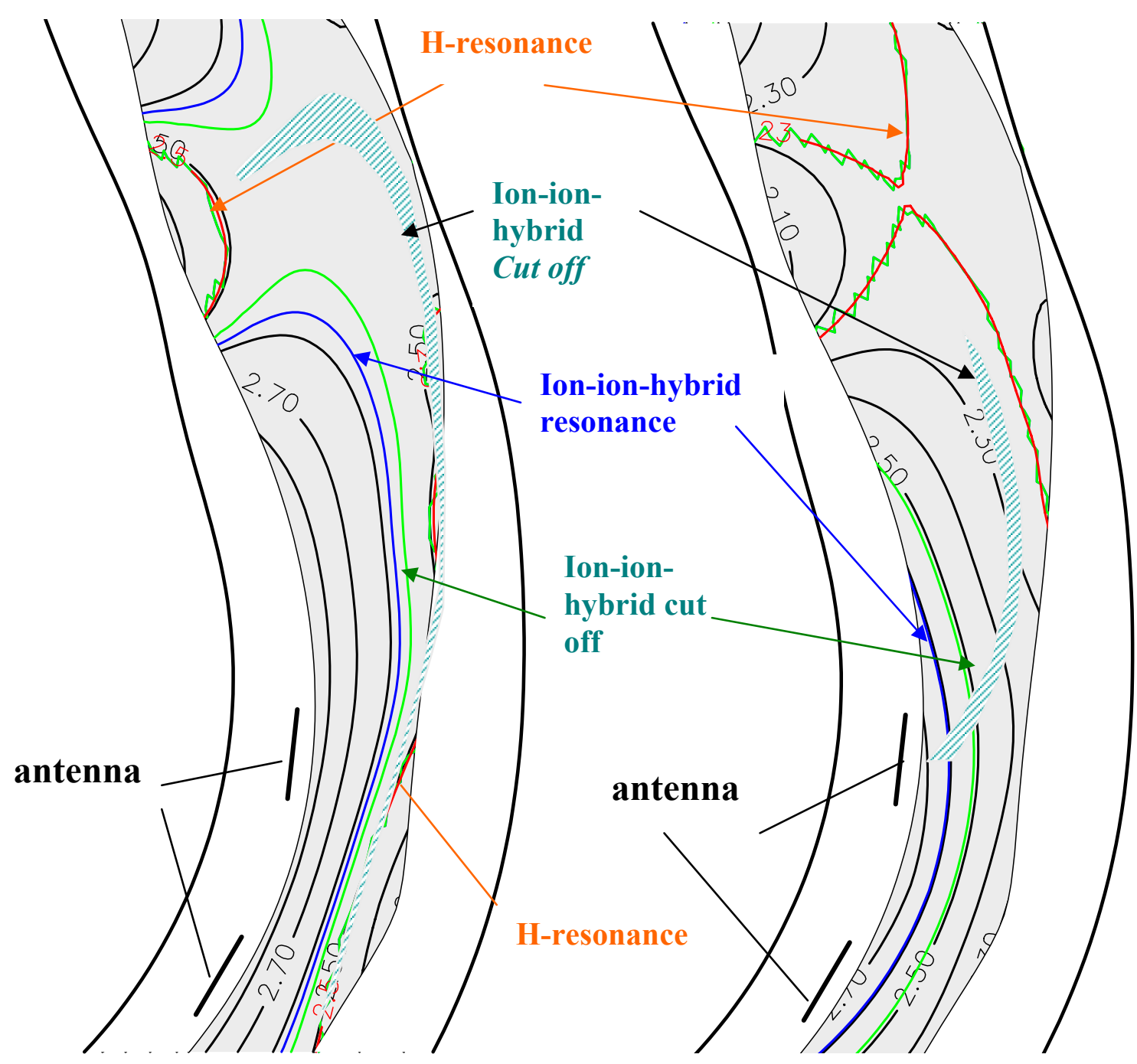

\section{Standart configuration}

Magnetic beach configuration

Fig.5.1 Resonances and cut off surfaces in standart and magnetic beach Configurations in W7-AS

The Quasi symmetric stellarator W7-AS permitted to explore the "magnetic beach" plasma heating scenario where IC resonance is located toroidally far away | from an antenna. The best plasma heating results with temperatures of $1 \mathrm{keV}$ were obtained in $\mathrm{H}, \mathrm{D}$ plasmas (with hydrogen concentration up to $40 \%$ ) if Both so called ion-ion hybrid resonance was in the plasma and the IC proton resonance occurred at the "beach". Dominant ion or electron heating depended on hydrogen concentration [4]. The heating efficiency was about $80 \%$ in this scheme. Minority scheme was also effective in W7-AS if the hydrogen concentration could be kept below $10 \%$ after chamber boronization. 


\section{In Two ion hybrid scenario in W7-AS}

mode conversion plays a dominant role

$$
\begin{aligned}
& \mathrm{F}=26.25 \mathrm{MHz}, \mathrm{N}_{\mathrm{e}}=810^{19} \mathrm{~m}^{-3} \\
& \mathrm{D}+20 \% \mathrm{H} \text { plasma } \\
& \text { Two loop inside antenna }
\end{aligned}
$$

\section{Radial electrical field $\mathbf{E}_{\psi}$}

(triangle cross section)

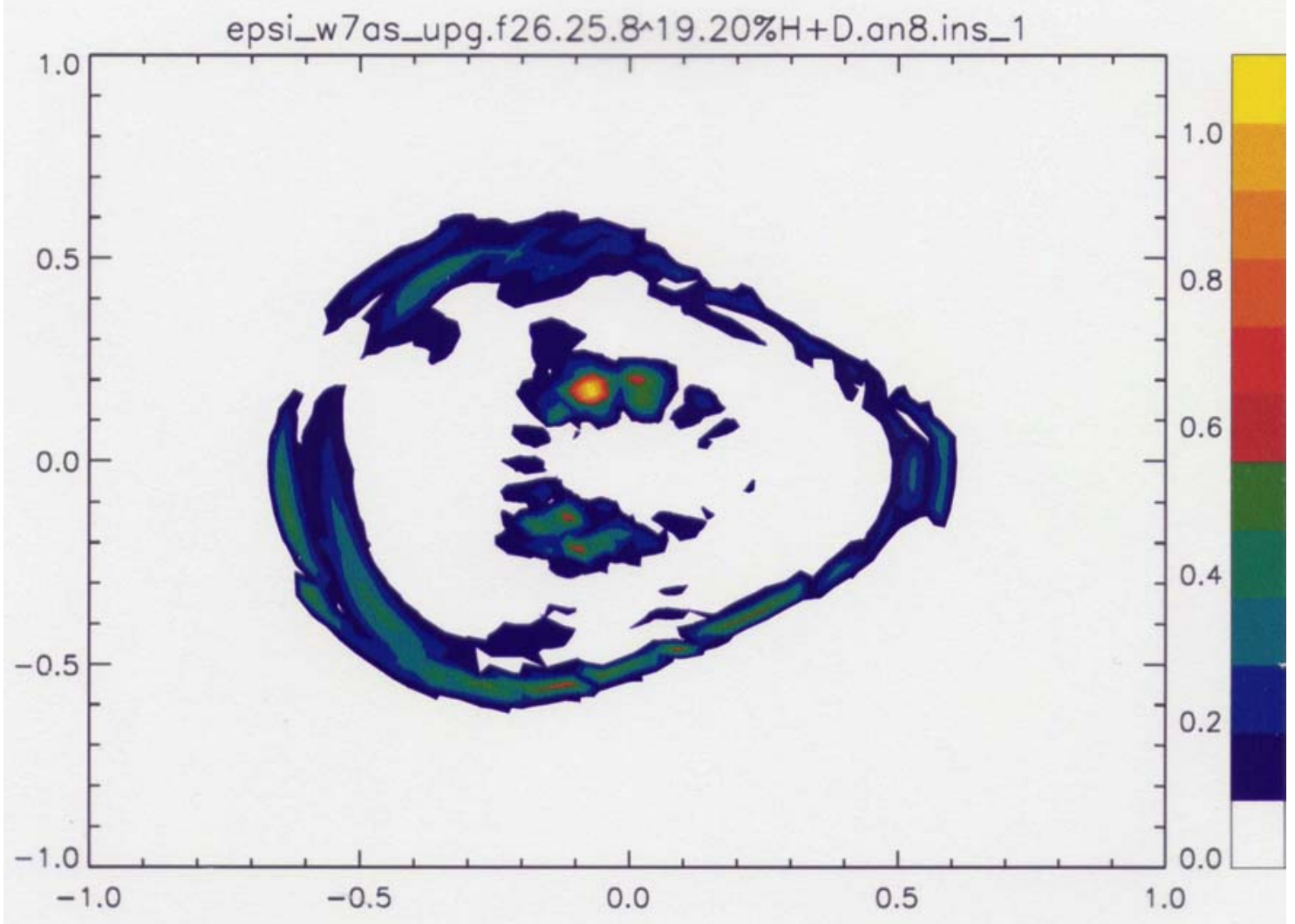

Fig.5.2 Contour plots of radial electric field abs $\left(\right.$ real $\left.\left(E_{\psi}\right)\right)$ in triangle $W 7-A S$ cross section in $D(20 \% H)$ magnetic beach scenario 


\section{Poloidal electrical field $\mathbf{E}_{\boldsymbol{\eta}}$}

(triangle cross section)

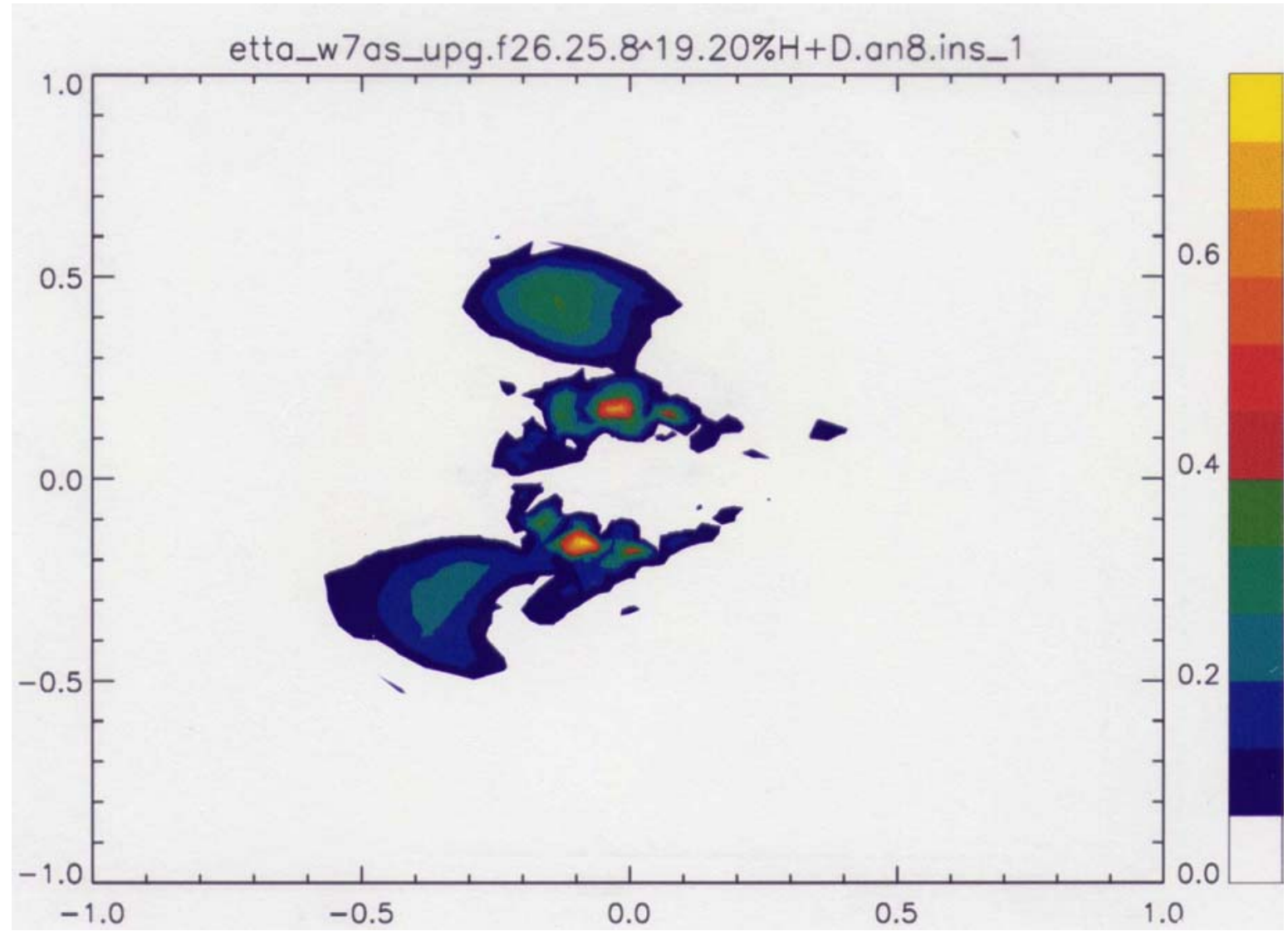

Fig.5.3 Contour plots of poloidall electric field abs $\left(\right.$ real $\left.\left(E_{\theta}\right)\right)$ in triangle W7-AS cross section in D(20\%H) magnetic beach scenario 


\section{Power deposition profile in W7-AS}

is reasonably peaked at ion-ion hybrid resonance

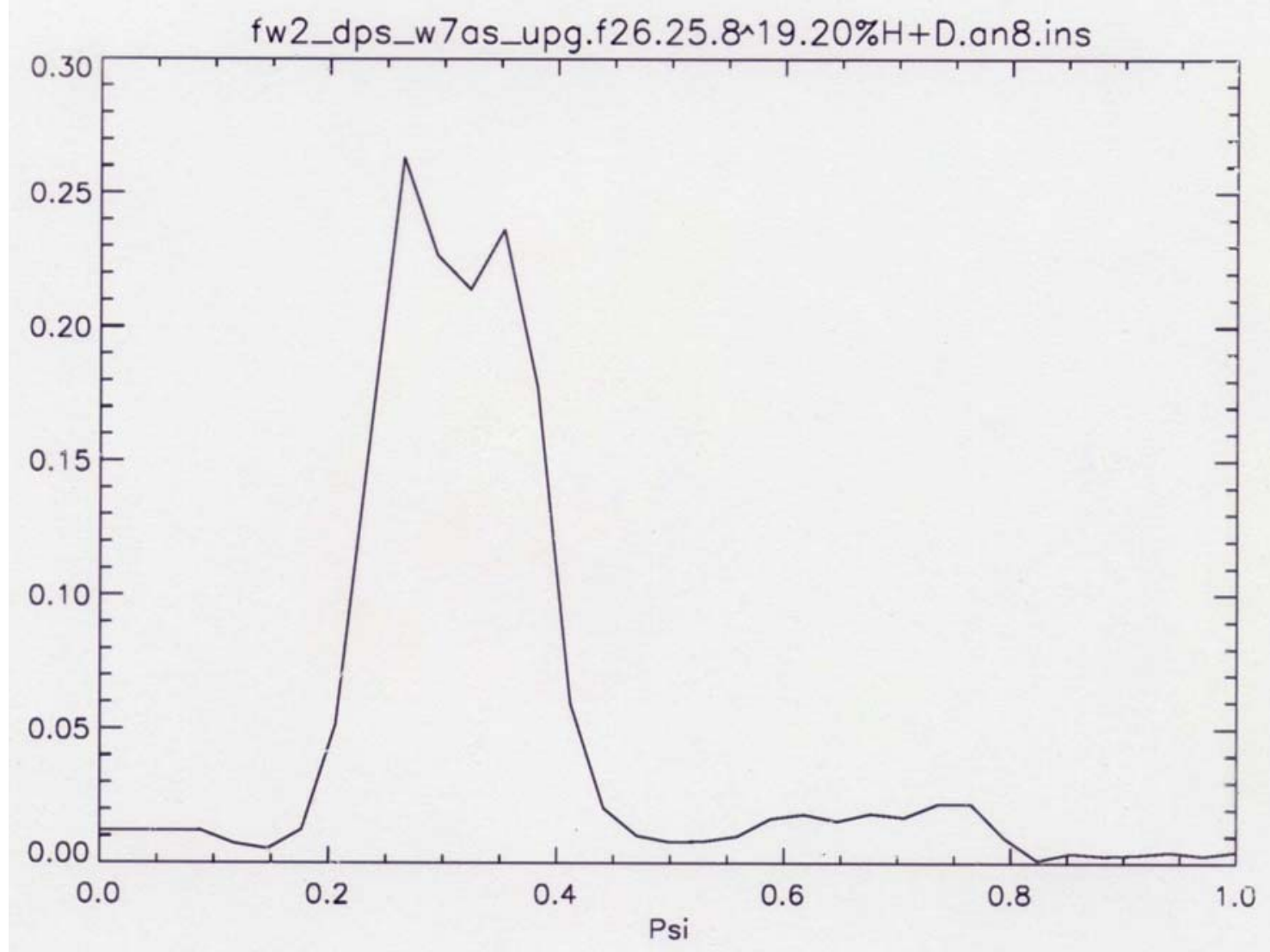

Fig.5.4 Power deposition to protons, W7-AS

3D code modelling shows (Figs.5.2-5.4) that mode conversion at high hydrogen content in beach scenario plays a crucial role. This is demonstrated by flux averaged well localised power deposition profile to protons in $20 \% \mathrm{H}+\mathrm{D}$ scenario with high field side antenna $(\mathrm{f}=26.2 \mathrm{MHz})$ 


\section{Summary D,H mode conversion in W7-AS}

Possible, if ion-ion hybrid resonance is located inside of plasma

Good heating efficiency

No degradation with power observed.

Absorbed power density has maximum near the ion-ion hybrid resonance.

Overthermal hydrogen observed for high electron temperatures

6. W7-X modelling plasma parameters (+VMEC equilibrium)

$$
\begin{aligned}
& \mathrm{B}_{0}=2.5-3.0 \mathrm{~T} \\
& \mathrm{n}_{\mathrm{e} 0}=(2-8) 10^{19} \mathrm{~m}^{-3} \\
& \mathrm{~T}_{\mathrm{e} 0}=2-8 \mathrm{keV} \\
& \mathrm{T}_{\mathrm{i} 0}=2-8 \mathrm{keV} \\
& \mathrm{T}_{\mathrm{i} 0}(\text { minority })=27 \mathrm{keV} \\
& \mathrm{n}_{\mathrm{e}}=\left(\mathrm{n}_{\mathrm{e} 0}-\mathrm{n}_{\mathrm{a}}\right)\left(1-\mathrm{s}^{2}\right)_{\mathrm{n}}^{\alpha}+\mathrm{n}_{\mathrm{a}}, \\
& { }_{\mathrm{n}}^{\alpha}=0.15 \\
& \mathrm{n}_{\mathrm{e}}(\mathrm{s})=\mathrm{n}_{\mathrm{a}} \exp (-(\mathrm{s}-\mathrm{a}) / \Delta), \\
& \mathrm{T}_{\mathrm{e}}(\mathrm{s})=\left(\mathrm{T}_{\mathrm{e} 0}-\mathrm{T}_{\mathrm{a}}\right)\left(1-\mathrm{s}^{2}\right)_{\mathrm{T}}^{\alpha}+\mathrm{T}_{\mathrm{a}}, \quad \mathrm{s} \leq \mathrm{a}_{\text {Lim }} \\
& { }_{\mathrm{T}}^{\alpha}=1
\end{aligned}
$$




$$
\mathrm{T}(\mathrm{s})=\mathrm{T}_{\mathrm{a}}, \quad \mathrm{T}_{\mathrm{a}}=100-300 \mathrm{eV}, \quad \mathrm{s}>\mathrm{a}_{\mathrm{Lim}}
$$

Multi loop outside antenna

(in bean plasma section mainly)
Number of loops,
$\mathrm{N}_{\mathrm{c}}=2-8$
Toroidal loop width,
$\mathrm{D}=0.2 \mathrm{~m}$
Period of loops
$\mathrm{T}=0.33 \mathrm{~m}$
Phase difference between loops
$\Phi=90^{\circ}$
Antenna plasma distance
$0.05 \mathrm{~m}$
Antenna - conducting wall distance $0.02-0.1 \mathrm{~m}$ Poloidal antenna extent $\quad \pm 60^{\circ}$

\section{Poloidal electrical fields in W7-X}

\section{Magnetic beach scenario}

\footnotetext{
- 3\%He-3 in D-plasma

- $\mathrm{f}=24.6 \mathrm{MHz}$

4 loops outside

antenna

$\mathrm{n}_{\mathrm{e}}=810^{19} \mathrm{~m}^{-3}$
$\mathrm{~T}_{\mathrm{e} 0}=3 \mathrm{keV}$
$\mathrm{T}_{\mathrm{i} 0}=5 \mathrm{keV}$
$\mathrm{T}_{\mathrm{He} 3}=22 \mathrm{keV}$
}

\section{Projections for W7-X stellarator.}

$\mathrm{W} 7-\mathrm{X}$ is a five fold modular stellarator with a major radius $\mathrm{R}=5.6 \mathrm{~m}$ and an averaged minor radius $<\mathrm{a}>=0.5 \mathrm{~m}$. The typical magnetic field on axis will 2.5$3.0 \mathrm{~T}$. In this large machine the fast waves can propagate even at low densities. For poor wave absorption the plasma behaves like a multi-mode resonator. Using 
PSTELION different heating scenarios different heating scenarios have been investigated. Fig.6.1 shows the poloidal wave electric field for the minority $\mathrm{D}(3 \% \mathrm{He} 3)$ beach scenario with a LFS four-loop antenna at different toroidal coss-sections within one module $\left(\mathrm{N}_{\mathrm{e}}=810^{19} \mathrm{~m}^{-3}, \mathrm{~T}_{\mathrm{e}}=3 \mathrm{keV}, \mathrm{T}_{\mathrm{i}}=5 \mathrm{keV}\right.$, mirror ratio 0.8 ).
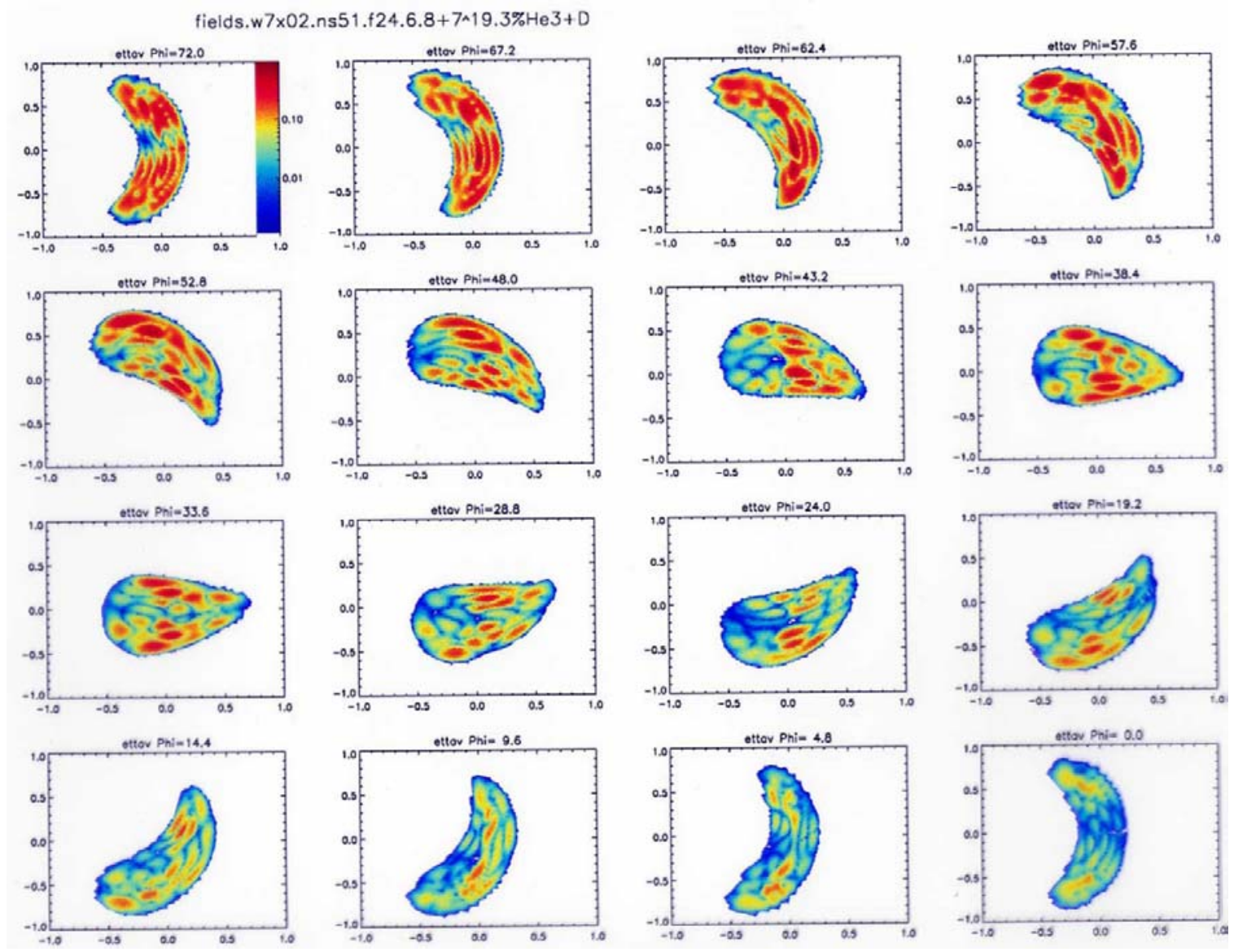

Fig.6.1 Poloidal electrical field distribution along one toroidal module in $\mathrm{W7}-\mathrm{X} \mathrm{D}(\mathrm{He} 3)$ scenario at $24.6 \mathrm{MHz}$.

\section{Magnetic beach $\omega=2 \omega_{\mathrm{cD}}$ in $\mathrm{W} 7-\mathrm{X}$}

Fig.6.2 shows the poloidal RF electric field for the second harmonic beach scenario with a LFS four-loop antenna at different toroidal cross-sections one half-module $\left(\mathrm{N}_{\mathrm{e}}=210^{19} \mathrm{~m}^{-3}, \mathrm{f}=36.8 \mathrm{MHz}, \mathrm{T}_{\mathrm{e}}=3 \mathrm{keV}, \mathrm{T}_{\mathrm{i}}=5 \mathrm{keV}\right.$, mirror ratio 0.8$)$. 

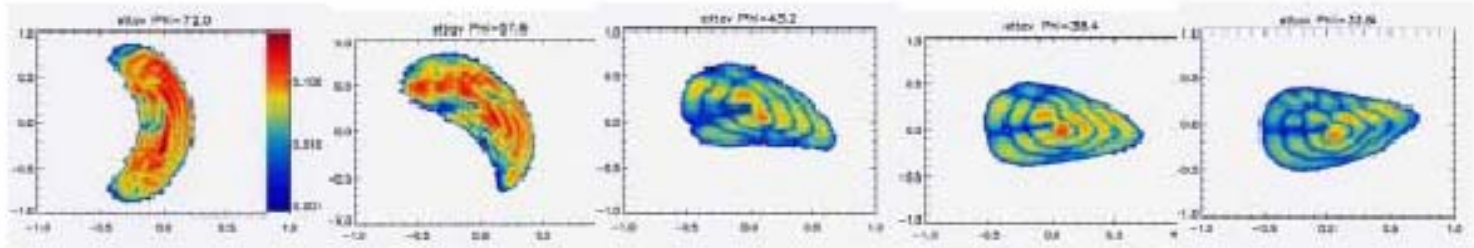

Fig.6.2 Poloidal electrical field in $W 7-X 2 \omega_{c D}$ scenario $(f=36.8 \mathrm{MHz})$.

Fig.6.3 shows the power absorbed by the deuterons. Power deposition in beach area is centrally peaked. Estimates of the absorbed power on the RF field amplitude predict that about $95 \%$ of the RF power during one pass throudh the beach region. Not absorbed RF power will be absorbed at the next several toroidal beaches.

\section{well peaked ion power deposition}
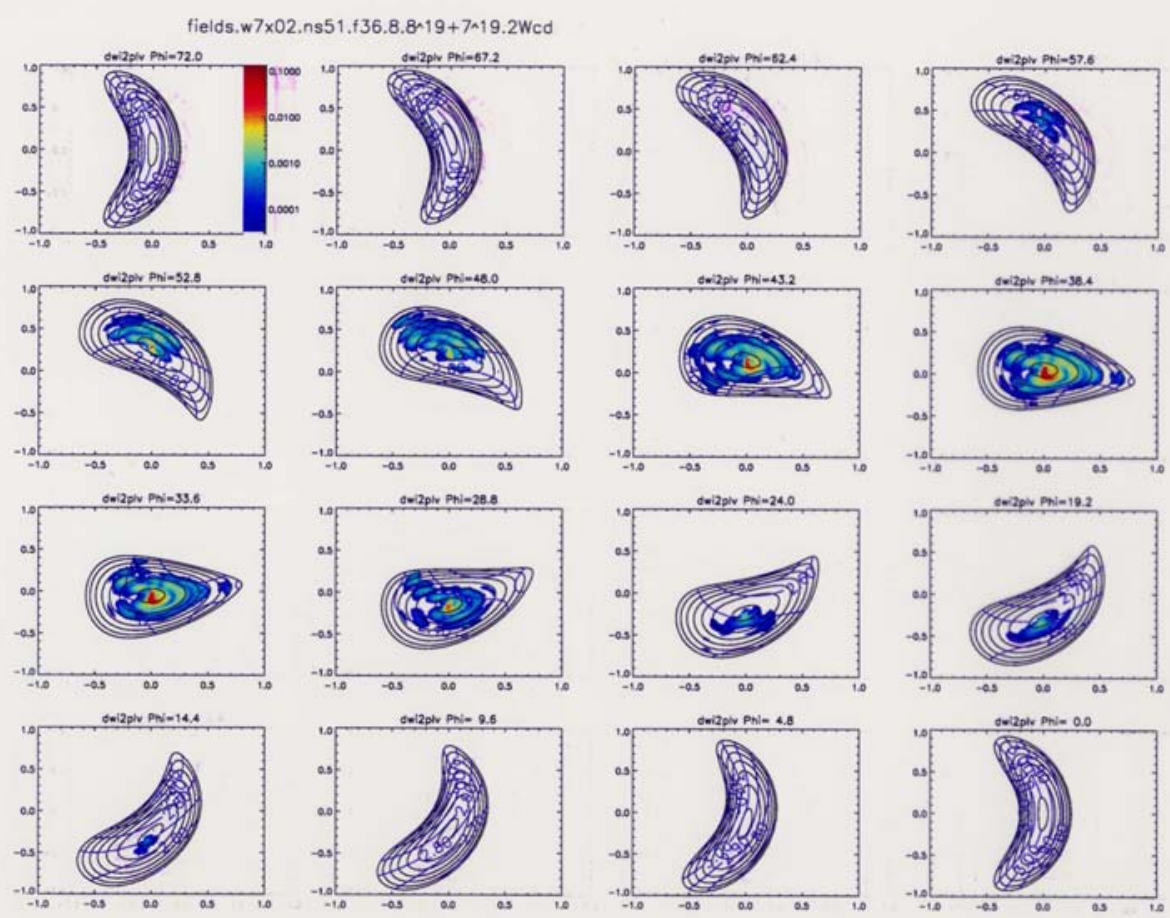

Fig.6.3 Power deposition to deuterons in W7-X at $36.8 \mathrm{MHz}\left(\omega=2 \omega_{c D}\right)$ 


\section{Tokamak-like minority ions scenario in W7-X}

Tokamak-like scenarios (resonance in front of the antenna antenna) are also possible at $\mathrm{W} 7-\mathrm{X}$.

$$
\begin{aligned}
& \text { - resonance under antenna } \\
& \text { - power deposition to protons } \\
& 5 \% \mathrm{H}+\mathrm{D} \\
& \mathrm{F}=45.5 \mathrm{MHz} \\
& \mathrm{N}_{\mathrm{e}}=810^{19} \mathrm{~m}^{-3} \\
& \mathrm{P}_{\mathrm{i}}>>\mathrm{P}_{\mathrm{e}}
\end{aligned}
$$

Power deposition to protons in $5 \% \mathrm{H}+\mathrm{D}$ plasma is shown by Fig. 6.4 for above plasma parameters and frequency $\mathrm{f}=45.5 \mathrm{MHz}$. 

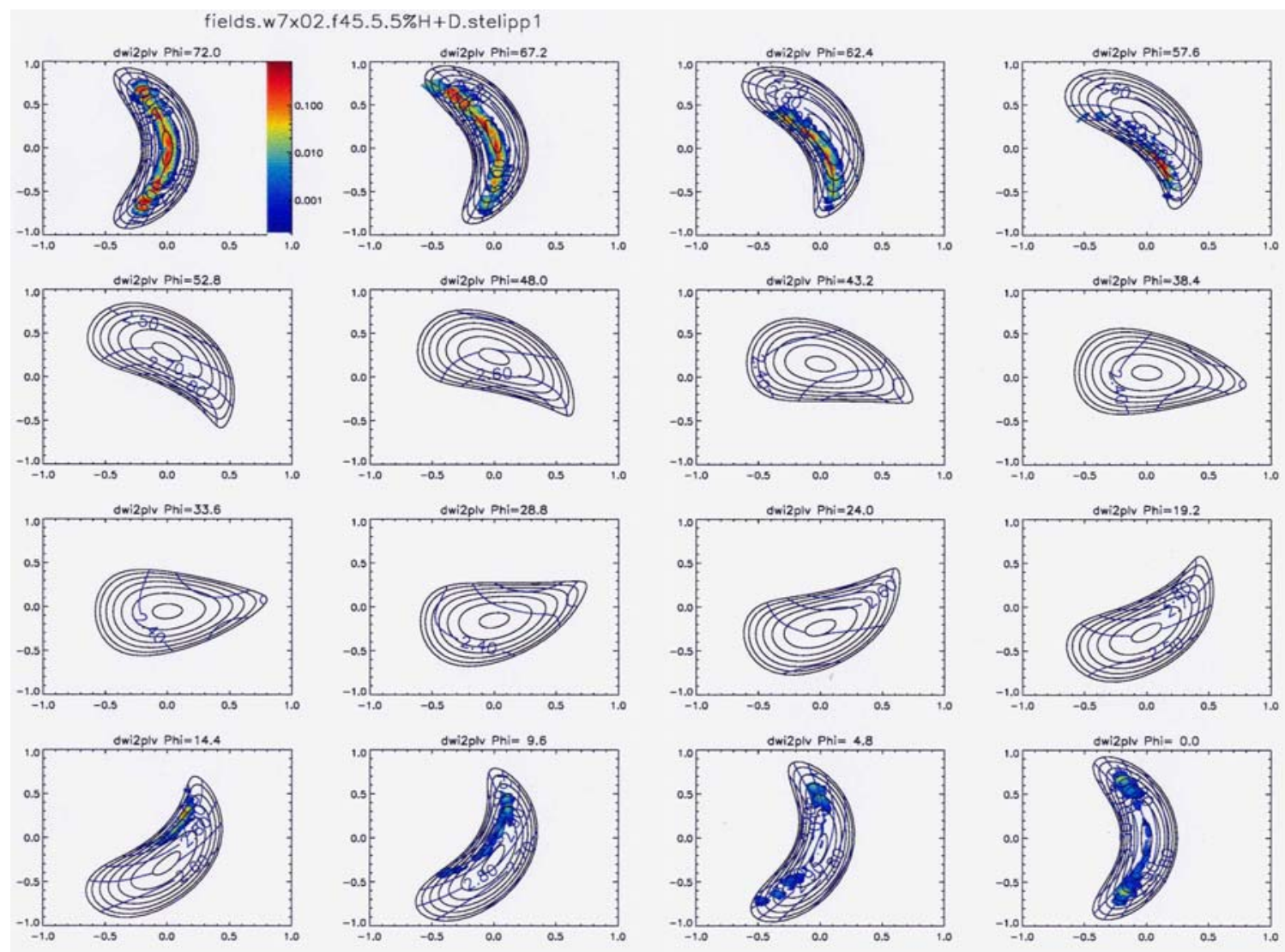

Fig. 6. 4 Power deposition to protons in one module for $5 \% H+D$ scenario.

The toroidally averaged power deposition profile is reasonably peaked. Other scenarios with good heating efficiencies include two-ion hybrid and second harmonic hydrogen heating. Outside antenna is appropriate one and is projected to be moveable one [4]. Second hydrogen harmonic scenario is accounted by RF generator upper frequency $75 \mathrm{MHz}$. Finally, several scenarios provide in W7_X reliable strong absorption mechanisms with a central power deposition. They provide dominant ion or electron heating in the machine.

\section{Pure electron heating regime in $\mathrm{W} 7-\mathrm{X}$}

This scenario, to be efficient, requires sufficientlylarge electron beta, that is provided by ECR plasma preheating by W7-X gyrotron complex. Poloidal electric field is displayed by Fig. 6.5. One can see that Fast Waves completely occupy the toroidal multi mode plasma resonator.

- no IC resonance in plasma

- multi mode FW excitation 


$$
\begin{aligned}
& \mathrm{F}=52 \mathrm{MHz} \\
& \mathrm{N}_{\mathrm{e}}=210^{20} \mathrm{~m}^{-3} \\
& \mathrm{~T}_{\mathrm{e}}=9.8 \mathrm{keV} \\
& \text { ICRH }+\mathrm{ECRH}
\end{aligned}
$$
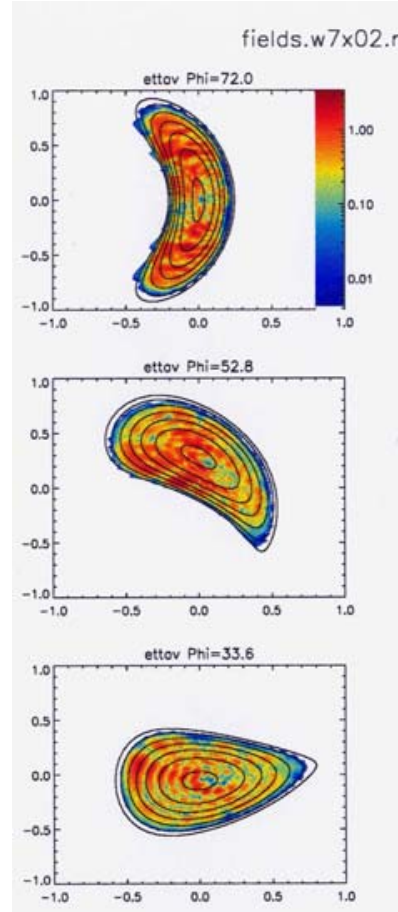

ettov Phi 14.4

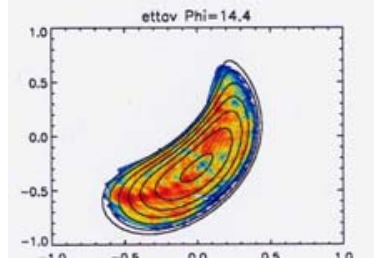

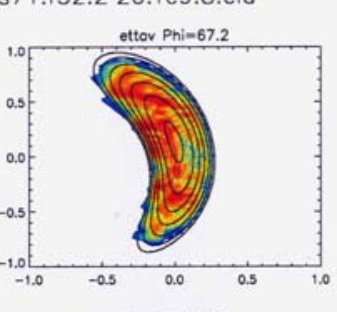
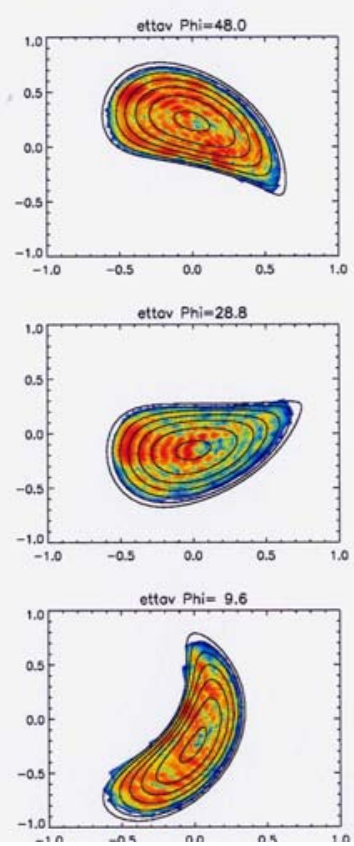

ettov Phi 62.4
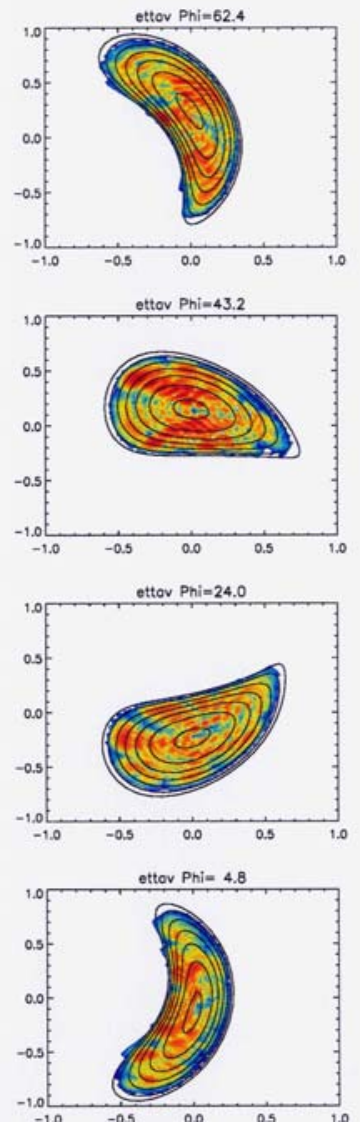

ettov Phi=57.6

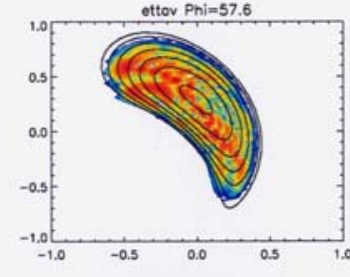

ettov Phi $=38.4$

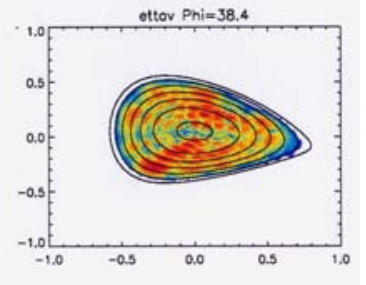

ettov Phi= 19.2
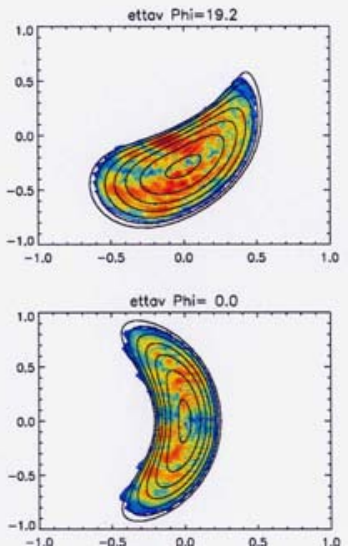

Fig. 6.5 Electrical poloidal fields distribution at pure electron heating regime in $\mathrm{W7}-\mathrm{X}$ at frequency $52 \mathrm{MHz}$.

\section{Summary:}

W7-AS: 
- Standard scenarios on tokamaks (second harmonic, minority, mode-conversion heating) are also successful in a stellarator.

- Strong dependence on minority concentration.

- $\quad$ Trapped particle loss may contribute to reduced heating efficiency at high power

- Magnetic beach modelling supports importance of mode conversion (ion / electron heating partitioning)

\section{LHD:}

- Ion heating generally dominant

- Heating efficiency comparable to NBI

- Dependence on configuration and minority concentration

- Discovering of efficient fundamental harmonic plasma heating

- Reasonable modelling predictions in minority scenario W7-X:

- Tokamak and magnetic beach scenarios are looking favourable ones on a basis of full wave ICRF code. Antenna location is unimportant: so technically more simple outside antennae can be used. 


\section{Projections for NCSX}

This 3-period high beta MHD stable stellarator (a quasi axial-symmetric machine) simultaneously explores advantages of tokamaks and stellarators [5]. Second harmonic scenario is of primary interest for NCSX due to projected high plasma beta. Outside antenna and its matching elements are easily located between machine toroidal coils in bean shaped plasma cross section (Fig. 7.1). Poloidal electric field distribution for hydrogen plasma is given in Fig.7.2 $\left(<\mathrm{N}_{\mathrm{e}}>\right.$ $=510^{19} \mathrm{~m}^{-3},<\mathrm{T}_{\mathrm{i}}>=1.9 \mathrm{keV},\left\langle\mathrm{T}_{\mathrm{e}}>=1.14 \mathrm{keV}, \mathrm{f}=45.4 \mathrm{MHz}, \mathrm{Bo}=1.7 \mathrm{~T}\right.$ ) and shows easy fast wave (FW) propagation from Low Field Side two loop antenna.

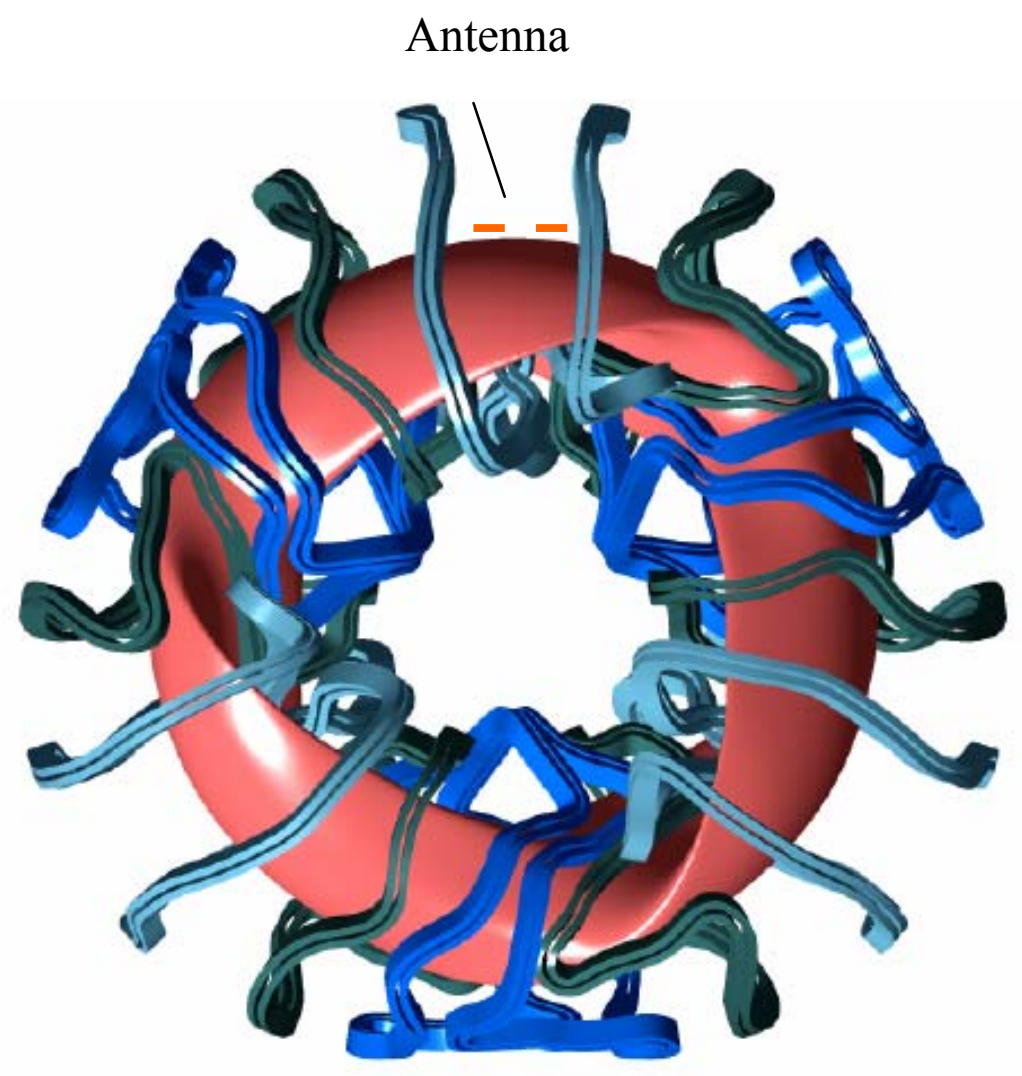

Fig.7.1 Outside antenna location in NCSX machine. 


\section{Electrical field $\mathrm{E}_{\theta}$ in NCSX}

$\mathrm{f}=45.4 \mathrm{MHz}\left(2 \mathrm{~W}_{\mathrm{cH}}\right), 2$ loop antenna
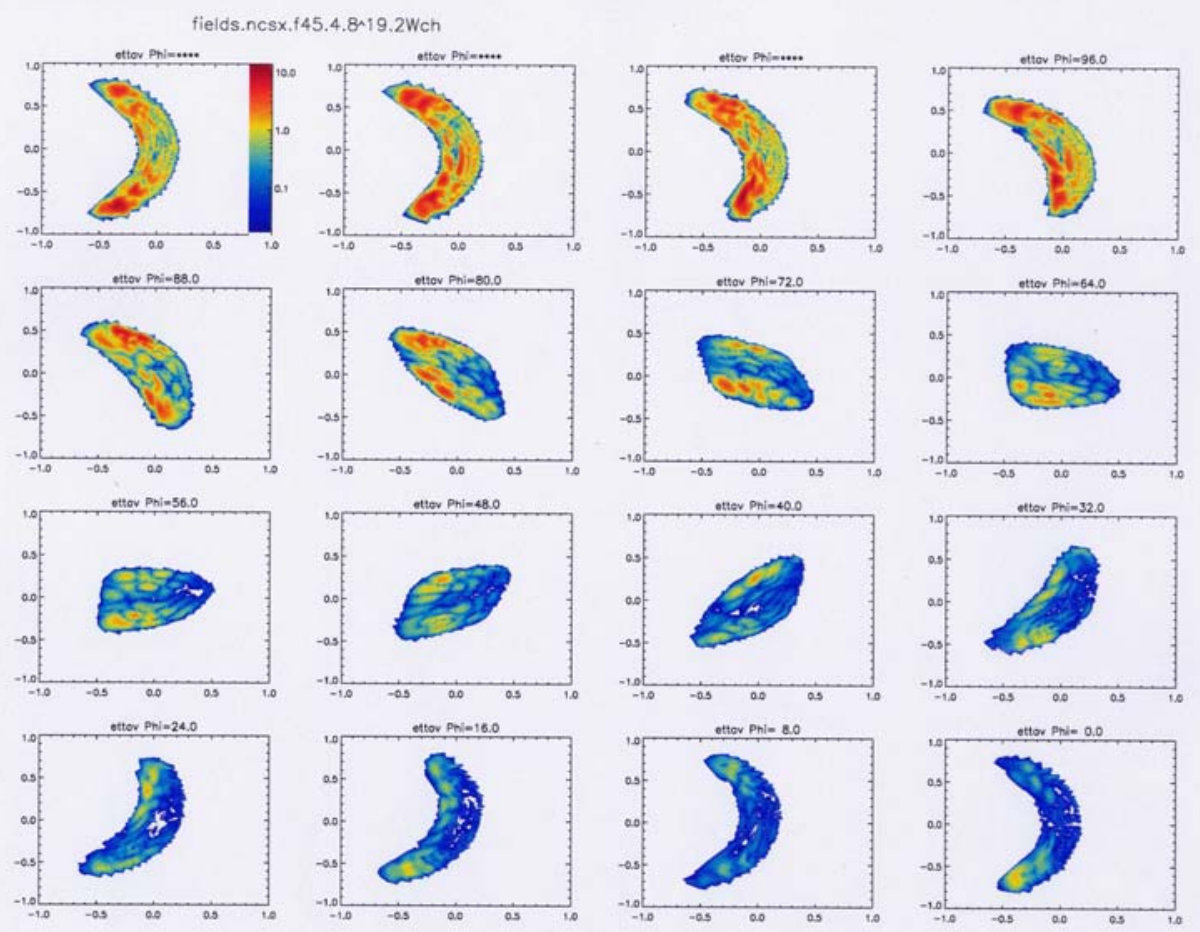

Fig.7.2 Abs $\left(\operatorname{real}\left(E_{\theta}\right)\right)$ distribution in NCSX, $2 \omega_{c H}(f=45.4 \mathrm{MHz}$, two loop out phase outside antenna in bean shape section).

2D Contour plots of power deposition to the protons are displayed in Fig.7.3 and show qualitatively centrally peaked ones. 


\section{Radial power deposition profile in NCSX}

$$
\begin{aligned}
-2 \omega_{\mathrm{cH}} \text { scenario: } \mathrm{f}=45.4 \mathrm{MHz} \\
<\mathrm{n}_{\mathrm{e}}>=510^{19} \mathrm{~m}^{-3} \\
<\mathrm{T}_{\mathrm{e}}>=1.14 \mathrm{keV} \\
<\mathrm{T}_{\mathrm{i}}>=1.9 \mathrm{keV} \\
\mathrm{P}_{\mathrm{i}}=0.98 \mathrm{P}_{\mathrm{RF}}
\end{aligned}
$$

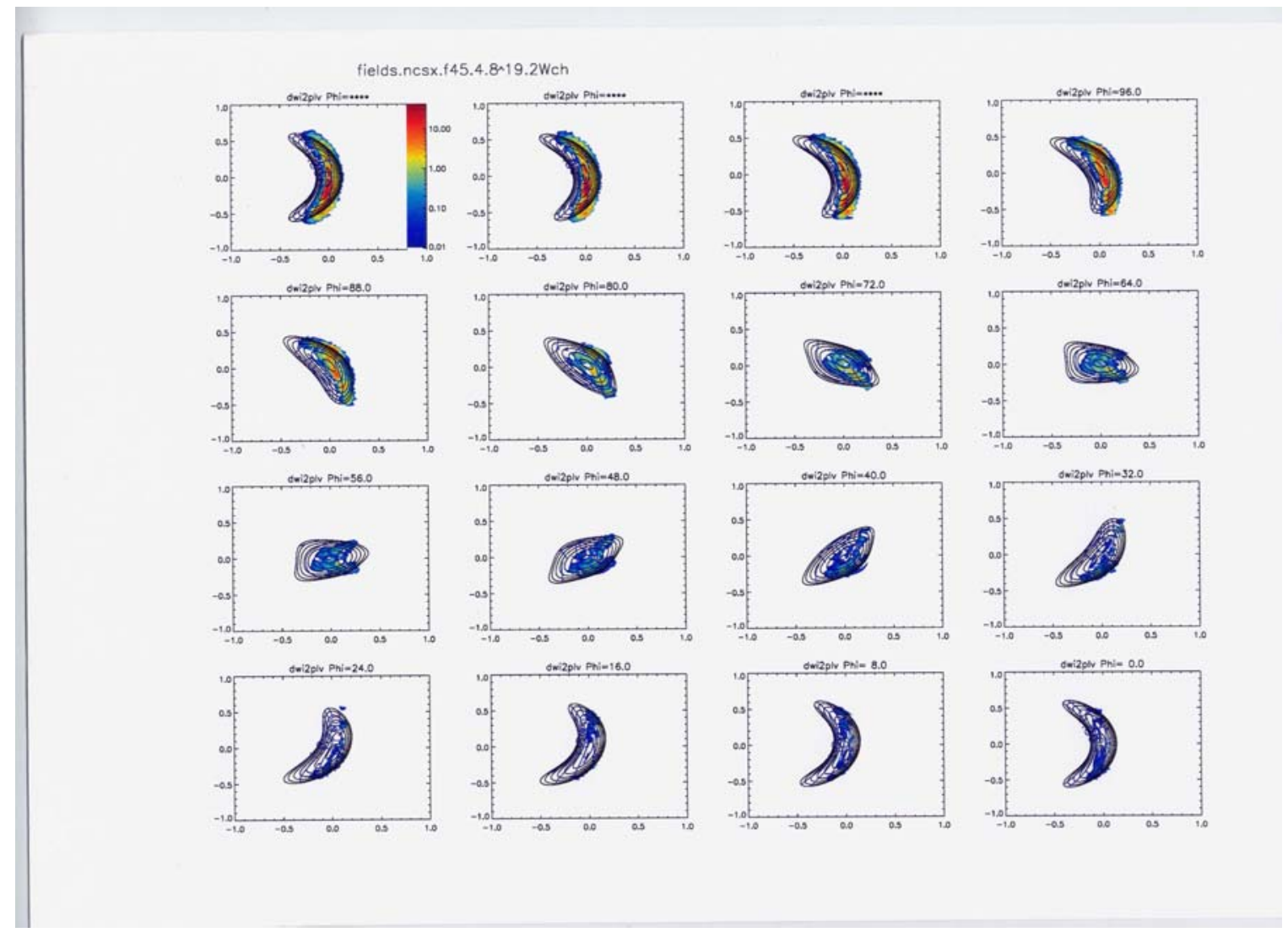

Fig.7.3 Power deposition to protons along NCSX torus, $2 \omega_{c H}$.

Integrated over magnetic surfaces tegrat over magnetic surface radial power deposition to protons is shown in Fig.7.4. 
One can see the FW radial structure with reasonably centrally peaked power deposition. Figs.7.3, 7.4 show that NCSX operates in a tokamak-like scenario - with the IC resonance in front of the antenna. Up to $98 \%$ of RF power is absorbed by the ions.

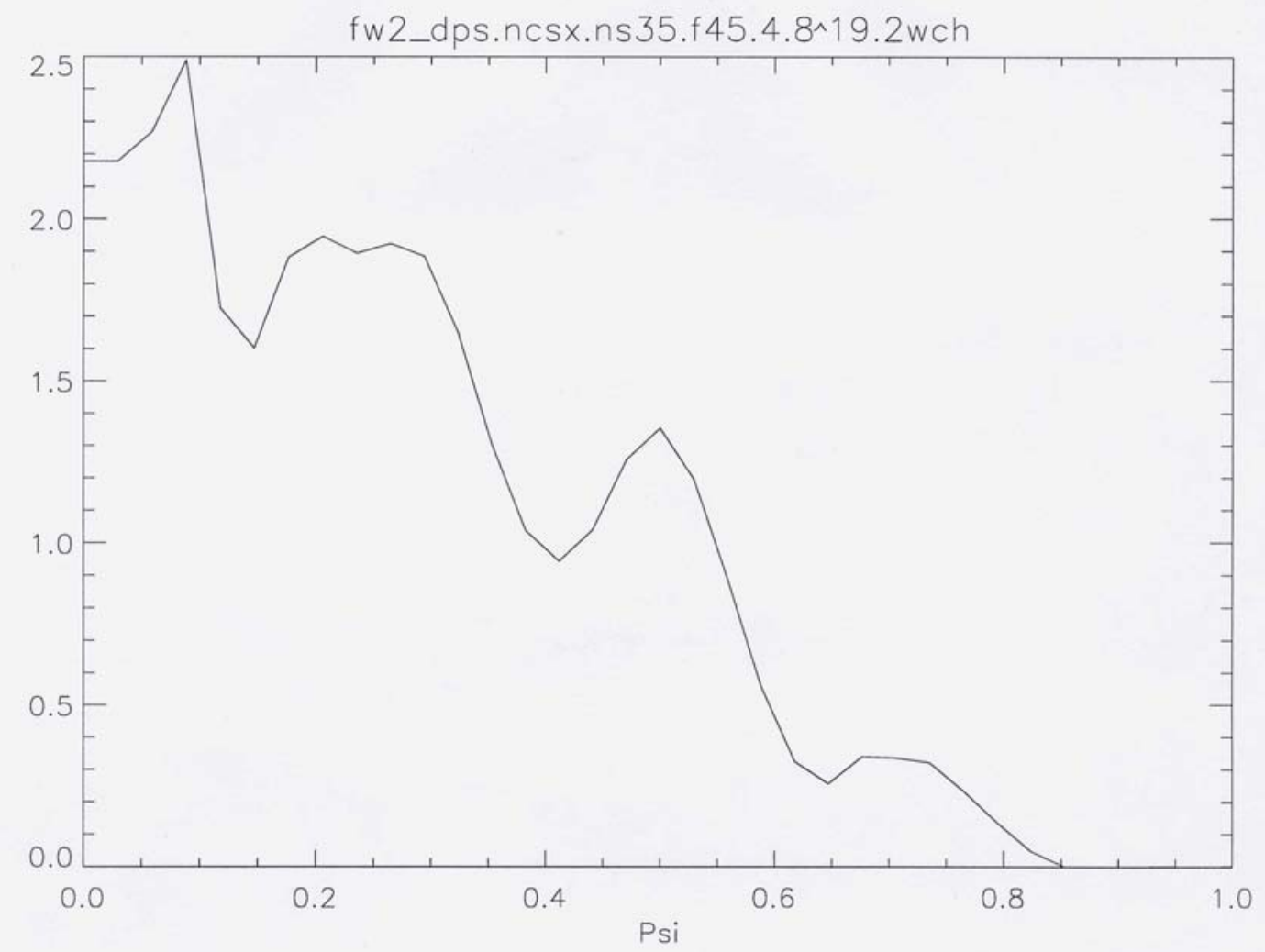

Fig.7.4 Radial power deposition to the protons in NCSX, $2 \omega_{c H}$ 
In the minority ions scenario $5 \% \mathrm{H}$ in $\mathrm{D} / \mathrm{He} 4, \mathrm{f}=23.5 \mathrm{MHz}$, about $70 \%$ of the $\mathrm{RF}$ power is absorbed by protons and $20 \%$ by deuterons, the rest of power goes to the electrons.

Radial power deposition to minority protons, presented in Fig.7.5, is excellent centrally peaked one. Power deposition to the electrons is given by Fig.7.6 and discovers a possibility for driven RF current creation (by appropriate antenna's loops phasing), that may be useful for modification of shear profile in NCSX.

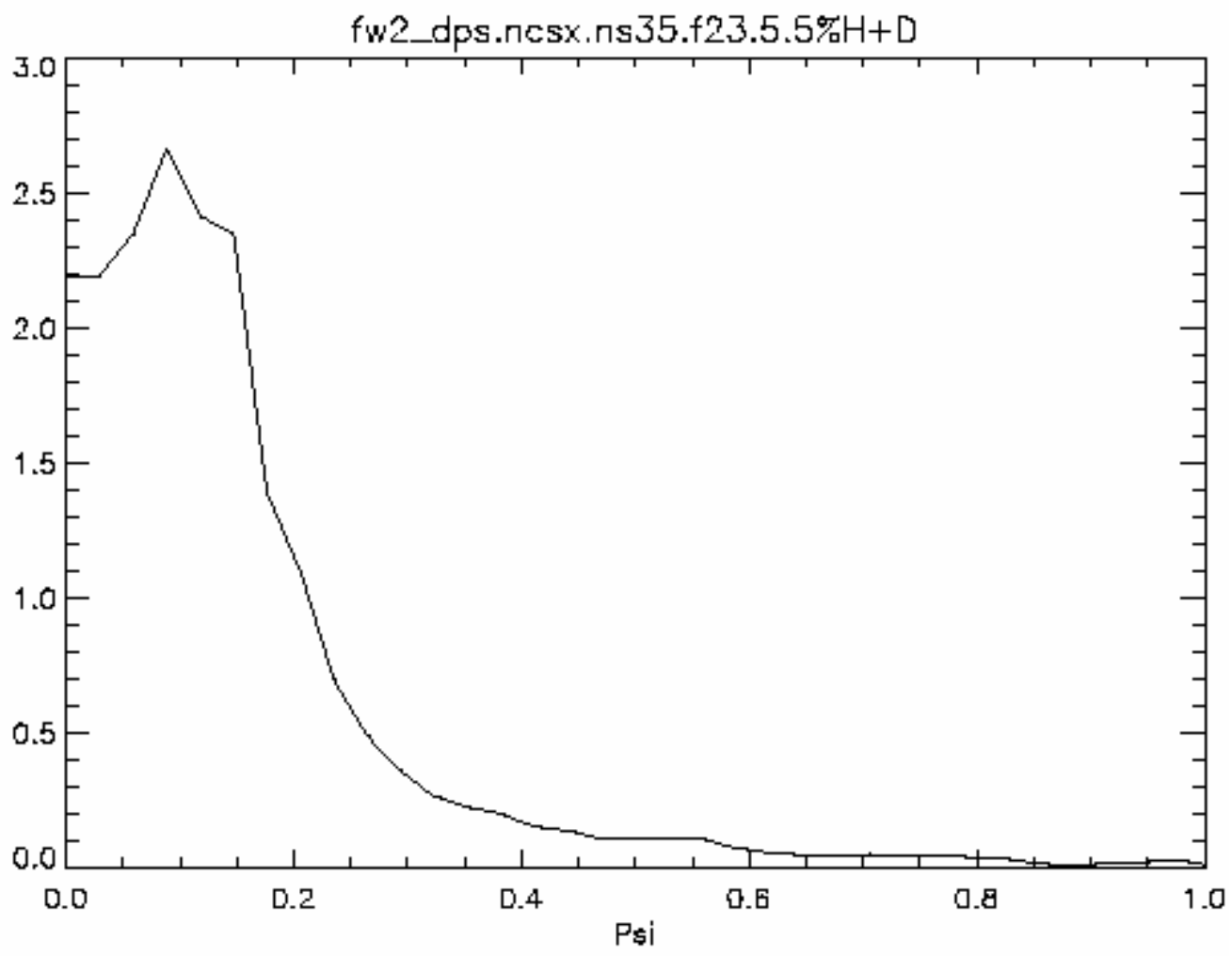

Fig.7.5 Radial power deposition to the protons in NCSX, 5\%H+D plasma, 


$$
F=23.5 \mathrm{MHz}
$$

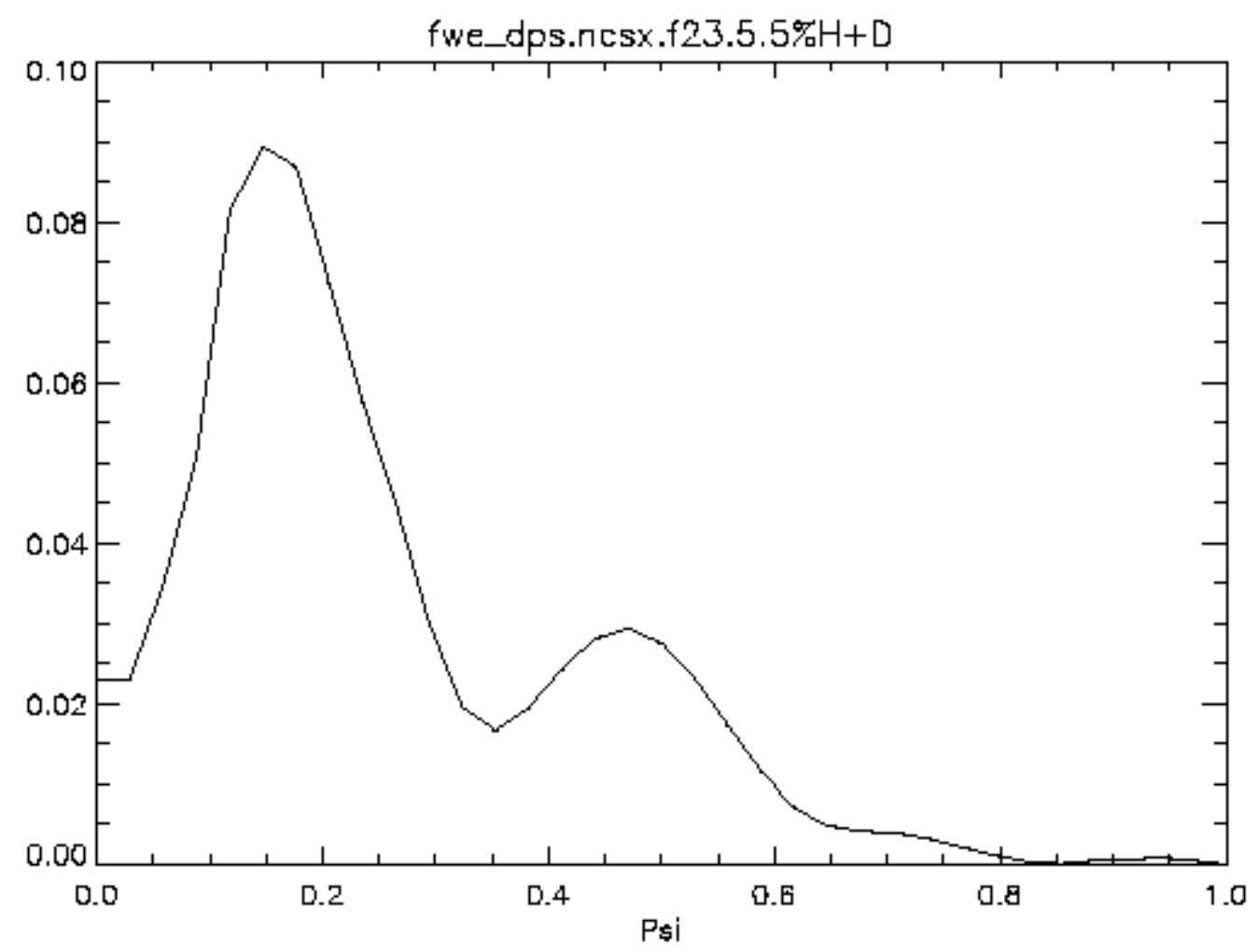

Fig.7.6 Radial power deposition to electrons in NCSX, 5\%

The minority scenario $\mathrm{D}(\mathrm{He} 3)$ is also interesting for NCSX due to possibility to easy control minority ions content and mode converted current drive at high He-3 content. The example of 3\% He-3 in deuterium plasma is presented by Figs.7.7, 7.8 showing radial power deposition profiles to He-3 ions and electrons. Generator frequency however must be about $15 \mathrm{MHz}$. Second He-3 harmonic at $30 \mathrm{MHz}$ may be interesting alternative at high beta expected in NCSX. 


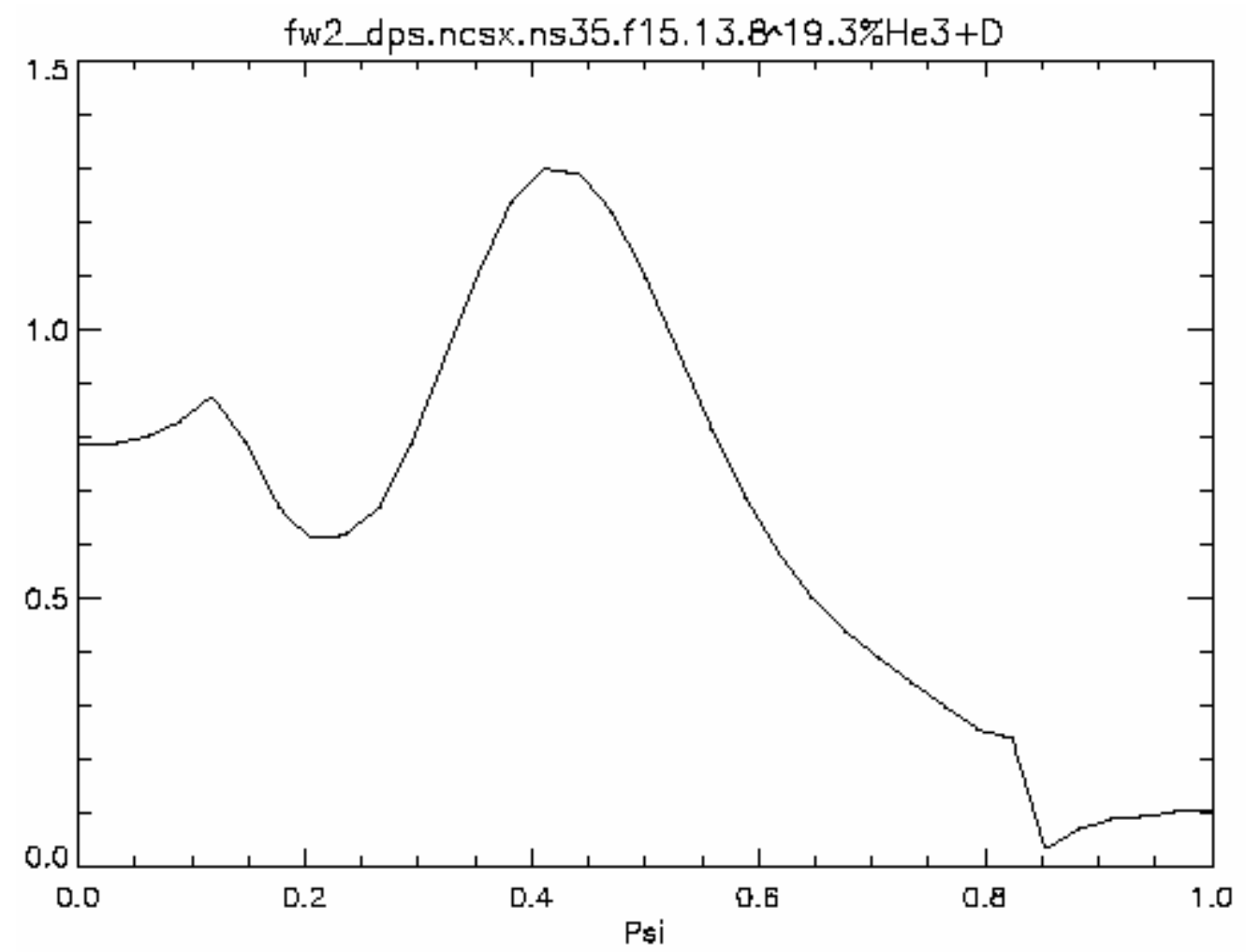

Fig.7.7 Radial power deposition to the protons in NCSX, 3\%He3+D plasma, $F=15.13 \mathrm{MHz}$ 


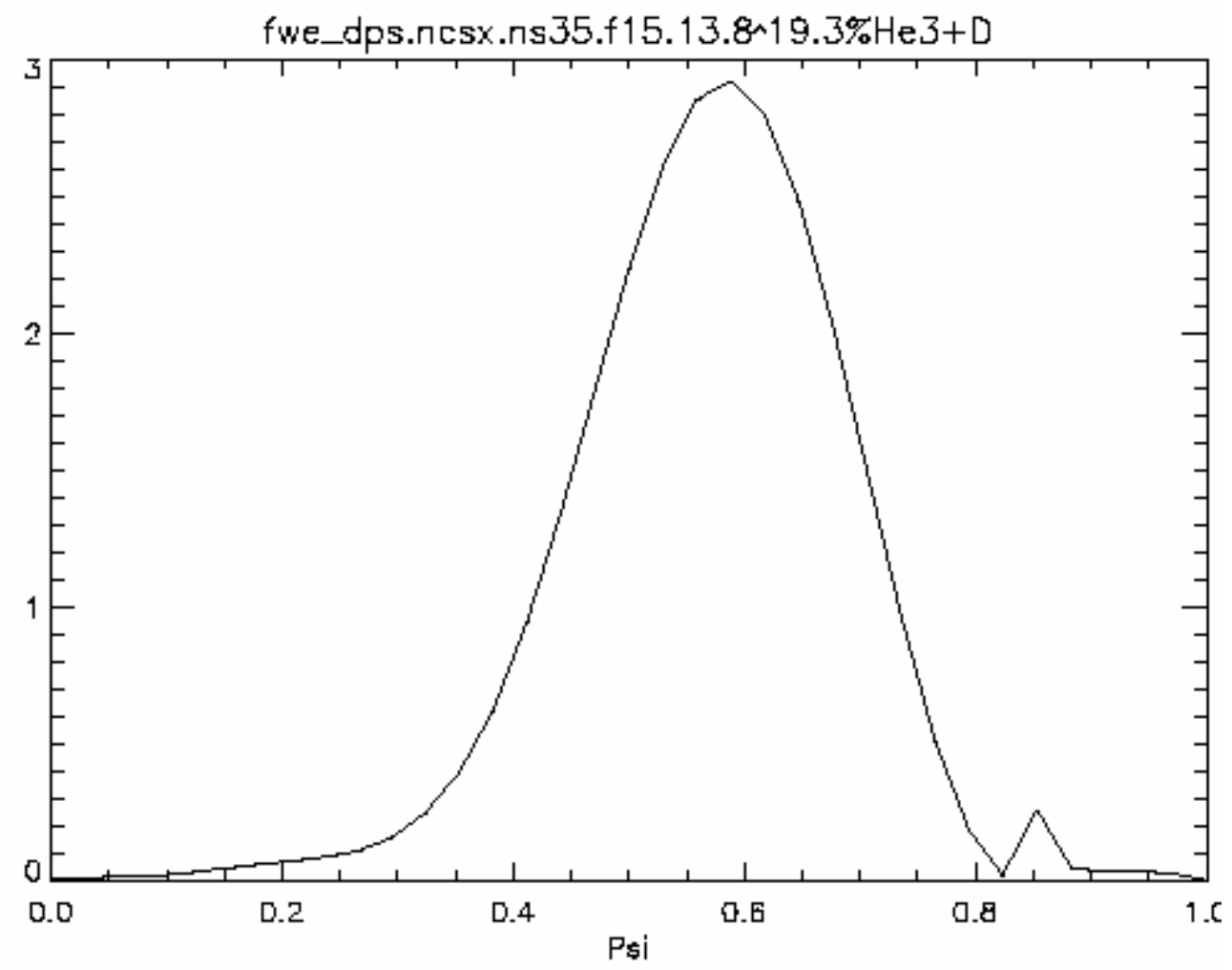

Fig.7.7 Radial power deposition to the electrons in NCSX, 3\%He3+D plasma, $F=15.13 \mathrm{MHz}$

Finally, second harmonic and minority ion heating ICRF scenarios in NCSX were found to be efficient with an outside or inside antennae. Light $\mathrm{D}(\mathrm{H}, \mathrm{He} 3)$ and heavy minority scenarios like $\mathrm{H}(\mathrm{D} / \mathrm{He} 4)$ are also efficient heating scenarios. Fundamental harmonic hydrogen heating scenario [2] must be included. Therefore a generator frequency range for NCSX of $15-50 \mathrm{MHz}$ is strongly recommended.

\section{Conclusions}

ICRF plasma heating has shown heating efficiencies comparable with NBI in large and middle-size stellarators like LHD and W7-AS for various heating schemes. On LHD the highest RF power coupled to the plasma was $2.7 \mathrm{MW}$ and the longest plasma sustainment was 32 minutes at $0.7 \mathrm{MW}$. The best heating performance has been observed with LFS and HFS antennae for minority heating schemes. Recent successful fundamental harmonic scenario at LHD at high plasma densities is an additional important possibility for ICRH in fusion machines and must be accounted at RF generator frequency band planning. 3D full wave ICRF PSTELION code reasonably models experiments and provides Guidance in optimizing heating scenarios for ion and electron heating in advanced stellarators like W7-X and NCSX. Code modeled in details many ICRF 
scenarios in the NCSX machine and found optimized frequency range $15-50$ $\mathrm{MHz}$ for RF generator for that machine. Steady state ICRF heating is a good candidate for a stellarator reactor.

Acknowlegments. Author appreciate help of Drs. F.Wesner, D.Hartman, A.Fukuyama, T.Watari, D.Monticello in several years work joint work on ICRF heating in stellarators. Author thanks M.Zarnstorff for interest of ICRF scenarios to NCSX and its equilibrium data base providing.

Invaluable were elucidating discussions with Profs. M.Brambilla, J.Nuhrenberg and R.Wilhelm. Author also thanks for permanent help of Drs I.Weidl (RZG Germany) and DeBoni (LBL US) on parallel super computers problems.

Work supported by the U.S. Department of Energy under Grant DE-FG02-02ER54667

\section{Referencies}

1) T.Watari, et al., Nuclear Fusion 41 (2001) 325-332

2) T.Mutoh, et al., NIFS-741 (IAEA 2002 (Lyon) Conf. on Contr. Fusion,CN94/EX)

2a) Achievement of the World Record in the Long-Pulse Experiment for High-Temperature Plasma Sustainment Using the Large Helical Device (LHD) Uniquely Developed in Japan, NIFS press report 17 December 2004

3) V.Vdovin, T.Watari, A.Fukuyama, NIFS-469 (1996)

4) D.Hartmann, et al., 27th EPS Conf. Contr. Fusion and Plasma Physics, Budapest, 2000, 24B.

5) G.Neilson, et al., PPPL-3676 (2002

6) V.Vdovin et all, $30^{\text {th }}$ EPS Conf. Contr. Fusion and Plasma Physics, St Petersburg, 2003, O-4, 3B 


\title{
Study of HFFW heating and current drive in the DIII-D tokamak QDB scenario
}

\author{
Vdovin V.L. \\ RRC Kurchatov Institute, Nuclear Fusion Institute \\ Moscow, Russia \\ Work supported by \\ the U.S. Department of Energy under Grant DE-FG02-02ER54667
}

\begin{abstract}
Initial modelling of IC heating and current drive in the ITER-like steady state Scenario 4 for the Quiescent Double Barrier high elongation and triangularity plasma parameters of DIII-D [1] with newly developed PSTELION 3D ICRF full wave code was performed. The main goal was to maximize off-axis current drive (CD) in ICRF frequency range. The High Frequency Fast Wave (HFFW) current drive scenario at $\mathrm{f}=$ $120 \mathrm{MHz}\left(\omega \sim 8 \omega_{\mathrm{cD}}\right)$ was shown be capable to create Far Outside non inductive driven current profile in DIII-D, urgently needed for the ITER steady state Scenario [2]. Dominant proton heating scenario in deuterium plasma with the hydrogen $6-60 \%$ addition is also possible in DIII-D with a power deposition to the ions in a broad middle of minor radius plasma region thus providing conditions for high energy protons tail population creation. This IC

Heating scenario may be an alternative to the NB counter injection method for the QDB regime creation due to radial electrical fields production by unconfined energetic protons effects. Combined operation of NBI and IC methods may be also possible in QDB regimes.
\end{abstract}

\section{High Frequency Fast Wave current drive in DIII-D reversed shear scenario}

Recently important results on HFFW electron H/CD were obtained at NSTX (EPS 28, Madeira and Lyon IAEA conf. [ 5 ]): efficient electron heating, driven current up to 0.1 MA, accompanied by internal inductance decrease. This scenario was also explored in previous DIII-D ICRF experiments at high ion cyclotron harmonics [3]. This scenario in ITER (200-300 MHz, SS sources $1 \mathrm{MW} /$ tube at $200 \mathrm{MHz}$ are available ones [ 4 ]) can provide RF driven current at the MIDDLE of plasma minor radius with efficiencies as high as 0.55 in usual units.

This is especially important because at IAEA Sorrento conference (2000) JT-60U reported NNB CD experiments $(\sim 400 \mathrm{keV})$ in conditions modelling the ITER $\left(\mathrm{V}_{\text {beam }} \sim\right.$ $V_{\text {Alfven }}$ ) with very bad results: instabilities (waited from the theory) have appeared and expelled to the walls energetic ions before their slow down (these results were repeated in following ITER meetings of our group). This information became even more worser with recent off-axis ASDEX NB CD experiments (Hobirk's paper [6] at EPS30, St- 
Petersburg, July 2003) which demonstrated NO any change in driven current PROFILE (JT-60U previously also reported similar results).

In such situation HFFW CD may substitute NB in ITER creating driven current at HALF of plasma minor radius (goal of NNB). Experimental HFFW activity on DIII-D tokamak is extremely important one to investigate this ITER-like scenario.

The main plasma and machine parameters used in the modelling are close to the present DIII-D the Quiescent Double Barrier (QDB) [ 1 ] and shown in Table 1:

\section{Table 1}

$\begin{array}{ll}\text { Major radius, } \mathrm{R} & 1.70 \mathrm{~m} \\ \text { Minor radius, a } & 0.60 \mathrm{~m} \\ \text { Plasma elongation, } \kappa & 1.8 \\ \text { Plasma triangularity, } \delta & 0.8 \\ \text { Nominal plasma current, } \mathrm{I}_{\mathrm{p}} & 1.3 \mathrm{MA} \\ \text { Toroidal field, } \mathrm{B}_{0} & 2.0 \\ \text { MHD axis safety factor, } \mathrm{q}_{\mathrm{o}} & 2.1 \\ \text { MHD safety factor, } \mathrm{q}_{\psi} 95 & 3.2\end{array}$

Table 2 sums the values for key plasma parameters in the considered DIII_D QDB shallow reversed shear discharge. The plasma parameters, presented in the Table, follow to the [1].

Table 2

\section{Plasma parameters of representative DIII-D QDB scenario}

Central deuterium temperature

Central hydrogen temperature

Central electron temperature

Temperature exponent

Central electron density
$\mathrm{T}_{\mathrm{D} 0}$

$\mathrm{T}_{\mathrm{H} 0}$

$\mathrm{T}_{\mathrm{e} 0}$

$\alpha_{\mathrm{T}}$

$\mathrm{n}_{\mathrm{e} 0}$
$15.2 \mathrm{keV}$

$15.2 \mathrm{keV}$

$5.4 \mathrm{keV}$

2

$6.27 \times 10^{19} \mathrm{~m}^{-3}$ 
Density exponent

Volume averaged density

Effective $\mathrm{Z}$ $\alpha_{\mathrm{n}}$

$$
<\mathrm{n}_{\mathrm{e}}>
$$

$\mathrm{Z}_{\text {eff }}$

RF power
0.15

$5.9 \times 10^{19} \mathrm{~m}^{-3}$

2.17

We modelled the ICRF ion heating/CD with tokamak 2D version of 3D full wave ICRF PSTELION code. This code version self consistently accounts for mode conversion effects and treats more accurately electron absorption accounting for finite electron Larmor radius effects. Magnetic configuration and input metric to the code comes from self consistent symmetrical ITER 2D calculations with equilibrium VMEC code [ 3 ].

Power deposition of RF FW power to the electrons in outer plasma region significantly increases at the frequency $120 \mathrm{MHz}$ (toroidal mode number $\mathrm{N}=10$ ) when second and third hydrogen harmonic resonances move behind of a plasma centre. Flux averaged radial power deposition profile to the electrons is shown at Fig.1. Electrons absorb 93.8\% of RF power leading to the global CD efficiency gamma $0.0346 \mathrm{~A} / \mathrm{W} / \mathrm{m}^{2}$. Deuterium third and forth harmonics absorb only $0.72 \%$ of RF power in this scenario.

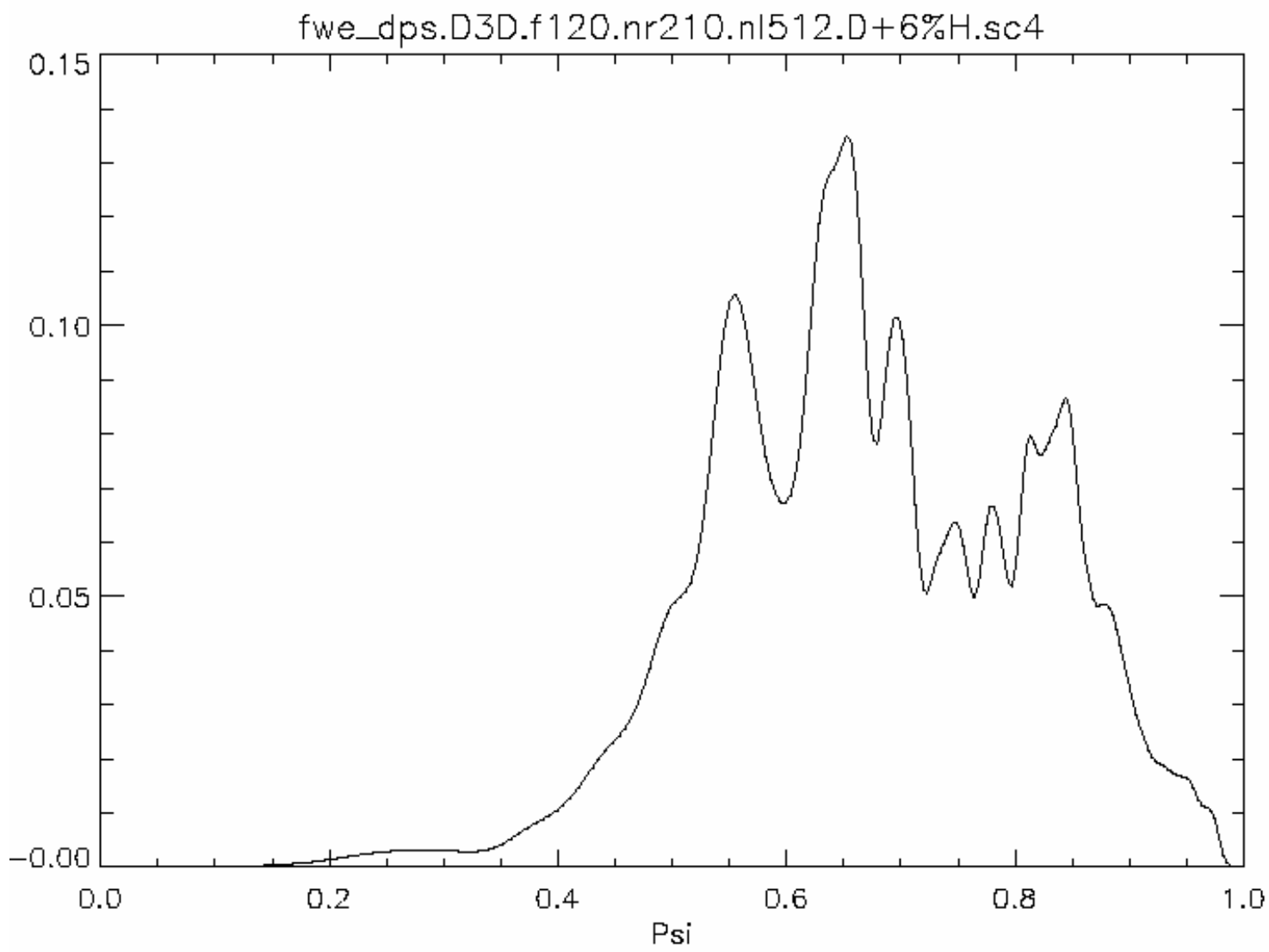


Fig.1 Radial power deposition to the electrons at frequency $120 \mathrm{MHz}$ versus $\operatorname{sqrt}(\rho)\left(\rho=\sqrt{\Phi / \Phi_{\text {sep }}}\right)$. Outside DIII-D antenna, $\mathrm{N}=10$. The $94 \%$ of RF power is absorbed by electrons.

The driven current profile is displayed in Fig.2. The current profile, being far off-axis, is very close to the profile needed to ITER SS scenario with shallow reversed shear [3].

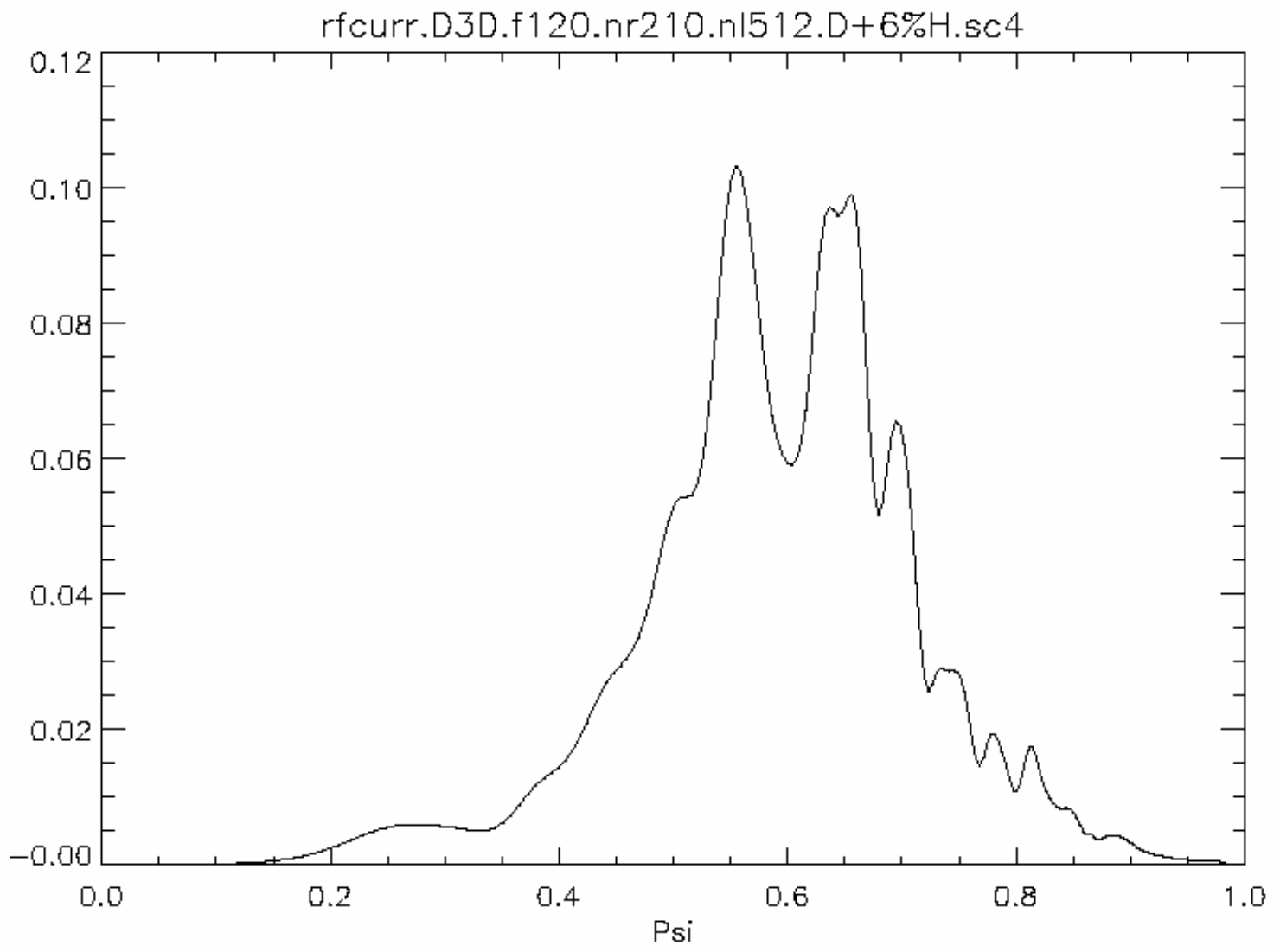

Fig.2 The RF driven current radial profile in DIII-D due to electron absorption at frequency $120 \mathrm{MHz}$ over $\operatorname{sqrt}(\rho)$. Outside antenna, $\mathrm{N}=10$. The $\mathrm{CD}$ efficiency is 0.0346 $\mathrm{A} / \mathrm{W} / \mathrm{m} 2$

\section{Ion heating at second hydrogen harmonic}

This is classical second harmonic scenario previously proposed for tokamaks and reactors. In our DIII-D modeling of QDB scenario In this QDB D+H scenario at frequency $60 \mathrm{MHz}$ and with Maxwellian distribution hydrogen absorbs up to $85 \%$ of incoming RF power in broad range of protons concentration. Presently we did not account to nonthermal deformation of protons' distribution function which may be important one (and it is planned to be done in future modelling). The Fig. 3 displays the power deposition profile to $6 \%$ protons in deuterium plasma at main antenna spectrum lob $<\mathrm{N}>=10$. The protons absorb $85.8 \%$ of RF power, electrons $-13.49 \%$. 


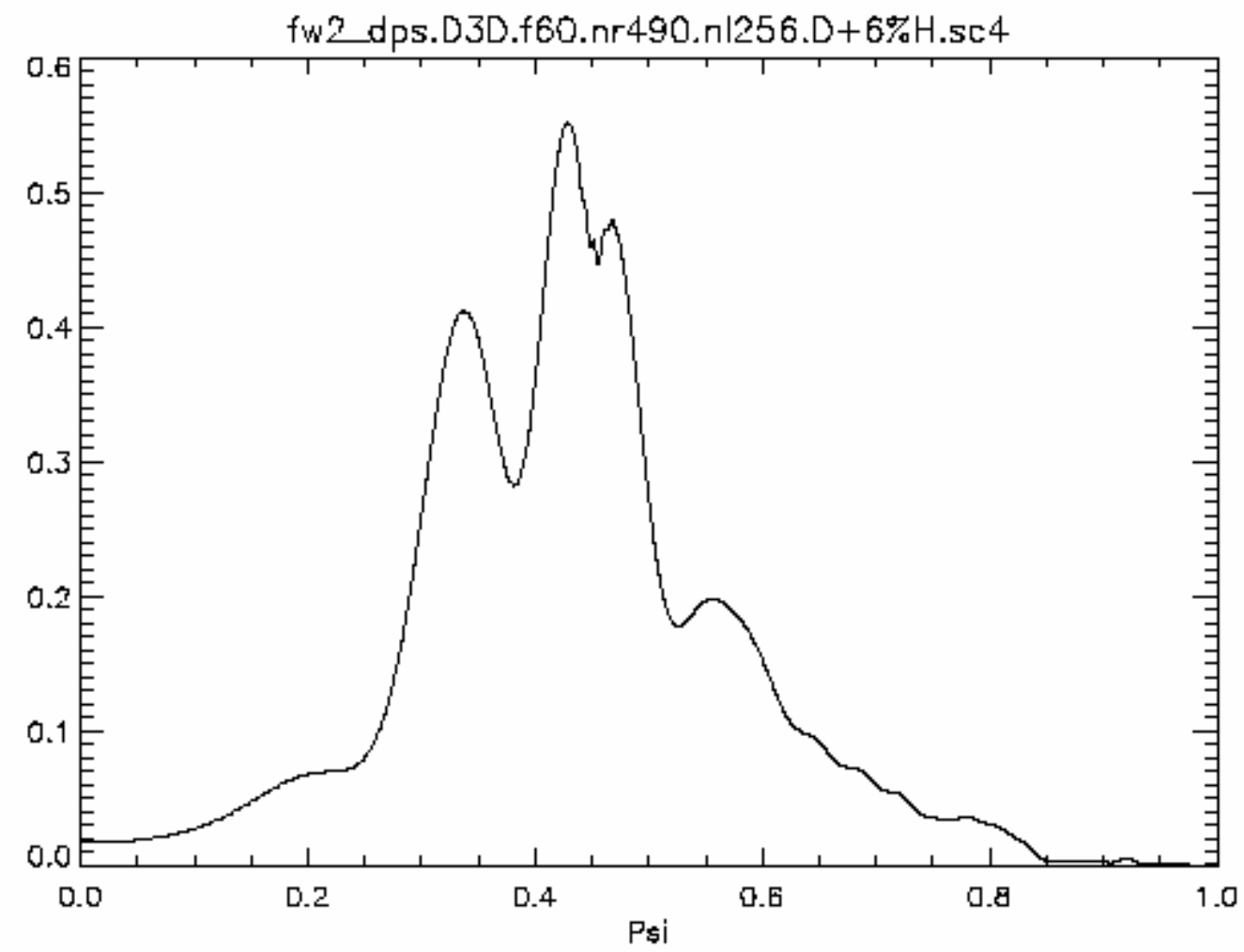

Fig.3 Radial power deposition to the protons at frequency $60 \mathrm{MHz}$ versus $\operatorname{Sqrt}(\rho)$. Outside DIII-D antenna, $\mathrm{N}=10$. The $85.8 \%$ of RF power is absorbed by electrons.

The power deposition to the electrons is given in Fig.4. 


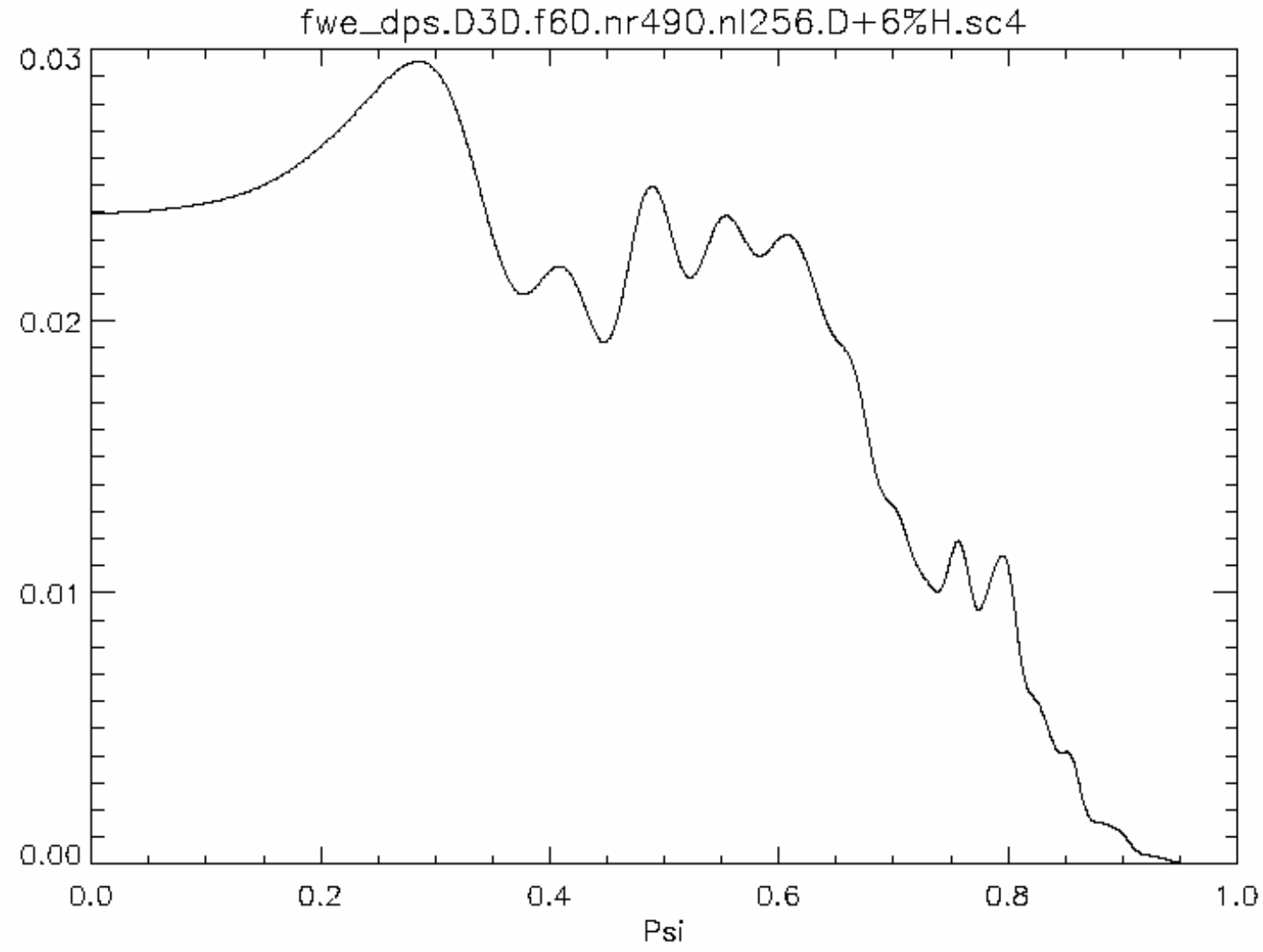

Fig.4 Radial power deposition to the electrons at frequency $60 \mathrm{MHz}$ versus $\operatorname{sqrt}(\rho)$. Plasma ion composition is $\mathrm{D}+6 \% \mathrm{H}$. Outside DIII-D antenna, $\mathrm{N}=10$. The $13.5 \%$ of RF power is absorbed by electrons.

Driven current radial profile, being centrally peaked at this frequency, is displayed by Fig.5. The CD efficiency is $0.007 \mathrm{~A} / \mathrm{W} / \mathrm{m} 2$. 


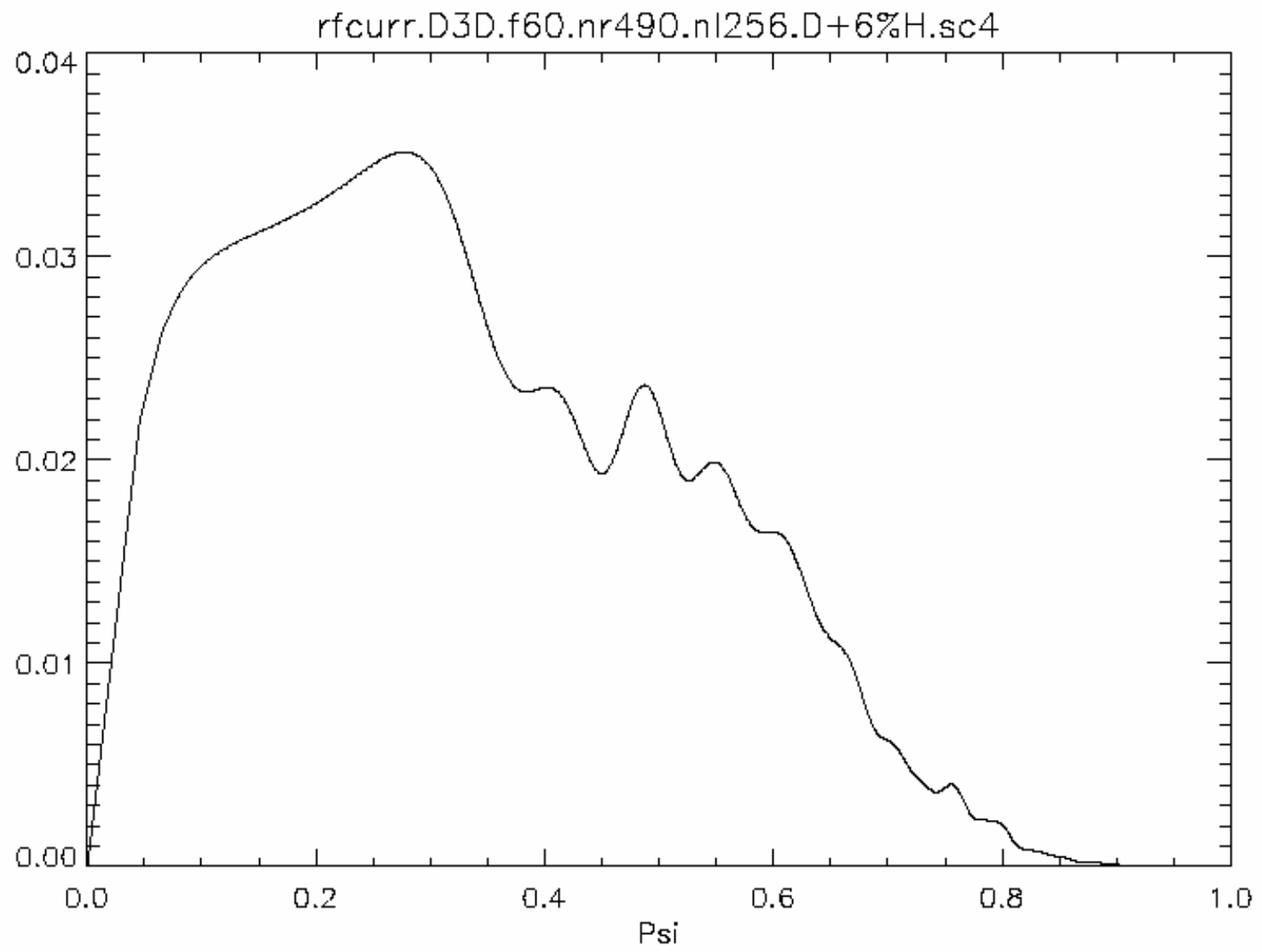

Fig.5 The RF driven current radial profile in DIII-D due to electron absorption at frequency $60 \mathrm{MHz}$ over sqrt $(\rho)$. Outside antenna, $\mathrm{N}=10$. The $\mathrm{CD}$ efficiency is 0.007 $\mathrm{A} / \mathrm{W} / \mathrm{m} 2$

\section{Conclusions}

Initial modelling of IC heating and current drive in the ITER-like steady state Scenario 4 for the Quiescent Double Barrier high elongation and triangularity plasma parameters of DIII-D with newly developed PSTELION 3D ICRF full wave code was performed. The main goal was to maximize off-axis current drive (CD) in ICRF frequency range. The High Frequency Fast Wave current drive scenario at $\mathrm{f}=120 \mathrm{MHz}$ $\left(\omega \sim 8 \omega_{\mathrm{cD}}\right)$ was shown be capable to create Far Outside non inductive driven current profile in DIII-D, urgently needed for proving advanced ITER steady state Scenario. Dominant proton heating scenario in deuterium plasma with the hydrogen $6-60 \%$ addition is also possible in DIII-D with a power deposition to the ions in a broad middle of minor radius plasma region thus providing conditions for high energy protons tail population creation. This IC

heating scenario may be an alternative to the NB counter injection method for the QDB regime creation due to radial electrical fields production by unconfined energetic protons effects. Combined operation of NBI and IC methods may be also possible in QDB regimes. 
Thus ICRF method has good prospects in experiments on DIII-D to support advanced ITER scenarios.

Acknowledgements Advices and positive support to HFFW scenarios of Dr. R.Prater is strongly appreciated.

\section{Referencies}

1. Doyle D,J. and DIII-D team THE QUIESCENT DOUBLE-BARRIER REGIME IN THE DIII-D TOKAMAK

Invited talk at $28^{\text {th }}$ Eur. Conf. on Contr. Fusion and Plasma Physics

2. Technical Basis for the ITER Final Design Report, JCT Garching-Naka, ITER scenarios 2003 from JCT

2a. Plasma of ITER Scenario 4, TASK 2003 for ICRF modeling by Y.Gribov, ITER JCT Garching-Naka, Japan

3. Prater R. et all, FAST WAVE HEATING AND CURRENT DRIVE IN DIII-D DISCHARGES WITH NEGATIVE CENTRAL SHEAR, IAEA Conference on Plasma Physics and Controlled Nuclear Research, October 7-11, 1996, Montreal,

4. Thomson Tube Electronics Design Study "ICRH radio frequency generators", report in achievement of the tasks D.350.6 \& D.350.7 ordered under contract \#93-851/EH, December 1999

5. PPPL's EPS 28 (Madeira, 2001), Hosea J. et all, and IAEA 2002 (Lyon) conferences papers

6. Hobirk J. Off axis neutral beam current drive experiments on Asdex-U and JT60-U Oral $-4,1 B, 30^{\text {th }}$ Eur. Conf. on Contr. Fusion and Plasma Physics, St Petersburg, July $7-11,2003$ 


\title{
ICRF benchmarking modelling of ITER scenario \#2
}

\author{
Vdovin V.L.
}

RRC Kurchatov Institute, Nuclear Fusion Institute

Symposium Celebrating the 70th Birthday of Arnold H. Kritz Integrated modeling of magnetically confined plasmas PPPL, NJ US, 19-20 July 2005

ITER Physics Tasks meeting Moscow , June 8-9, 2005

7th Steady State Operation ITPA Topical Group meeting Como, Italy, May 4-6, 2005

Work supported by the U.S. Department of Energy under Grant DE-FG02-02ER54667 


\section{OUTLINE}

- Brief review of PSTELION 3D full ICRF code

- Benchmarking modelling of scenario \#2

- Tritium second harmonic 


\section{PSTELION full wave 3D ICRF code}

- Motivation

Mainly ICRF heating

- scenarios development

- optimised antennae

Also

- Low frequency instabilities (Alfven eigenmodes)

- Diagnostic (Ion Cyclotron Emission)

- Features

- Configuration: 3D, 2D (tokamaks included)

- Hot plasma model: Ion Bernstein and Kinetic Alfven Waves, FLR effects - through second order expansion

- Numerical method:

- Finite differences, Modes expansion

- Magnetic flux coordinates

- Plasma equilibrium - VMEC (ORNL) code 


\section{Full wave formulation and Spectral expansion used in PSTELION}

- Solve Maxwell-Vlasov equations for bounded plasma with fixed frequency and linear plasma response in a spectral -central difference method using the 3D PSTELION EM field solver

$$
\begin{aligned}
& \nabla \times \nabla \times \mathrm{E}=\frac{\omega^{2}}{c^{2}}\left[\mathrm{E}+\frac{4 \pi i}{\omega}\left(\mathrm{J}^{p}+\mathrm{J}^{A N T}\right)\right] \\
& J_{P}=\hat{\sigma} E
\end{aligned}
$$

Sigma being evaluated at ion FLR $\operatorname{limit} \frac{1}{2}\left(k_{\perp} \rho_{t}\right)^{2}<1$

$$
E(r)=\sum_{m, n} E(\psi) \exp (\operatorname{im} \theta+\operatorname{in} \varphi)
$$




\section{Parallel implementation of PSTELION code}

- The code uses "Progonka" Algorithm (Gaussian inversion specialized for three block diagonal system) for parallel processors computer option, distributing matrices across processors, and uses the ScaLapack library and message passing interface (MPI) for parallel matrix operations. 


\section{Resolution requirements for the code \\ in ITER scenario \#2 simulations}

- Required number of radial elements $\left(\mathrm{N}_{\psi}\right)$ and poloidal modes $\left(\mathrm{N}_{\mathrm{m}}\right)$ are determined by the shortest wavelength in system:

- For the mode converted IBW:

$$
\mathrm{k}_{\perp} \rho_{\mathrm{i}} \approx 1 \text { and }\left.\lambda_{\perp}\right|_{\mathrm{ibw}} \approx 3 \mathrm{~cm}
$$

- For the mode converted SW for ITER typically

$$
\begin{aligned}
& \omega / \mathrm{k}_{/ /}<\mathrm{V}_{\mathrm{Te}} \text { and } \varepsilon_{33} \approx\left(\omega_{\mathrm{pe}} / \mathrm{k}_{/ /} \mathrm{V}_{\mathrm{Te}}\right)^{2} \\
& \mathrm{k}_{\perp} \approx(\omega / \mathrm{c})\left(\varepsilon_{33}\right)^{1 / 2}=\omega \omega_{\mathrm{pe}} / \mathrm{ck}_{/ /} \mathrm{V}_{\mathrm{Te}}
\end{aligned}
$$

Taking $\mathrm{k}_{\perp} \approx 1 / \rho_{\mathrm{i}}$ one finds: $\left.\lambda_{\perp}\right|_{\mathrm{sw}} \approx 3 \mathrm{~cm}$ occurs at $\mathrm{k}_{/ /}=2 / 7 \mathrm{~cm}^{-1}$ In ITER above and lower of equatorial plane $k_{/ /}$quickly rises and SW (named as Kinetic Alfven Waves - KAW) dominate 


\section{Resolution requirements for the code in ITER simulations (Ctd)}

- Radial resolution for converted SW and IBW waves is determined by:

$$
\mathrm{N}_{\psi} \gg\left(\mathrm{a} / \lambda_{\psi}\right) \text { where }\left.\lambda_{\psi}\right|_{\text {converted }} \text { So we take } \mathrm{N}_{\psi} \geq 470
$$

- Poloidal mode resolution is determined by

$2 \pi / \lambda_{\text {converted }} \approx \mathrm{m} / \mathrm{r}$, at $\mathrm{r}=20 \mathrm{~cm}$ one has $\mathrm{m}=41$

so $\geq 128$ poloidal modes $(-64,64)$ look sufficient ones 


\section{Plasma geometry \& parameters}

- Major radius, $\mathrm{R}$

- Minor radius, a

- Plasma elongation, $\kappa$

- Plasma triangularity, $\delta$

- Nominal plasma current, Ip

- Toroidal field, B0

- (at $R=6.2 \mathrm{~m})$

- MHD safety factor, $q_{\psi 95}$
$6.20 \mathrm{~m}$

$2.0 \mathrm{~m}$

1.71

0.45

$15 \mathrm{MA}$

$5.3 \mathrm{~T}$

3.42 


\section{Plasma parameters of representative ITER scenario \#2}

- Central deuterium temperature

- Central tritium temperature (supported by 2D F-P code)

- Central electron temperature

- Separatrix temperatures

- Temperatures exponent, $\alpha_{T}$

- Alphas "temperature" exponent

- Central electron density

- Separatrix electron density

- Central deuterium/He4/Be temperatures

- Density exponent, $\alpha_{n}$

- Impurity fractions: $f_{H e}$, Alphas

- Effective Z

- RF power
$21.2 \mathrm{keV}$

$27.2 \mathrm{keV}$

$24.8 \mathrm{keV}$

$2.25 \mathrm{keV}$

2.0

0.0

$1.02 \times 10^{20} \mathrm{~m}^{-3}$

$0.6 \times 10^{20} \mathrm{~m}^{-3}$

$21.2 \mathrm{keV}$

0.15

$0.034,0.006$

1.71

$20 \mathrm{MW}$ 


\section{Benchmarking ITER ICRF parameters}

- Frequency

- Toroidal mode number, $\mathrm{N}$

- Antenna vertical displacement (down) poloidal LC-ant RF current slow down
$53 \mathrm{MHz}$

27

$0.6 \mathrm{~m}$

1.6 


\section{Fast Waves are well focussed}

$\operatorname{Re}($ E_plus) contours

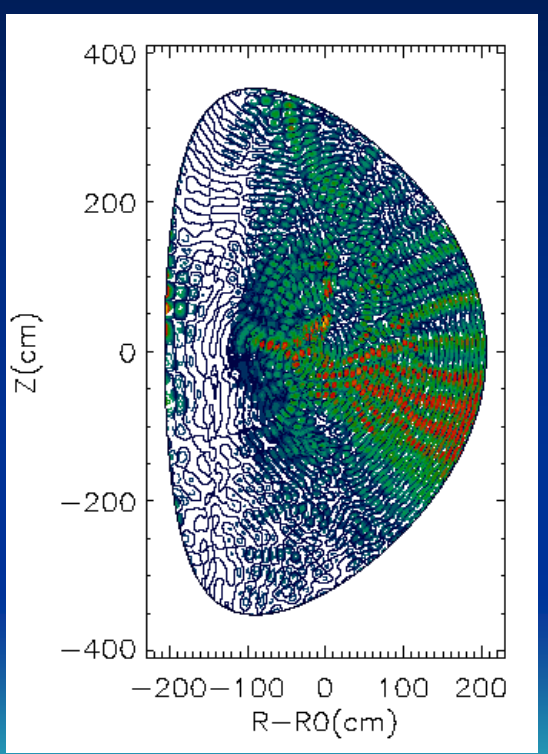




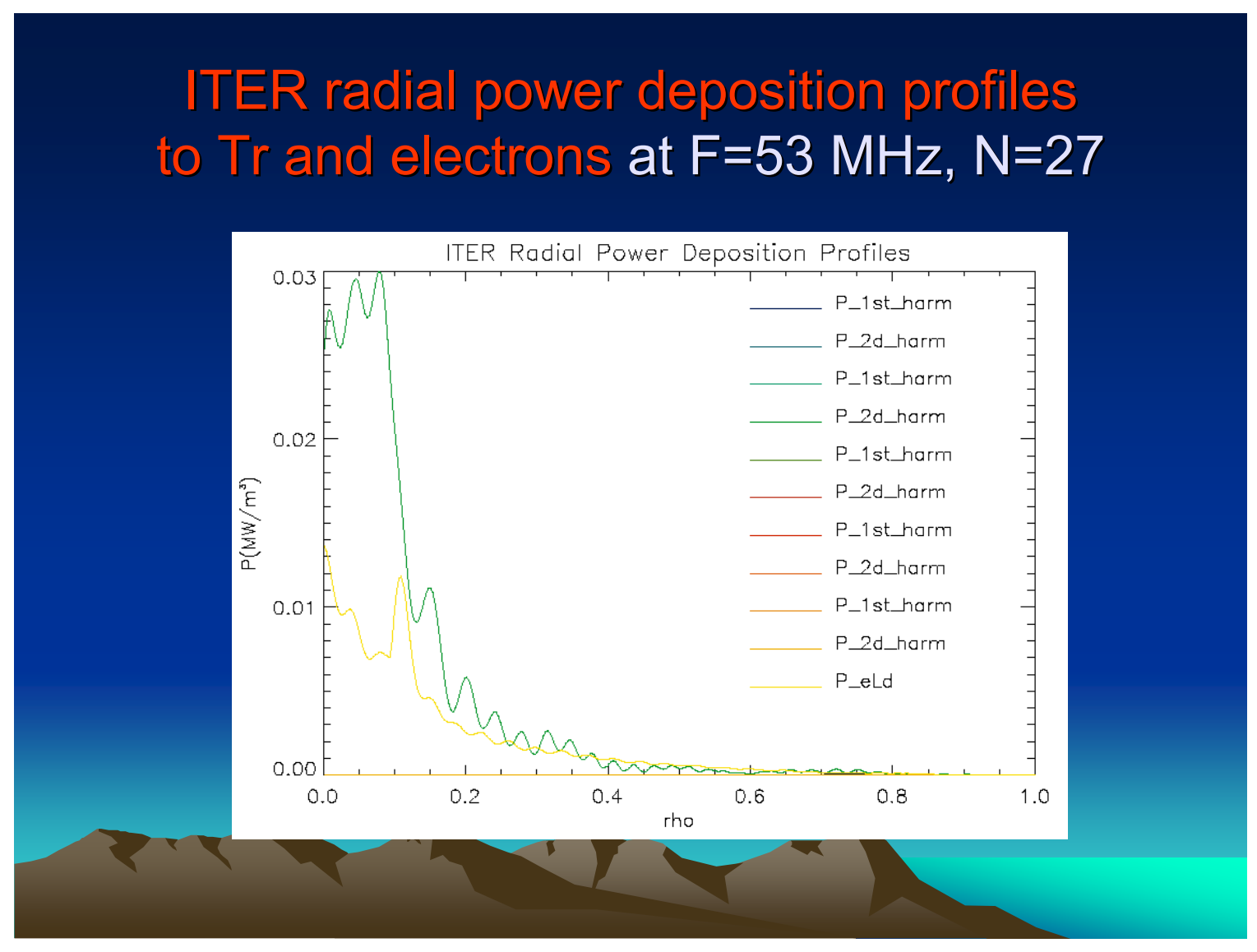




\section{ITER radial power deposition profiles at $\mathrm{F}=53 \mathrm{MHz}, \mathrm{N}=27$ and $2 \% \mathrm{Be}-9$}

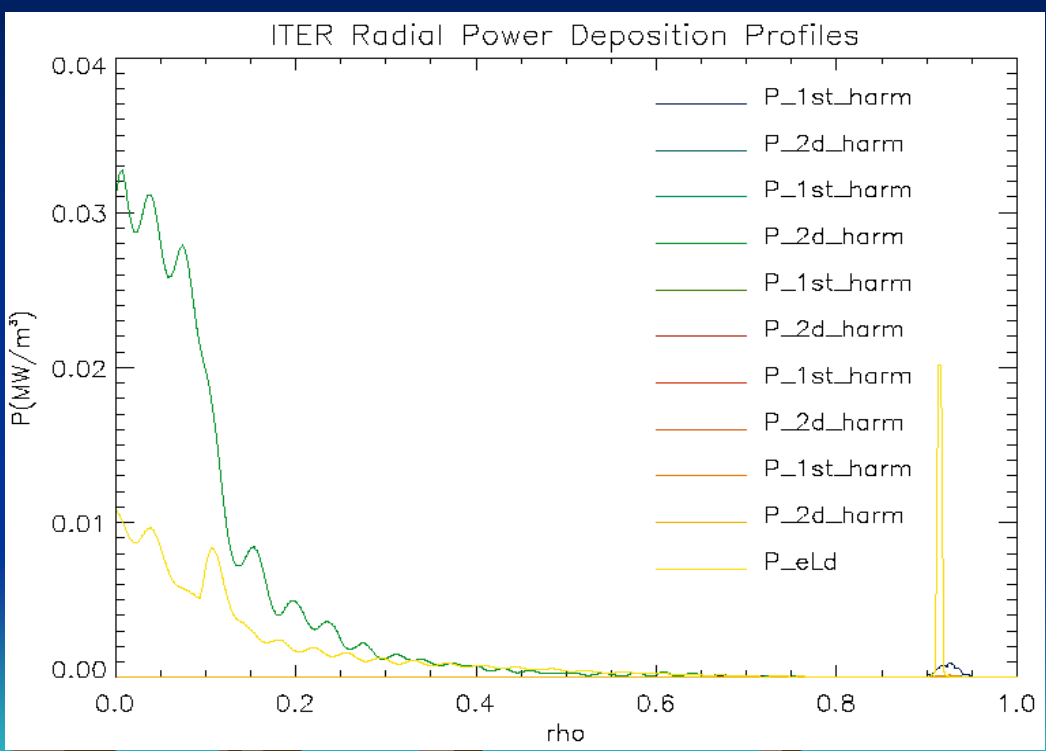




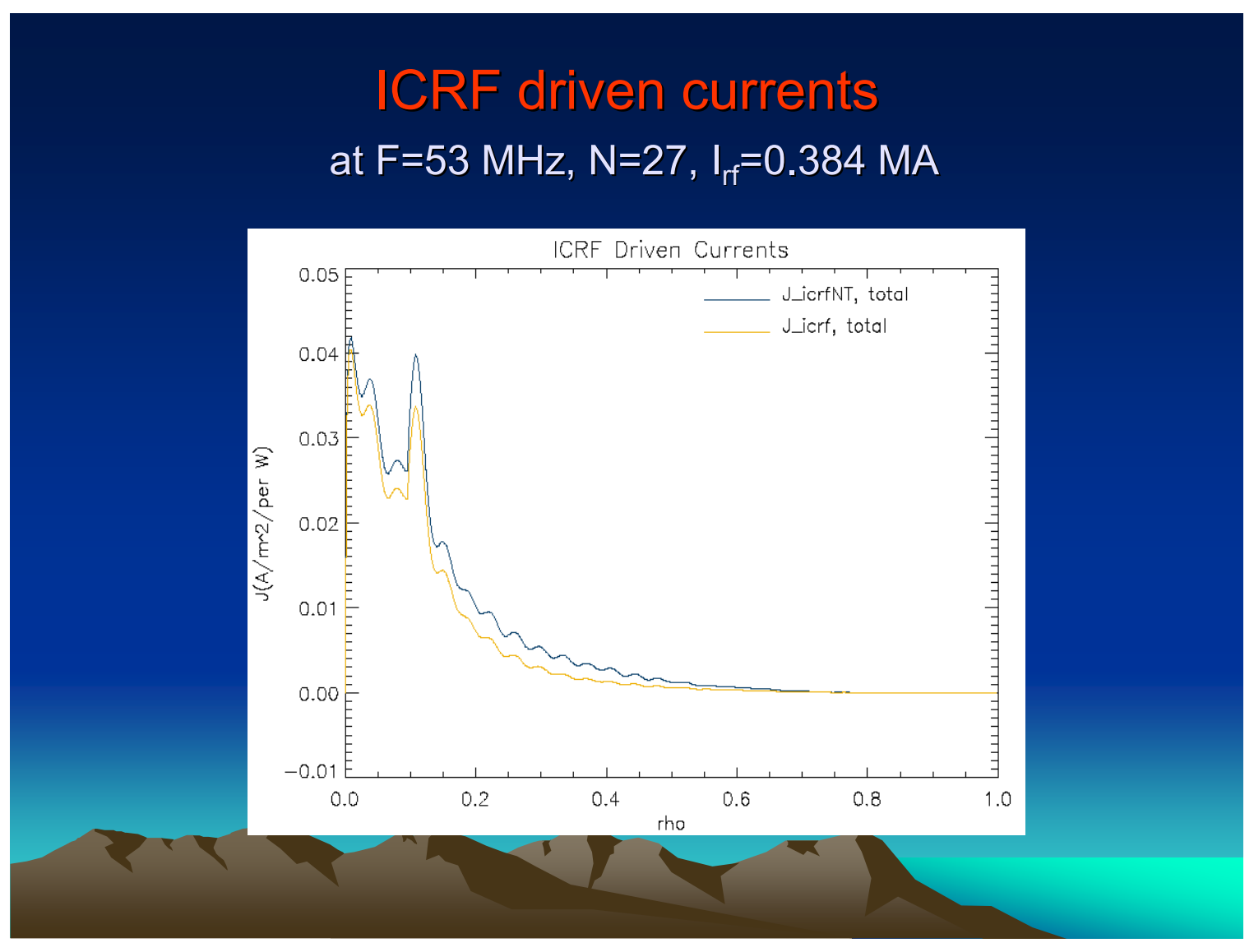




\section{Power deposition to Tritons at $\mathrm{F}=53 \mathrm{MHz}, \mathrm{N}=27$}

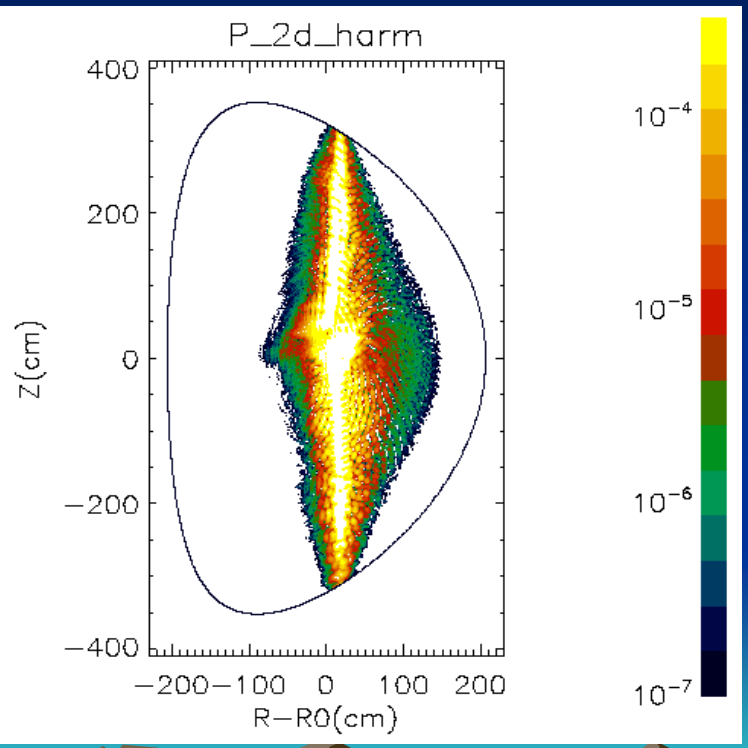




\section{Power deposition to Deuterons at $\mathrm{F}=53 \mathrm{MHz}, \mathrm{N}=27$}

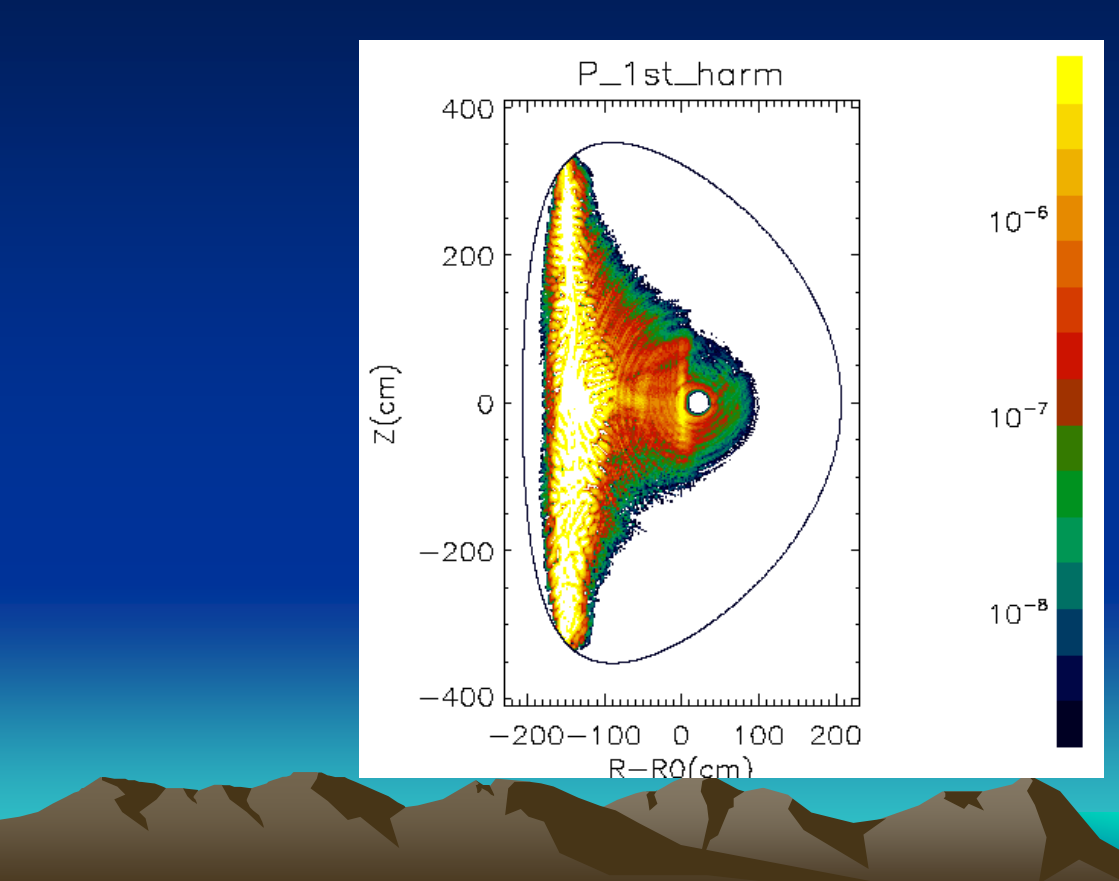




$$
01
$$




\section{Hot Alphas profile is narrow in ITER}

$$
\begin{aligned}
& \mathbf{N}_{D} N_{T}<\sigma V>=N_{\alpha} / \text { taU }_{\text {Spitzer }} \\
& <\sigma V>=C_{\alpha} T_{i}^{2} \\
& \operatorname{taU}_{\text {Spitzer }}=C_{s} T_{e}^{3 / 2} / N_{e} \\
& N_{\alpha}(s)=0.25 C_{\alpha} C_{s} N_{e}(s) T_{i}^{7 / 2}(\mathbf{s})
\end{aligned}
$$

- Very peaked radial profile of burning Alphas

This is slightly flattening due to banana orbits and transport effects, so we have taken moderate

$$
\mathbf{N}_{\alpha}(\mathbf{s}) \sim \mathbf{N}_{\mathrm{e}}(\mathbf{s})^{-3}(\mathbf{s})
$$


D 


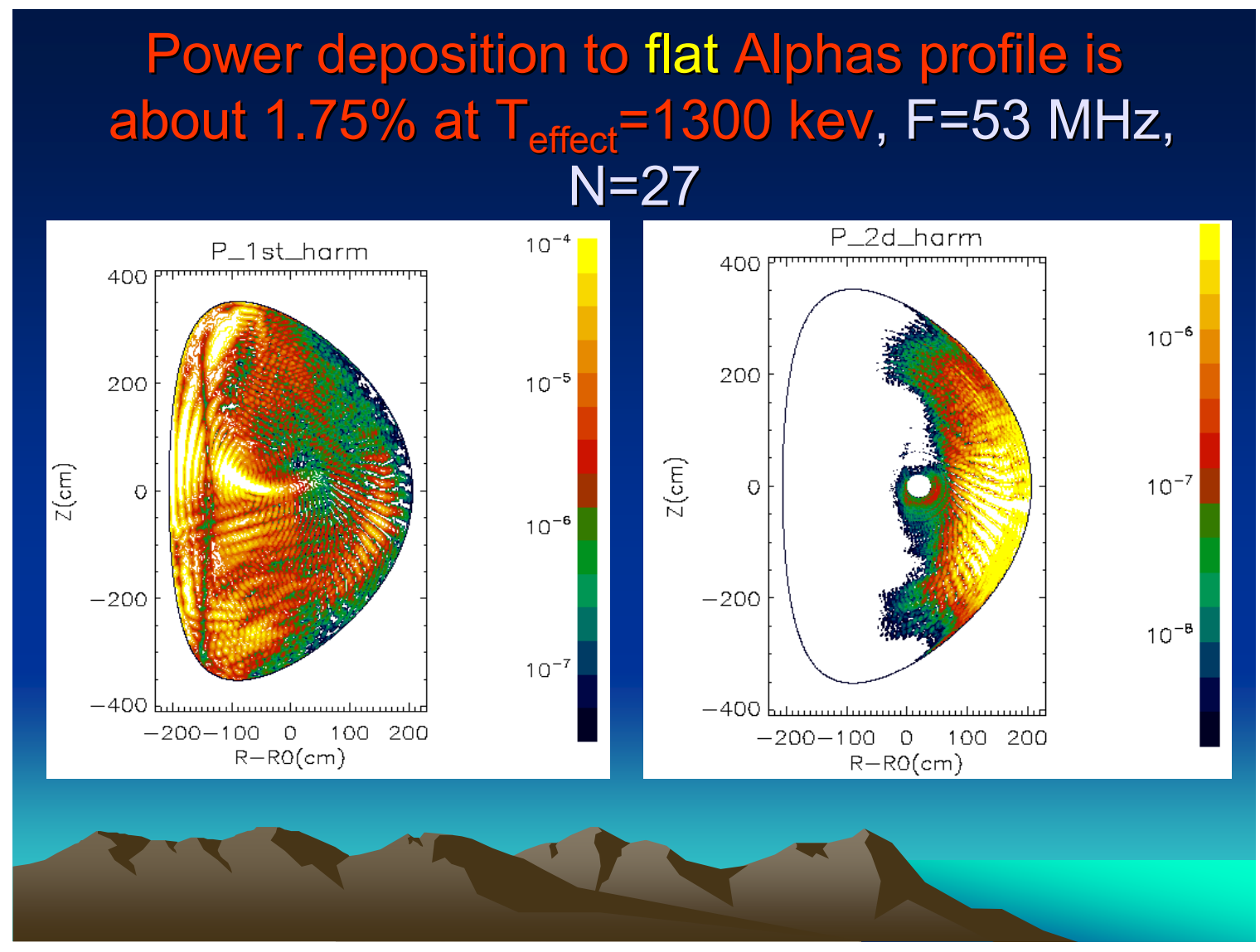




\section{Power partitions at $\mathrm{F}=53 \mathrm{MHz}, \mathrm{N}=27$}

- Power to Tritons

- Power to Deuterons

- Power to helium ash

- Power to Alphas

- Power to electrons power to elec-s by FW power to elec-s by SW

- Driven RF current
$58 \%$

$1.24 \%$

$0.15 \%$

$0.13 \%$

$40.49 \%$

$34.26 \%$

$6.22 \%$

$0.384 \mathrm{MA}$ 
ICRF Power balance in PSTELION, AORSA and TORIC

$$
\begin{aligned}
& \text { at } \mathrm{F}=53 \mathrm{MHz}, \mathrm{N}=27 \text { (COMO May } 2005 \text { ITPA meeting) } \\
& \text { PSTELION AORSA2D TORIC }
\end{aligned}
$$

$\mathrm{P}\left(2 \Omega_{\mathrm{T}}\right)$

$$
58 \% \quad 45 \% \quad 50 \%
$$

$\mathrm{P}$ (Electr)

$$
40.5 \% \quad 38 \% \quad 35 \%
$$

$P(D)$

$$
1.2 \% \quad-0.5 \% \quad 1.8 \%
$$

$\mathrm{P}$ (Alpha)

Flat Alphas: $1.75 \%$

Ash as "Alphas"
$\mathrm{P}(\mathrm{He})$
$0.15 \%$ 


\section{Power partitions at $\mathrm{F}=53 \mathrm{MHz}, \mathrm{N}=15$}

- Power to Tritons $\quad 76.5 \%$

- Power to Deuterons $\quad 1.08 \%$

- Power to helium ash $\quad 0.13 \%$

- Power to Alphas $\quad 0.13 \%$

- Power to electrons $\quad 22.17 \%$ power to elec-s by FW $\quad 16.1 \%$ power to elec-s by SW $\quad 6.07 \%$

- Driven RF current $\quad 0.108$ MA 


\section{Scenario $\mathrm{D}+\mathrm{T}+3 \% \mathrm{He}-3$ Power partitions at $\mathrm{F}=53 \mathrm{MHz}, \mathrm{N}=15$}

- Power to He-3

- Power to Tritons

- Power to Deuterons

- Power to helium ash

- Power to Alphas

- Power to electrons power to elec-s by FW power to elec-s by SW
$69.85 \%$

$11.01 \%$

$0.11 \%$

$0.02 \%$

$0.06 \%$

$18.96 \%$

$16.14 \%$

$2.83 \%$

- He-3 effective temperature (2D F-P code coupled with Wave code) 


\section{Conclusions}

- Up to $60 \%$ of RF power is absorbed by ions at second harmonic scenario at $\mathrm{F}=53 \mathrm{MHz}, \mathrm{N}=27$

- Ion absorption rises to $80 \%$ at $\mathrm{N}=15$ (non thermal q-l effects are included by 2D F-P)

- Rest of power is absorbed by electrons, mainly through Fast Wave absorption

- Inclusion of $2 \%$ Beryllium creates $\mathrm{D}^{-B^{9}}{ }^{9}$ hybrid resonance at inner plasma surface with accompanying numerical problems

- Scenario with $3 \% \mathrm{He}-3$ ions also provides dominant ion heating (up to $82 \%$ at $\mathrm{N}=15 \& \mathrm{~N}=27$ ) 